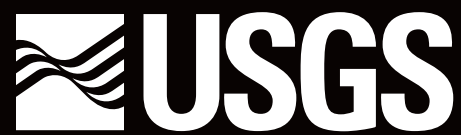

science for a changing world

Prepared in cooperation with the City of Columbia, Missouri, and the Missouri Department of Conservation

\title{
Water-Quality Data, 1999-2005, and Ground-Water Level Data, 2004-2005, for McBaine Bottoms, Including the Eagle Bluffs Conservation Area, Columbia, Missouri
}

Data Series 212 


\section{Water-Quality Data, 1999-2005, and Ground-Water Level Data, 2004-2005, for McBaine Bottoms, Including the Eagle Bluffs Conservation Area, Columbia, Missouri}

By Brenda J. Smith and Joseph M. Richards

Prepared in cooperation with the

City of Columbia, Missouri,

and the Missouri Department of Conservation

Data Series 212 


\title{
U.S. Department of the Interior DIRK KEMPTHORNE, SECRETARY
}

\author{
U.S. Geological Survey \\ Mark D. Myers, Director
}

\section{U.S. Geological Survey, Reston, Virginia: 2006}

For sale by U.S. Geological Survey, Information Services

Box 25286, Denver Federal Center

Denver, CO 80225

For more information about the USGS and its products:

Telephone: 1-888-ASK-USGS

World Wide Web: http://www.usgs.gov/

Any use of trade, product, or firm names in this publication is for descriptive purposes only and does not imply endorsement by the U.S. Government.

Although this report is in the public domain, permission must be secured from the individual copyright owners to reproduce any copyrighted materials contained within this report. 


\section{CONTENTS}

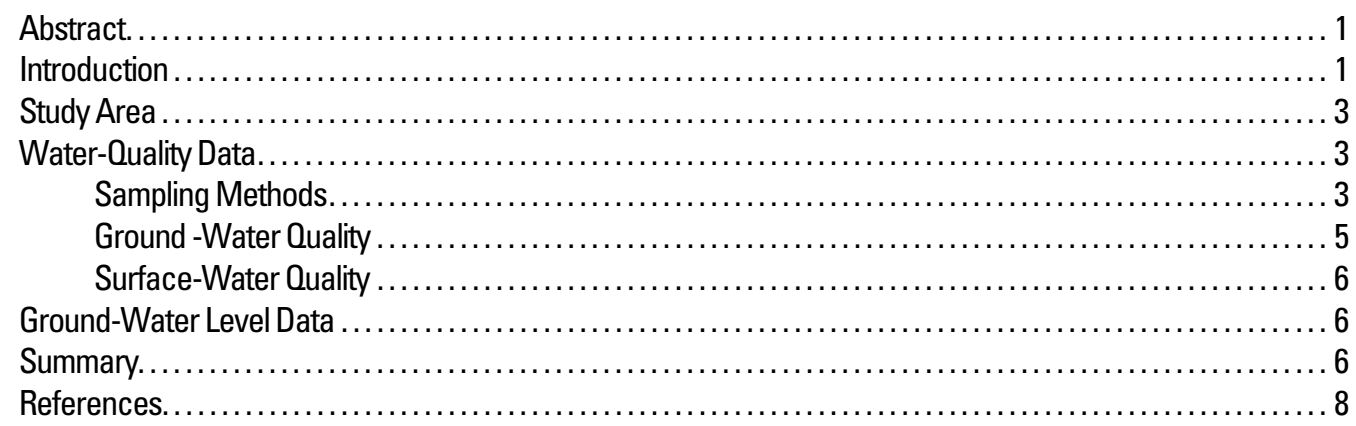

\section{Figures}

\section{1-3. Maps showing:}

1. Location of McBaine Bottoms, Columbia, Missouri ...............................

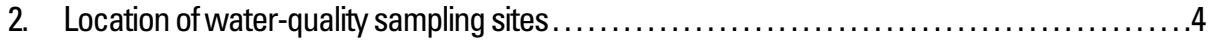

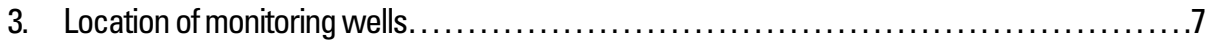

\section{Tables}

1. Values of physical properties and concentrations of inorganic constituents and nutrients in samples

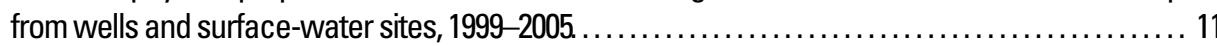

2. Concentrations of trace elements, organic carbon, bromide, and silica in samples from selected monitoring wells, 1999.

3. Organic compounds and pesticides analyzed and minimum reporting limits for samples from

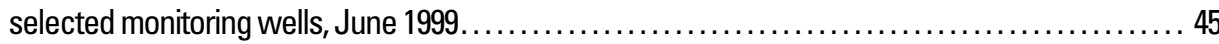

4. Wastewater organic compounds analyzed and minimum reporting limits for samples from selected

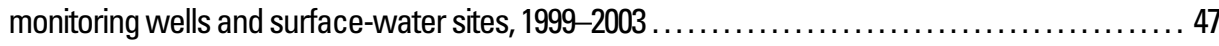

5. Pesticides analyzed and minimum reporting limits for samples from selected monitoring wells and

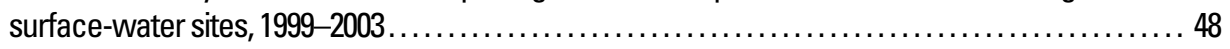

6. Concentrations of wastewater organic compounds and pesticides in samples from selected

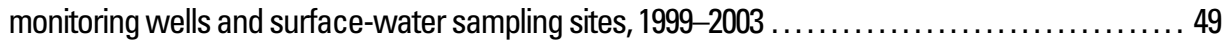

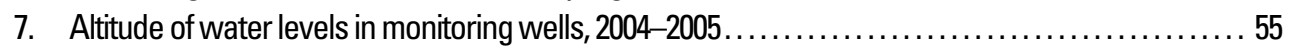




\section{Conversion Factors and Datum}

\begin{tabular}{|c|c|c|}
\hline Multiply & By & To obtain \\
\hline \multicolumn{3}{|c|}{ Length } \\
\hline inch (in.) & 2.54 & centimeter $(\mathrm{cm})$ \\
\hline inch (in.) & 25.4 & millimeter $(\mathrm{mm})$ \\
\hline foot (ft) & 0.3048 & meter $(\mathrm{m})$ \\
\hline mile (mi) & 1.609 & kilometer (km) \\
\hline \multicolumn{3}{|c|}{ Area } \\
\hline acre & 4,047 & square meter $\left(\mathrm{m}^{2}\right)$ \\
\hline acre & 0.4047 & hectare (ha) \\
\hline acre & 0.4047 & square hectometer $\left(\mathrm{hm}^{2}\right)$ \\
\hline acre & 0.004047 & square kilometer $\left(\mathrm{km}^{2}\right)$ \\
\hline square mile $\left(\mathrm{mi}^{2}\right)$ & 259.0 & hectare (ha) \\
\hline square mile $\left(\mathrm{mi}^{2}\right)$ & 2.590 & square kilometer $\left(\mathrm{km}^{2}\right)$ \\
\hline \multicolumn{3}{|c|}{ Flow rate } \\
\hline gallon per minute $(\mathrm{gal} / \mathrm{min})$ & 0.06308 & liter per second $(\mathrm{L} / \mathrm{s})$ \\
\hline gallon per day $(\mathrm{gal} / \mathrm{d})$ & 0.003785 & cubic meter per day $\left(\mathrm{m}^{3} / \mathrm{d}\right)$ \\
\hline million gallons per day (Mgal/d) & 0.04381 & cubic meter per second $\left(\mathrm{m}^{3} / \mathrm{s}\right)$ \\
\hline
\end{tabular}

Horizontal coordinate information is referenced to the North American Datum of 1983 (NAD 83).

Vertical coordinate information is referenced to the National Geodetic Vertical Datum of 1929 (NGVD 29).

Temperature in degrees Celsius $\left({ }^{\circ} \mathrm{C}\right)$ can be converted to degrees Fahrenheit $\left({ }^{\circ} \mathrm{F}\right)$ as follows:

$$
{ }^{\circ} \mathrm{F}=\left(1.8 \mathrm{x}^{\circ} \mathrm{C}\right)+32
$$

Altitude, as used in this report, refers to distance above the vertical datum.

Specific conductance is given in microsiemens per centimeter at 25 degrees Celsius $/ \mu \mathrm{S} / \mathrm{cm}$ at $\left.25^{\circ} \mathrm{C}\right)$.

Concentrations of chemical constituents in water are given either in milligrams per liter (mg/L) or micrograms per liter $(\mu \mathrm{g} / \mathrm{L})$. 


\title{
Water-Quality Data, 1999-2005, and Ground-Water Level Data, 2004-2005, for McBaine Bottoms, Including the Eagle Bluffs Conservation Area, Columbia, Missouri
}

\author{
By Brenda J. Smith and Joseph M. Richards
}

\section{Abstract}

The U.S. Geological Survey, in cooperation with the city of Columbia, Missouri, and the Missouri Department of Conservation, collected ground-water quality data from June 1999 through August 2005, surface-water quality data from August 1999 through August 2003, and water-level data from February 2004 through August 2005 in McBaine Bottoms, southwest of Columbia. McBaine Bottoms, adjacent to the Missouri River, is the location of the municipal-supply well field for the city of Columbia, the city of Columbia wastewater-treatment wetlands, and the Missouri Department of Conservation Eagle Bluffs Conservation Area.

This report presents water-quality data, which include water-quality analyses of samples collected from 36 waterquality sampling sites ( 31 were wells and 5 were surface-water sites), and ground-water level data, which include water-level measurements from more than 80 wells. Water samples were analyzed for physical properties, inorganic chemical constituents, nutrients, and dissolved iron. Selected samples were analyzed for trace elements, wastewater organic compounds, and pesticides.

In samples from monitoring wells, chloride concentrations ranged from 2.41 to $259 \mathrm{mg} / \mathrm{L}$ (milligrams per liter), sodium concentrations ranged from 1.08 to $175 \mathrm{mg} / \mathrm{L}$, and sulfate concentrations ranged from less than 0.2 to $271 \mathrm{mg} / \mathrm{L}$ (all concentrations were dissolved). Dissolved nitrite plus nitrate as nitrogen concentrations ranged from less than 0.05 to $0.46 \mathrm{mg} / \mathrm{L}$. Total phosphorous concentrations ranged from less than 0.04 to $1.68 \mathrm{mg} / \mathrm{L}$, dissolved phosphorous concentrations ranged from less than 0.04 to $1.50 \mathrm{mg} / \mathrm{L}$, and dissolved orthophosphorous concentrations ranged from less than 0.01 to $1.83 \mathrm{mg} / \mathrm{L}$. Dissolved iron concentrations ranged from less than 6 to $42,900 \mu \mathrm{g} / \mathrm{L}$ (micrograms per liter). Dissolved arsenic concentrations in samples from two monitoring wells ranged from 11 to $37 \mu \mathrm{g} / \mathrm{L}$.

In samples from surface-water sampling sites, chloride concentrations ranged from 8.67 to $289 \mathrm{mg} / \mathrm{L}$, sodium concentrations ranged from 6.18 to $219 \mathrm{mg} / \mathrm{L}$, and sulfate concentrations ranged from 33.4 to $119 \mathrm{mg} / \mathrm{L}$. All of the minimum concentrations were detected in samples from Perche Creek. Dissolved nitrite plus nitrate as nitrogen concentrations ranged from less than 0.05 to $1.53 \mathrm{mg} / \mathrm{L}$. Total phosphorous concentra- tions ranged from 0.07 to $3.06 \mathrm{mg} / \mathrm{L}$, dissolved phosphorous concentrations ranged from less than 0.05 to $2.88 \mathrm{mg} / \mathrm{L}$, and dissolved orthophosphorous concentrations ranged from less than 0.01 to $2.86 \mathrm{mg} / \mathrm{L}$. Most of the maximum concentrations were detected in samples from the city outflow.

More than 35 wastewater organic compounds and pesticides were detected in samples from the city outflow. However, most concentrations were estimated or their presence verified, but not quantified.

Water levels in monitoring wells ranged from 548.54 to $576.55 \mathrm{ft}$ (feet) above the National Geodetic Vertical Datum of 1929 (NGVD 29). The difference in water levels from February 2004 to August 2005 ranged from $0.13 \mathrm{ft}$ to $10.97 \mathrm{ft}$.

\section{Introduction}

When expansion of the Columbia, Missouri, wastewatertreatment facility became necessary, the construction of a wastewater-treatment wetland, hereafter referred to as the treatment wetland, was constructed in McBaine Bottoms (fig. 1) as an alternative to expanding the existing activated sludge facilities. The Missouri Department of Conservation uses the treated effluent from the treatment wetland as a water source for the 1,300-acre managed wetland on the 4,200-acre Eagle Bluffs Conservation Area (fig. 1). The city of Columbia municipalsupply well field, also in McBaine Bottoms, is north of the Eagle Bluffs Conservation Area and west of the treatment wetland.

The treatment wetland consists of four units with a total surface area of about 130 acres. Wastewater entering the treatment wetland consists of blended primary and secondary treated effluent from the wastewater-treatment facility. The design capacity of the treatment wetland is $20 \mathrm{Mgal} / \mathrm{d}$ (million gallons per day), with an average treated effluent of about 16 $\mathrm{Mgal} / \mathrm{d}$ (City of Columbia, 2005). When the treatment wetland began treating wasterwater, the city of Columbia stopped using Perche Creek as the receiving water for the treated effluent (Richards, 2002).

Before the operations began at the treatment wetland, monitoring wells were drilled from 1991 through 1993 by the city of Columbia and the U.S. Geological Survey, and samples 


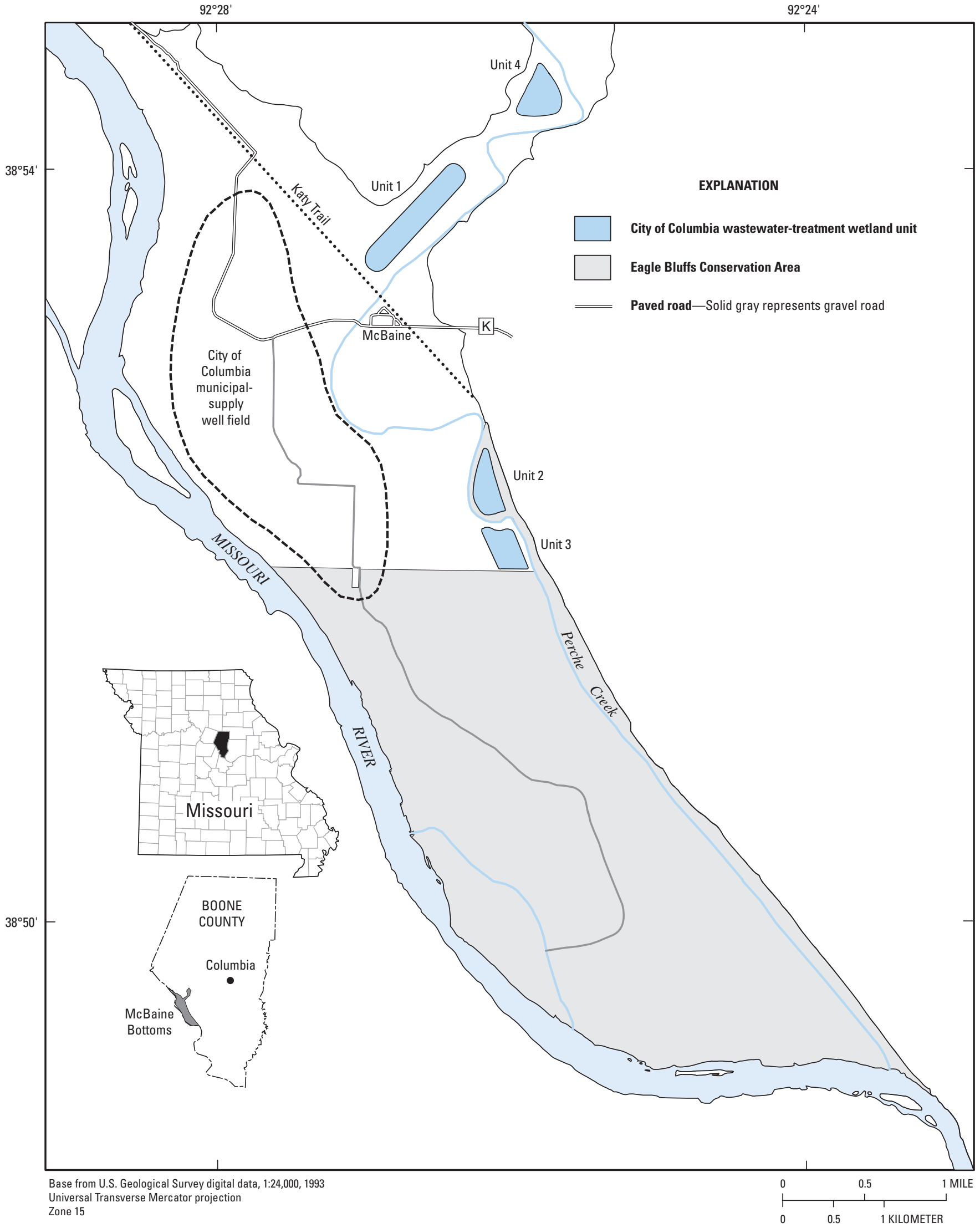

Figure 1. Location of McBaine Bottoms, Columbia, Missouri. 
were collected from the wells for water-quality analyses (Richards, 1995, 1999). The Eagle Bluffs Conservation Area began accepting treated effluent in 1994, and full wetland management began in late 1995. After effluent discharge began in 1994, samples from a shallow monitoring well, (MW1-2A, fig. 2) about $30 \mathrm{ft}$ (feet) deep near treatment wetland unit 1 (fig. 1), began showing gradual but marked concentration increases in various water-quality constituents. Soon after, samples from other monitoring wells on the Eagle Bluffs Conservation Area began showing similar changes. The water-quality constituents showing the most substantial changes were dissolved sodium, potassium, calcium, sulfate, and chloride (Richards, 1999, 2002).

In 2000, the U.S. Geological Survey installed 55 shallow monitoring wells throughout McBaine Bottoms. These wells were installed to determine the ground-water flow and groundand surface-water interaction at McBaine Bottoms (Smith, 2003). Shortly after the wells were installed, one was destroyed.

The city of Columbia [population of 84,531 (U.S. Census Bureau, 2000)] uses the Missouri River alluvial aquifer in McBaine Bottoms for its municipal water supply and currently (2006) pumps water from seven well pairs. Each well pair consists of two, 4-ft diameter wells about $100 \mathrm{ft}$ deep with pumps rated at about 2,000 gallons per minute.

The purpose of this report is to present the water-quality data from samples collected from June 1999 through August 2005 from 36 water-quality sampling sites [data collected before June 1999 are given in Richards (1995, 1999, and 2002)] and to present ground-water level data for wells from 2004 through 2005 [data collected before 2004 are given in Smith (2003)]. Physical properties, inorganic constituent concentrations (including iron), and nutrient concentrations were determined at all sampling sites. Densities of an indicator bacteria (fecal coliform) were determined for samples collected from 1999 through 2002. Concentrations of trace elements, wastewater organic compounds, and pesticides were measured for selected samples collected from 1999 through 2003.

\section{Study Area}

McBaine Bottoms is part of the Missouri River alluvial valley about $7 \mathrm{mi}$ (miles) southwest of Columbia, Missouri. It is an $8.7-\mathrm{mi}^{2}$ (square mile) area bounded to the south and west by the Missouri River and to the north and east by the Missouri River bluffs. Perche Creek flows southward along the Missouri River bluffs and discharges to the Missouri River in the southeast part of the study area (fig. 1). Before the treatment wetland was fully operational, the city of Columbia used Perche Creek as the discharge point for treated sewage effluent (Richards, 2002). McBaine Bottoms, including parts of the Eagle Bluffs Conservation Area, is agricultural land primarily used for the cultivation of row crops, such as corn and soybeans. McBaine Bottoms receives an average of 38 in. (inches) of precipitation annually (Missouri Department of Natural Resources, 1986).
Land-surface altitudes range from $580 \mathrm{ft}$ above the National Geodetic Vertical Datum of 1929 (NGVD 29) in the northwestern part of the study area to $550 \mathrm{ft}$ in the extreme southeastern part of McBaine Bottoms. The altitude of a bluff west of McBaine Bottoms that overlooks the Missouri River and the Missouri River alluvial plain is as much as $800 \mathrm{ft}$ above the NGVD 29 (Smith, 2003).

The alluvium underlying McBaine Bottoms is composed of silt, clay, coarse-grained sand, and gravel. The maximum thickness of the alluvium is about $95 \mathrm{ft}$; the average saturated thickness is about $60 \mathrm{ft}$ (Emmett and Jeffrey, 1969). The sand and gravel in the lower part of the alluvium form the alluvial aquifer (Richards, 1999).

\section{Water-Quality Data}

Water samples were collected from 31 wells (fig. 2) from June 1999 through August 2005 and from 5 surface-water sampling sites from August 1999 through August 2003. The samples were analyzed for constituents at the U.S. Geological Survey (USGS) National Water Quality Laboratory (NWQL). Quality-assurance/quality-control (QA/QC) samples were collected and analyzed to evaluate the bias and variability associated with environmental data and interpretation of analytical results.

\section{Sampling Methods}

Samples were collected and processed as described in Richards (1995, 1999). The water level, specific conductance, $\mathrm{pH}$, temperature, dissolved oxygen, and bacteria densities were determined onsite at the time of sampling, after physical properties had stabilized (usually after pumping about two well volumes); inorganic constituent, nutrient, and organic constituent concentrations were determined by the NWQL (table 1, at the back of this report). Samples were analyzed for dissolved constituents, unless otherwise noted in the tables. Samples were analyzed according to methods described by Wershaw and others (1983), Fishman and Friedman (1989), Patton and Truitt (1992), and Fishman (1993).

The water levels listed in table 1 were measured immediately before the water in the well was pumped. Specific conductance, $\mathrm{pH}$, temperature, and dissolved oxygen were determined onsite using procedures described by Wilde and Radtke (1998). Specific conductance values were measured using a portable conductivity meter with temperature compensation designed to express readings in microsiemens per centimeter at $25^{\circ} \mathrm{C}$ (degrees Celsius). The $\mathrm{pH}$ value was measured at the time of sample collection with an electronic meter calibrated with buffers bracketing the expected $\mathrm{pH}$ values of the samples. The water temperature was determined with the same meter that determined the $\mathrm{pH}$ value. Dissolved oxygen concentrations were determined using a low-range colorimetric method or a portable temperature compensated meter. 


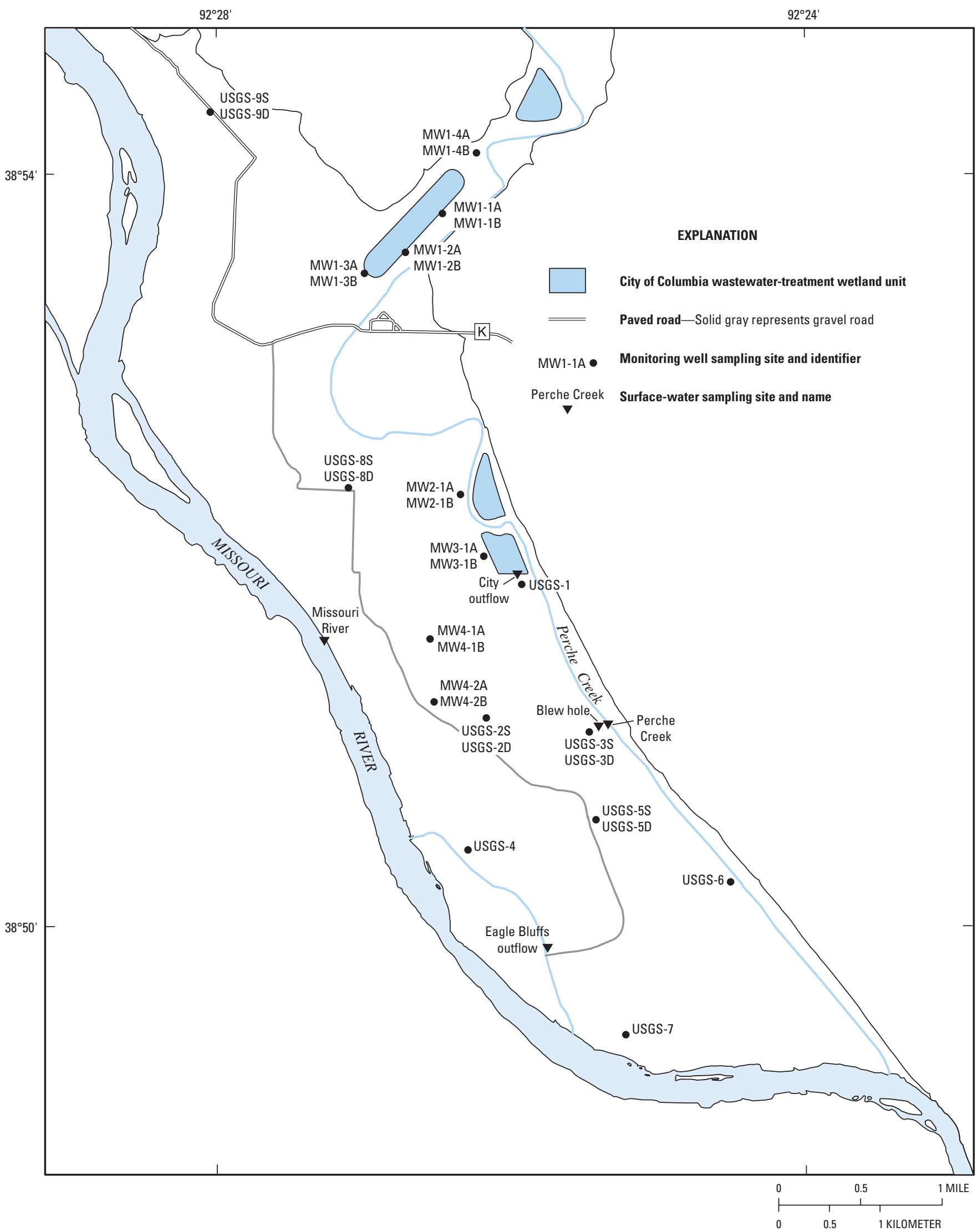

Figure 2. Location of water-quality sampling sites. 
The components of analytical variability (uncertainty) in water-quality results can be estimated when QA/QC samples of the correct types and quantities are incorporated into measurement procedures. The USGS NWQL incorporates numerous QA/QC samples in the laboratory to ensure that the measurement system is functioning properly. In addition to the laboratory QA/QC procedures, replicate and blank samples were collected onsite at McBaine Bottoms. Replicate samples are collected and analyzed to determine the precision of sampling, processing, and onsite analysis. Generally replicate samples were collected immediately after a regular sample using the same equipment and sampling techniques. Blank samples were used to determine that equipment cleaning, sample collection, and processing procedures provided an inconsequential source of bias to the regular samples. For the blank samples, highly purified water was passed through the same equipment used to collect and process the water-quality samples and then stored and shipped using the same methods that were used for the water-quality samples. Regular, replicate, and blank samples were analyzed at the USGS NWQL using identical analytical techniques. A relative percent difference less than 20 percent between the regular sample and the replicate usually indicates that the sampling process is representative. Replicate samples analyses are listed in the table adjacent to the regular sample. Generally the relative percent difference was less than 20 percent between the regular samples and the replicate samples collected at McBaine Bottoms. Most compounds were not detected in any blank samples; if detected, the concentrations were near the reporting limit for the compound (data on file at the U.S. Geological Survey, Rolla, Missouri).

\section{Ground-Water Quality}

Water-quality samples were collected from 31 wells (fig. 2 ). Twenty-six of the monitoring wells that were sampled were in well pairs; the well pairs are identified with the suffix A, B, $\mathrm{S}$, or D. For each well pair, the shallow well (identified by A or $\mathrm{S}$ ) is about $30 \mathrm{ft}$ deep and the deep well (identified by B or D) is about $60 \mathrm{ft}$ deep. Wells USGS-1 and USGS- 6 are about $30 \mathrm{ft}$ deep, USGS-4 and USGS-7 are about $60 \mathrm{ft}$ deep, and the city of Columbia public-supply well 5 is $104 \mathrm{ft}$ deep. Samples were collected at monitoring wells designated by the prefix MW1through MW4- from June 1999 through August 2005; samples were collected at monitoring wells designated by the prefix USGS- from August 1999 through April 2003; and samples were collected at public-supply well 5 during 2003.

Constituent concentrations that have shown the most substantial changes since the operation of the wetland were dissolved sodium, potassium, calcium, sulfate, and chloride (Richards, 1999, 2002). The following concentrations were determined in samples collected from June 1999 through August 2005: calcium concentrations ranged from 77.1 to 226 $\mathrm{mg} / \mathrm{L}$ (milligrams per liter), chloride concentrations ranged from 2.41 to $259 \mathrm{mg} / \mathrm{L}$, potassium concentrations ranged from 1.59 to $8.60 \mathrm{mg} / \mathrm{L}$, sodium concentrations ranged from 1.08 to
$175 \mathrm{mg} / \mathrm{L}$, and sulfate concentrations ranged from less than 0.2 to $271 \mathrm{mg} / \mathrm{L}$ (all concentrations were dissolved). The Missouri secondary maximum contaminant level for drinking water for chloride and sulfate is $250 \mathrm{mg} / \mathrm{L}$ (Missouri Department of Natural Resources, 2005).

Nutrients (nitrogen and phosphorous species) are of particular interest in a wetland environment, especially one in which the source of water is treated sewage effluent. The concentration of dissolved ammonia as nitrogen ranged from less than 0.02 to $4.05 \mathrm{mg} / \mathrm{L}$ (milligrams per liter). Dissolved nitrite plus nitrate as nitrogen concentrations ranged from less than 0.05 to $0.46 \mathrm{mg} / \mathrm{L}$. Total phosphorous concentrations ranged from less than 0.04 to $1.68 \mathrm{mg} / \mathrm{L}$, dissolved phosphorous concentrations ranged from less than 0.04 to $1.50 \mathrm{mg} / \mathrm{L}$, and dissolved orthophosphorous concentrations ranged from less than 0.01 to 1.83 $\mathrm{mg} / \mathrm{L}$.

Fecal coliform densities were determined for sampling sites from 1999 through 2002 (data on file at the U.S. Geological Survey, Rolla, Missouri). Densities in samples from monitoring wells were less than $5 \mathrm{col} / 100 \mathrm{~mL}$ (colonies per $100 \mathrm{mil}$ liliters). However, during sampling on August 23 through 25, 2000 , densities in samples from seven monitoring wells ranged from an estimated 7 to an estimated $150 \mathrm{col} / 100 \mathrm{~mL}$. These wells included monitoring wells MW3-1A, MW4-1A, MW42A, USGS-4, USGS-5S, USGS-6, and USGS-7 (fig. 2).

Dissolved trace-element concentrations were determined in selected samples from monitoring wells collected in June and September 1999 (table 2, at the back of this report), except for iron. Iron concentrations were determined for all samples collected from 1999 to 2005 (table 1) and ranged from less than 6 to $42,900 \mu \mathrm{g} / \mathrm{L}$ (micrograms per liter). Arsenic concentrations were less than $10 \mu \mathrm{g} / \mathrm{L}$ in all samples except for those from monitoring wells MW1-3A and MW1-4B. In samples from these wells, the concentrations ranged from 11 to $37 \mu \mathrm{g} / \mathrm{L}$. Concentrations of beryllium, cadmium, chromium, copper, lead, molybdenum, and silver were less than the reporting limit. Detected concentrations for mercury, nickel, selenium, vanadium, and zinc were at the reporting limit or were estimated less than the reporting limit (table 2).

In June 1999, water-quality samples were collected at five monitoring wells (MW1-3B, MW1-4A, MW2-1A, MW4-1A, and USGS-9S) for the determination of organic compounds and pesticides. The samples were analyzed for concentrations of selected organic compounds and pesticides (table 3, at the back of this report). No constituent concentrations were detected in these samples. Concentrations of wastewater organic compounds and pesticides were determined in samples from selected monitoring wells from 1999 through 2003. A list of wastewater organic compounds (table 4, at the back of this report) and pesticides (table 5, at the back of this report) that were analyzed and their minimum reporting limits are given. The concentrations of wastewater organic compounds and pesticides are listed in table 6, at the back of the report. Six wastewater organic compounds and pesticides were detected in samples from monitoring wells MW3-1A and USGS-3S, and seven in samples from monitoring well MW1-3A. Detected concen- 
trations were estimated or their presence verified, but not quantified. The exceptions were atrazine concentrations of 0.007 $\mu \mathrm{g} / \mathrm{L}$ detected in a water sample from monitoring well MW4$1 \mathrm{~A}$ and $0.008 \mu \mathrm{g} / \mathrm{L}$ detected in a sample from monitoring well MW1-3B and 10 phenol concentrations that ranged from 0.5 to $1.8 \mu \mathrm{g} / \mathrm{L}$. The phenol concentrations were detected in samples from monitoring wells MW1-1A, MW1-3A, MW1-4A, MW31A, MW4-1A, MW4-2A, USGS-3S, and USGS-9D.

\section{Surface-Water Quality}

The five surface-water sites (fig. 2) include a scour hole caused by levee failures during past flooding that has formed along the levee surrounding the study area (blew hole), the outflow from the treatment wetlands (city outflow), the outflow from the Eagle Bluffs Conservation Area (Eagle Bluffs outflow), a site on Perche Creek near the blew hole (Perche Creek), and a site on the Missouri River near the Eagle Bluffs Conservation Area (Missouri River). Samples were collected at these sites from August 1999 through August 2003, except for the Missouri River site where samples were collected during 2003.

Calcium concentrations ranged from 31.6 to $104 \mathrm{mg} / \mathrm{L}$, chloride concentrations ranged from 8.67 to $289 \mathrm{mg} / \mathrm{L}$, potassium concentrations ranged from 3.91 to $41.5 \mathrm{mg} / \mathrm{L}$, sodium concentrations ranged from 6.18 to $219 \mathrm{mg} / \mathrm{L}$, and sulfate concentrations ranged from 33.4 to $119 \mathrm{mg} / \mathrm{L}$ (table 1 ; all concentrations were dissolved). All of the minimum concentrations were detected in samples from Perche Creek.

Nutrient concentrations were measured in samples collected from the surface-water sites (table 1). The concentration of dissolved ammonia as nitrogen ranged from less than 0.02 to an estimated concentration of $14.6 \mathrm{mg} / \mathrm{L}$. Dissolved nitrite plus nitrate as nitrogen concentrations ranged from less than 0.05 to $1.53 \mathrm{mg} / \mathrm{L}$. Total phosphorous concentrations ranged from 0.07 to $3.06 \mathrm{mg} / \mathrm{L}$, dissolved phosphorous concentrations ranged from less than 0.05 to $2.88 \mathrm{mg} / \mathrm{L}$, and dissolved orthophosphorous concentrations ranged from less than 0.01 to $2.86 \mathrm{mg} / \mathrm{L}$. Most of the maximum concentration were detected in samples from the city outflow.

Densities of fecal coliform bacteria in samples from the surface-water sites were larger than those in samples from monitoring wells. The densities ranged from less than $1 \mathrm{col} / 100 \mathrm{~mL}$ to an estimated $57,000 \mathrm{col} / 100 \mathrm{~mL}$ at the city outflow (data on file at the U.S. Geological Survey, Rolla, Missouri).

More than 35 wastewater organic compounds and pesticides were detected in samples from the city outflow (table 5). However, most concentrations were estimated or their presence verified, but not quantified. Atrazine concentrations in samples from three of the surface-water sampling sites (blew hole, city outflow, and Perche Creek) ranged from 0.008 to $0.192 \mu \mathrm{g} / \mathrm{L}$ Diazinon concentrations in samples from three of the surface-water sampling sites (city outflow, Eagle Bluffs outflow, and Perche Creek) ranged from 0.012 to an estimated $0.100 \mu \mathrm{g} / \mathrm{L}$.

\section{Ground-Water Level Data}

Ground-water levels were measured in more than 80 wells in McBaine Bottoms (fig. 3). They included the 30 wells that were sampled for water-quality analyses, the wells that were installed in 2000, and additional wells that included 2 wells installed by the city of Columbia and 2 wells installed by the U.S. Geological Survey. Initially, 55 wells were installed in 2000, but shortly thereafter, monitoring well MW-149 was destroyed. The altitude of monitoring well measuring points (usually the top of the riser pipe) was surveyed so that water levels could be accurately referenced to a common datum (NGVD 29). An electric tape was lowered into the well, and the depth to the ground water from the measuring point was read to the nearest $0.01 \mathrm{ft}$. Water-level measurements were made in February and August 2004 and March and August 2005 (table 7, at the back of this report). Water-level measurements at all sites were made during the same day so the Missouri River stage fluctuations or temporal ground-water fluctuations would have a minimal effect on the measurements.

Water levels ranged from $548.54 \mathrm{ft}$ above NGVD 29 in monitoring well MW-147 to $576.55 \mathrm{ft}$ above NGVD 29 in monitoring well MW-142. The difference in water levels from February 2004 to August 2005 ranged from $0.13 \mathrm{ft}$ at MW-150 to $10.97 \mathrm{ft}$ at MW-152.

\section{Summary}

The U.S. Geological Survey, in cooperation with the city of Columbia, Missouri, and the Missouri Department of Conservation, collected ground-water quality data from June 1999 through August 2005, surface-water quality data from August 1999 through August 2003, and water-level data from February and August 2004 and March and August 2005 in McBaine Bottoms, southwest of Columbia. McBaine Bottoms, adjacent to the Missouri River, is the location of the municipal-supply well field for the city of Columbia, the city of Columbia wastewatertreatment wetlands, and the Missouri Department of Conservation Eagle Bluffs Conservation Area.

This report presents water-quality data, which include water-quality analyses of samples collected from 36 waterquality sampling sites ( 31 were wells and 5 were surface-water sites), and ground-water level data, which include water-level measurements from more than 80 wells. Water samples were analyzed for physical properties, inorganic chemical constituents, nutrients, and dissolved iron. Selected samples were analyzed for trace elements, wastewater organic compounds, and pesticides.

In samples from monitoring wells, calcium concentrations ranged from 77.1 to $226 \mathrm{mg} / \mathrm{L}$ (milligrams per liter), chloride concentrations ranged from 2.41 to $259 \mathrm{mg} / \mathrm{L}$, potassium concentrations ranged from 1.59 to $8.60 \mathrm{mg} / \mathrm{L}$, sodium concentrations ranged from 1.08 to $175 \mathrm{mg} / \mathrm{L}$, and sulfate concentrations ranged from less than 0.2 to $271 \mathrm{mg} / \mathrm{L}$ (all concentrations were 


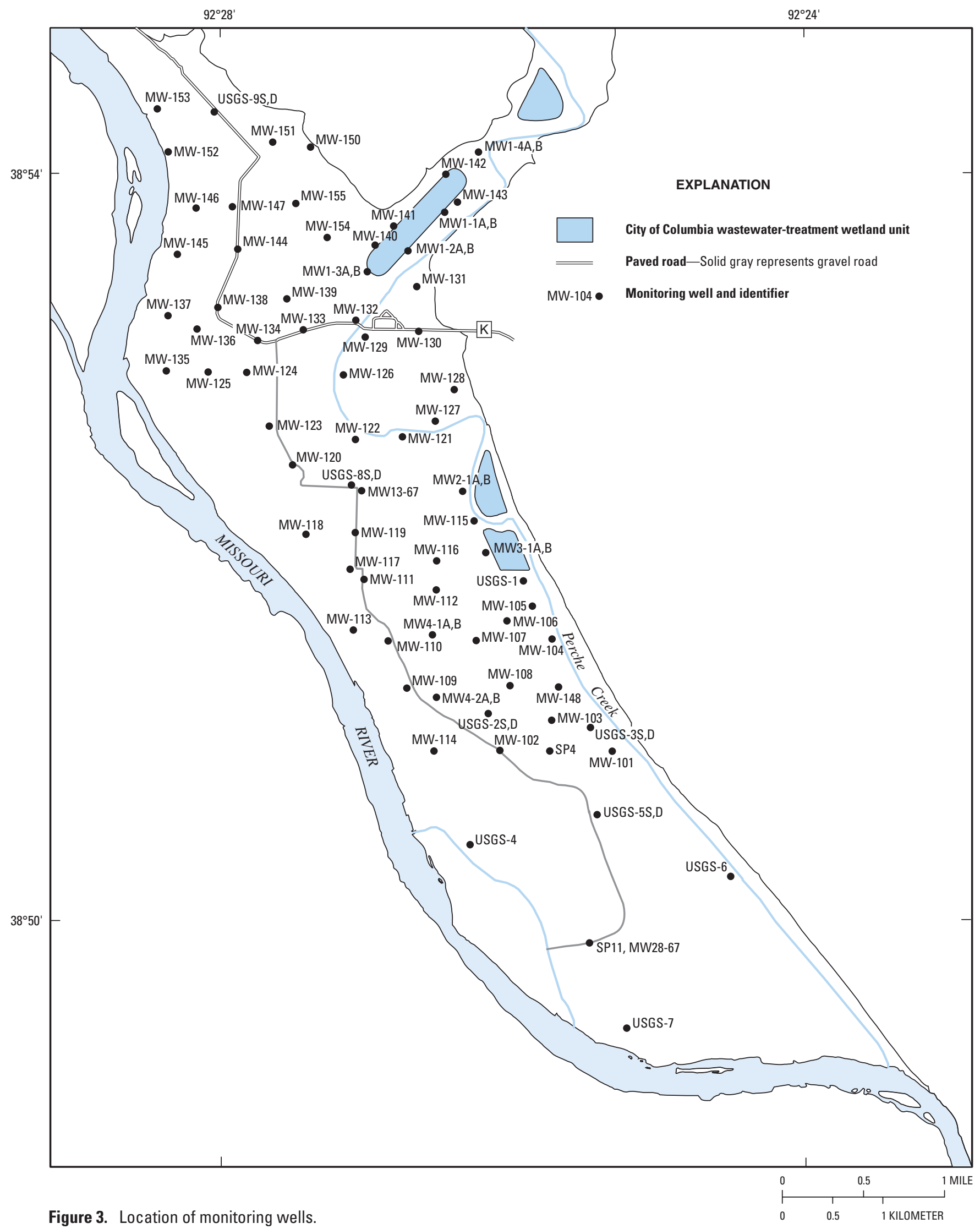




\section{Water-Quality Data, 1999-2005, and Ground-Water Level Data, 2004-2005, for McBaine Bottoms, Columbia, Missouri}

dissolved). Dissolved nitrite plus nitrate as nitrogen concentrations ranged from less than 0.05 to $0.46 \mathrm{mg} / \mathrm{L}$. Total phosphorous concentrations ranged from less than 0.04 to $1.68 \mathrm{mg} / \mathrm{L}$, dissolved phosphorous concentrations ranged from less than 0.04 to $1.50 \mathrm{mg} / \mathrm{L}$, and dissolved orthophosphorous concentrations ranged from less than 0.01 to $1.83 \mathrm{mg} / \mathrm{L}$. Dissolved iron concentrations ranged from less than 6 to $42,900 \mu \mathrm{g} / \mathrm{L}$ (micrograms per liter). Dissolved arsenic concentrations in samples from two monitoring wells ranged from 11 to $37 \mu \mathrm{g} / \mathrm{L}$.

In samples from surface-water sampling sites, calcium concentrations ranged from 31.6 to $104 \mathrm{mg} / \mathrm{L}$, chloride concentrations ranged from 8.67 to $289 \mathrm{mg} / \mathrm{L}$, potassium concentrations ranged from 3.91 to $41.5 \mathrm{mg} / \mathrm{L}$, sodium concentrations ranged from 6.18 to $219 \mathrm{mg} / \mathrm{L}$, and sulfate concentrations ranged from 33.4 to $119 \mathrm{mg} / \mathrm{L}$ (all concentrations were dissolved). All of the minimum concentrations were detected in samples from Perche Creek. Dissolved nitrite plus nitrate as nitrogen concentrations ranged from less than 0.05 to 1.53 $\mathrm{mg} / \mathrm{L}$. Total phosphorous concentrations ranged from 0.07 to $3.06 \mathrm{mg} / \mathrm{L}$, dissolved phosphorous concentrations ranged from less than 0.05 to $2.88 \mathrm{mg} / \mathrm{L}$, and dissolved orthophosphorous concentrations ranged from less than 0.01 to $2.86 \mathrm{mg} / \mathrm{L}$. Most of the maximum concentration were detected in samples from the city outflow.

More than 35 wastewater organic compounds and pesticides were detected in samples from the city outflow. However, most concentrations were estimated or their presence verified, but not quantified.

Water levels in monitoring wells ranged from $548.54 \mathrm{ft}$ to $576.55 \mathrm{ft}$ above the National Geodetic Vertical Datum of 1929 (NGVD 29). The difference in water levels from February 2004 to August 2005 ranged from $0.13 \mathrm{ft}$ to $10.97 \mathrm{ft}$.

\section{References}

City of Columbia, 2005, Columbia Regional Wastewater Treatment Plant, accessed on July 7, 2005, at http:// wwwgocolumbiamo.com/PublicWorks/Sewer/ wwtppg_4.html.

Emmett, L.F., and Jeffrey, H.G., 1969, Reconnaissance of the ground-water resources of the Missouri River alluvium between Jefferson City and Miami, Missouri: U.S. Geological Survey Hydrologic Investigations Atlas HA-340, 1 sheet.

Fishman, M.J., ed., 1993, Methods of analysis by the U.S. Geological Survey National Water Quality Laboratory-Determination of inorganic and organic constituents in water and fluvial sediments: U.S. Geological Survey Open-File Report 93-125, $217 \mathrm{p}$.

Fishman, M.J., and Friedman, L.C., 1989, Methods for determination of inorganic substances in water and fluvial sediments: U.S. Geological Survey Techniques of WaterResources Investigations, book 5, chap. A1, 545 p.

Missouri Department of Natural Resources, 1986, Missouri water atlas: Jefferson City, 100 p.
Missouri Department of Natural Resources, 2005, Code of state regulations-Chapter 4, Contaminant levels and monitoring: accessed September 2005 at URL http:// www.sos.state.mo.us/adrules/csr/current/10csr/10c60-4.pdf.

Patton, C.J., and Truitt, W.P., 1992, Methods of analysis by the U.S. Geological Survey National Water Quality Laboratory-Determination of total phosphorus by a Kjeldahl digestion method and an automated colorimetric finish that includes dialysis: U.S. Geological Survey Open-File Report 92-146, 39 p.

Richards, J.M., 1995, Hydrologic data for the Columbia/Eagle Bluffs Wetland Complex, Columbia, Missouri-1992-93: U.S. Geological Survey Open-File Report 95-109, 50 p.

Richards, J.M., 1999, Hydrologic data for the Columbia/Eagle Bluffs Wetland Complex, Columbia, Missouri-1993-99: U.S. Geological Survey Open-File Report 99-607, 91 p.

Richards, J.M., 2002, Water-quality and ground-water hydrology of the Columbia/Eagle Bluffs Wetland Complex, Columbia, Missouri-1992-99: U.S. Geological Survey Water-Resources Investigations Report 02-4227, 131 p.

Smith, B.J., 2003, Ground-water flow and ground- and surfacewater interaction at McBaine Bottoms, Columbia, Missouri-2000-02: U.S. Geological Survey Water-Resources Investigations Report 03-4234, 83 p.

U.S. Census Bureau, 2000, Population estimates program, population division: accessed March 2002 at URL http:// www.gocolumbiamo.com/About_Columbia/demographics.html.

Wershaw, R.L., Fishman, J., Grabbe, R.R., and Lowe, Le.E., eds., 1983, Methods for the determination of organic substances in water and fluvial sediments: U.S. Geological Survey Techniques of Water-Resources Investigations, book 5, chap. A3, 80 p.

Wilde, F.D., and Radtke, D.B., eds., 1998, National field manual for the collection of water-quality data: Field measurements: U.S. Geological Survey Techniques of WaterResources Investigations, book 9, chap. A6, variously paginated. 
Tables

$\longrightarrow$ 

Table 1. Values of physical properties and concentrations of inorganic constituents and nutrients in samples from wells and surface-water sites, $1999-2005$.

$\left[\mu \mathrm{S} / \mathrm{cm}\right.$, microsiemens per centimeter at 25 degrees Celsius; $\mathrm{pH}$, in standard units; ${ }^{\circ} \mathrm{C}$, degrees Celsius; constituent concentrations are dissolved, unless otherwise noted; mg/L, milligrams per liter; $\mathrm{CaCO}_{3}$, calcium carbonate; ANC, total acid neutralizing capacity, incremental titration; N, nitrogen; $\mu \mathrm{g} / \mathrm{L}$, micrograms per liter; NGVD 29, National Geodetic Vertical Datum of 1929; --, no data; $<$, less than; E, estimated; *, replicate sample; >, greater than]

\begin{tabular}{|c|c|c|c|c|c|c|c|c|c|c|c|c|c|}
\hline Date & Time & $\begin{array}{c}\text { Waterlevel } \\
\text { (feet below } \\
\text { measuring } \\
\text { point) }\end{array}$ & $\begin{array}{c}\text { Specific } \\
\text { conductance } \\
(\mu \mathrm{S} / \mathrm{cm})\end{array}$ & pH & $\begin{array}{c}\text { Temperature } \\
\left({ }^{\circ} \mathbf{C}\right)\end{array}$ & $\begin{array}{c}\text { Dissolved } \\
\text { oxygen } \\
\text { (mg/L) }\end{array}$ & $\begin{array}{c}\text { Hardness } \\
\text { (mg/L as } \\
\mathrm{CaCO}_{3} \text { ) }\end{array}$ & $\begin{array}{c}\text { Calcium } \\
\text { (mg/L) }\end{array}$ & $\begin{array}{l}\text { Magnesium } \\
(\mathrm{mg} / \mathrm{L})\end{array}$ & $\begin{array}{c}\text { Sodium } \\
\text { (mg/L) }\end{array}$ & $\begin{array}{l}\text { Potassium } \\
\text { (mg/L) }\end{array}$ & $\begin{array}{l}\text { Alkalinity, } \\
\text { fixed } \\
\text { endpoint } \\
\text { (mg/L) }\end{array}$ & $\begin{array}{l}\text { ANC } \\
\text { (mg/L) }\end{array}$ \\
\hline \multicolumn{14}{|c|}{ MW1-1A (measuring point altitude 578.50 feet above NGVD 29) } \\
\hline 06-03-99 & 1040 & 7.51 & 883 & 6.7 & 15.2 & $<0.1$ & 380 & 119 & 21.0 & 24.2 & 2.72 & -- & 303 \\
\hline 09-02-99 & 1000 & -- & 928 & 6.6 & 15.2 & $<.1$ & 440 & 138 & 23.2 & 24.5 & 3.00 & -- & 314 \\
\hline 03-07-00 & 1240 & 10.29 & 923 & 6.6 & 16.4 & $<.1$ & 400 & 124 & 22.0 & 22.5 & 2.90 & 303 & 306 \\
\hline $08-24-00$ & 1450 & 10.07 & 899 & 6.5 & 16.6 & $<.1$ & 380 & 119 & 21.1 & 24.7 & 2.80 & 281 & 287 \\
\hline $02-21-01$ & 1020 & 10.07 & 852 & 6.5 & 15.4 & $<.1$ & 400 & 124 & 21.6 & 25.0 & 2.56 & 277 & 276 \\
\hline 08-28-01 & 1320 & 9.45 & 970 & 6.6 & 15.9 & $<.1$ & 420 & 132 & 23.0 & 25.2 & 2.96 & 296 & 292 \\
\hline $02-28-02$ & 1010 & 9.75 & 893 & 6.7 & 14.3 & $<.1$ & 380 & 120 & 20.4 & 25.1 & 2.72 & 269 & 269 \\
\hline 08-20-02 & 1420 & 9.92 & 1,020 & 6.8 & 16.4 & $<.1$ & 430 & 133 & 22.6 & 27.3 & 2.97 & 287 & 275 \\
\hline 04-03-03 & 1100 & 9.67 & 1,060 & 6.6 & 15.6 & $<.1$ & -- & -- & -- & -- & -- & -- & -- \\
\hline $08-28-03$ & 1020 & 11.34 & 1,020 & 6.7 & 15.9 & $<.1$ & -- & -- & -- & -- & -- & -- & -- \\
\hline 02-26-04 & 1320 & 9.44 & 1,040 & 6.7 & 14.9 & $<.1$ & -- & 155 & 26.9 & 27.9 & 3.28 & 316 & 308 \\
\hline $08-25-04$ & 1140 & 9.52 & 1,110 & 6.8 & 15.9 & -- & 530 & 167 & 28.2 & 28.6 & 3.14 & 315 & 309 \\
\hline $03-02-05$ & 1210 & 8.29 & 1,170 & 6.6 & 15.0 & -- & 620 & 192 & 32.8 & 28.4 & 3.41 & 341 & 341 \\
\hline 08-03-05 & 1200 & 10.12 & 1,100 & 6.6 & 15.7 & -- & 472 & 149 & 24.2 & 26.6 & 2.85 & 350 & 352 \\
\hline \multicolumn{14}{|c|}{ MW1-1B (measuring point altitude 578.34 feet above NGVD 29) } \\
\hline 06-03-99 & 1000 & 10.44 & 1,340 & 6.8 & 15.1 & $<.1$ & 660 & 201 & 38.0 & 21.9 & 6.74 & -- & 439 \\
\hline 09-02-99 & 1050 & -- & 1,290 & 6.8 & 15.5 & $<.1$ & 690 & 214 & 38.0 & 21.6 & 7.04 & -- & 444 \\
\hline 03-07-00 & 1330 & 17.32 & 1,270 & 6.9 & 16.1 & $<.1$ & 590 & 179 & 34.3 & 20.2 & 5.88 & 436 & 441 \\
\hline $08-24-00$ & 1540 & 16.71 & 1,270 & 6.7 & 16.9 & $<.1$ & 610 & 186 & 34.8 & 19.7 & 7.14 & 439 & 450 \\
\hline $02-21-01$ & 1100 & 15.70 & 1,180 & 6.7 & 14.0 & $<.1$ & 630 & 193 & 36.1 & 20.4 & 6.39 & 436 & 435 \\
\hline 08-28-01 & 1550 & 15.49 & 1,310 & 6.9 & 16.1 & $<.1$ & 640 & 195 & 36.3 & 20.1 & 6.83 & 439 & 437 \\
\hline $02-28-02$ & 1100 & 16.67 & 1,260 & 7.0 & 14.3 & $<.1$ & 590 & 183 & 33.4 & 19.2 & 6.43 & 438 & 438 \\
\hline 08-20-02 & 1330 & 16.07 & 1,350 & 7.0 & 16.0 & $<.1$ & 650 & 200 & 35.9 & 20.9 & 6.47 & 439 & 407 \\
\hline 02-26-04 & 1400 & 16.20 & 1,230 & 6.9 & 14.8 & -- & 650 & 199 & 36.6 & 20.8 & 6.86 & 427 & 403 \\
\hline $08-25-04$ & 1230 & 16.15 & 1,300 & 7.0 & 16.8 & -- & 680 & 209 & 37.2 & 22.0 & 6.68 & 438 & 421 \\
\hline $03-02-05$ & 1300 & 14.43 & 1,290 & 6.9 & 14.6 & -- & 730 & 226 & 39.5 & 21.2 & 7.00 & 436 & 479 \\
\hline 08-03-05 & 1230 & 16.98 & 1,170 & 6.8 & 15.1 & -- & 647 & 203 & 34.4 & 19.8 & 6.84 & 472 & 476 \\
\hline
\end{tabular}


Table 1. Values of physical properties and concentrations of inorganic constituents and nutrients in samples from wells and surface-water sites, 1999-2005.-Continued

$\left[\mu \mathrm{S} / \mathrm{cm}\right.$, microsiemens per centimeter at 25 degrees Celsius; $\mathrm{pH}$, in standard units; ${ }^{\circ} \mathrm{C}$, degrees Celsius; constituent concentrations are dissolved, unless otherwise noted; mg/L, milligrams per liter; $\mathrm{CaCO}_{3}$, calcium carbonate; ANC, total acid neutralizing capacity, incremental titration; N, nitrogen; $\mu \mathrm{g} / \mathrm{L}$, micrograms per liter; NGVD 29, National Geodetic Vertical Datum of 1929; --, no data;

$<$, less than; E, estimated; *, replicate sample; >, greater than]

\begin{tabular}{|c|c|c|c|c|c|c|c|c|c|c|c|c|c|}
\hline Date & Time & $\begin{array}{l}\text { Sulfate } \\
\text { (mg/L) }\end{array}$ & $\begin{array}{c}\text { Chloride } \\
\text { (mg/L) }\end{array}$ & $\begin{array}{c}\text { Fluoride } \\
\text { (mg/L) }\end{array}$ & $\begin{array}{c}\text { Residue, sum } \\
\text { of constituents } \\
(\mathrm{mg} / \mathrm{L}))\end{array}$ & $\begin{array}{c}\text { Nitrite as N } \\
(\mathrm{mg} / \mathrm{L})\end{array}$ & $\begin{array}{c}\text { Nitrite plus } \\
\text { nitrate as N } \\
\text { (mg/L) }\end{array}$ & $\begin{array}{c}\text { Ammonia as } \\
\mathbf{N} \\
(\mathrm{mg} / \mathrm{L})\end{array}$ & $\begin{array}{c}\text { Ammonia } \\
\text { plus organic } \\
\text { N, total } \\
\text { (mg/L) }\end{array}$ & $\begin{array}{c}\text { Phosphorous, } \\
\text { total } \\
\text { (mg/L) }\end{array}$ & $\begin{array}{c}\text { Phosphorous, } \\
\text { dissolved } \\
\text { (mg/L) }\end{array}$ & $\begin{array}{c}\text { Ortho- } \\
\text { phosphorous } \\
\text { (mg/L) }\end{array}$ & $\begin{array}{c}\text { Iron } \\
(\mu \mathrm{g} / \mathrm{L})\end{array}$ \\
\hline \multicolumn{14}{|c|}{ MW1-1A } \\
\hline 06-03-99 & 1040 & 133 & 11.1 & 0.2 & 566 & 0.036 & $<0.05$ & 1.29 & 1.3 & 1.47 & 1.50 & 1.16 & 25,500 \\
\hline 09-02-99 & 1000 & 158 & 11.0 & .2 & 622 & .025 & $<.05$ & 1.29 & 1.2 & 1.28 & 1.15 & .84 & 26,900 \\
\hline $03-07-00$ & 1240 & 168 & 12.2 & .1 & 560 & .022 & $<.05$ & 1.15 & 1.4 & 1.68 & $<.05$ & .83 & 22,200 \\
\hline $08-24-00$ & 1450 & 159 & 13.4 & .2 & 535 & .024 & $<.05$ & 1.09 & 1.2 & 1.49 & 1.41 & .94 & 22,300 \\
\hline $02-21-01$ & 1020 & 179 & 12.4 & .2 & 556 & E.004 & $<.05$ & 1.12 & 1.2 & 1.40 & .62 & .21 & 22,900 \\
\hline $08-28-01$ & 1320 & 140 & 40.8 & .2 & 564 & E.018 & $<.05$ & E1.23 & 1.3 & 1.46 & 1.45 & E.39 & 23,600 \\
\hline $02-28-02$ & 1010 & 172 & 19.3 & .2 & 547 & .027 & $<.05$ & 1.23 & 1.3 & 1.47 & 1.23 & .73 & 21,900 \\
\hline $08-20-02$ & 1420 & 157 & 34.8 & .2 & 576 & .028 & $<.05$ & 1.23 & 1.4 & 1.45 & 1.35 & .40 & 22,700 \\
\hline 04-03-03 & 1100 & -- & 13.6 & -- & -- & -- & -- & -- & -- & -- & -- & -- & -- \\
\hline $08-28-03$ & 1020 & -- & 22.2 & -- & -- & -- & -- & -- & -- & -- & -- & -- & -- \\
\hline $02-26-04$ & 1320 & 200 & 25.4 & .2 & 641 & .109 & $<.60$ & 1.20 & 1.5 & 1.51 & 1.41 & .81 & 7,830 \\
\hline $08-25-04$ & 1140 & 214 & 27.2 & .2 & 687 & $<.008$ & $<.06$ & 1.26 & 1.5 & 1.44 & .29 & $<.02$ & 28,400 \\
\hline $03-02-05$ & 1210 & 224 & 26.6 & .2 & 744 & $<.008$ & $<.06$ & 1.30 & 1.4 & 1.55 & 1.34 & $<.02$ & 32,500 \\
\hline 08-03-05 & 1200 & 184 & 49.1 & .2 & 675 & $<.008$ & $<.06$ & 1.20 & 1.4 & 1.44 & .70 & $<.02$ & 25,160 \\
\hline \multicolumn{14}{|c|}{ MW1-1B } \\
\hline 06-03-99 & 1000 & 268 & 12.4 & .2 & 904 & .077 & $<.05$ & 2.99 & 2.5 & 1.30 & 1.27 & 1.24 & 36,000 \\
\hline 09-02-99 & 1050 & 271 & 14.4 & .2 & 928 & .065 & $<.05$ & 2.50 & 2.3 & 1.20 & 1.10 & 1.40 & 37,800 \\
\hline $03-07-00$ & 1330 & 257 & 12.3 & .2 & 809 & .018 & $<.05$ & 2.28 & 2.6 & 1.41 & $<.05$ & .90 & 32,600 \\
\hline $08-24-00$ & 1540 & 241 & 12.8 & .2 & 801 & $<.010$ & $<.05$ & 2.20 & 2.4 & 1.34 & 1.09 & $<.01$ & 33,100 \\
\hline $02-21-01$ & 1100 & 251 & 11.5 & .3 & 817 & $<.006$ & $<.05$ & 2.18 & 2.4 & 1.30 & .10 & .02 & 34,300 \\
\hline $08-28-01$ & 1550 & 244 & 11.4 & .2 & 811 & E.009 & $<.05$ & E2.36 & 2.4 & 1.37 & 1.29 & E.10 & 34,100 \\
\hline $02-28-02$ & 1100 & 256 & 12.9 & .2 & 811 & .090 & $<.05$ & 2.22 & 2.4 & 1.35 & 1.23 & .56 & 32,500 \\
\hline $08-20-02$ & 1330 & 245 & 11.5 & .2 & 825 & .085 & $<.05$ & 2.40 & 2.5 & 1.38 & 1.49 & 1.34 & 33,900 \\
\hline 02-26-04 & 1400 & 241 & 12.6 & .2 & 816 & .095 & $<.60$ & 2.14 & 2.5 & 1.41 & .99 & 1.13 & 36,500 \\
\hline $08-25-04$ & 1230 & 242 & 12.1 & .2 & 833 & $<.008$ & $<.06$ & 4.05 & 2.4 & 1.38 & .92 & $<.02$ & 35,700 \\
\hline $03-02-05$ & 1300 & 247 & 12.5 & .2 & 879 & $<.008$ & $<.06$ & 3.64 & 2.4 & 1.36 & 1.11 & $<.02$ & 33,260 \\
\hline 08-03-05 & 1230 & 247 & 12.7 & .2 & 842 & E.004 & $<.06$ & 2.18 & 2.4 & 1.34 & .26 & $<.02$ & 30,760 \\
\hline
\end{tabular}


Table 1. Values of physical properties and concentrations of inorganic constituents and nutrients in samples from wells and surface-water sites, 1999-2005.-Continued

$\left[\mu \mathrm{S} / \mathrm{cm}\right.$, microsiemens per centimeter at 25 degrees Celsius; $\mathrm{pH}$, in standard units; ${ }^{\circ} \mathrm{C}$, degrees Celsius; constituent concentrations are dissolved, unless otherwise noted; mg/L, milligrams per liter; $\mathrm{CaCO}_{3}$, calcium carbonate; ANC, total acid neutralizing capacity, incremental titration; N, nitrogen; $\mu \mathrm{g} / \mathrm{L}$, micrograms per liter; NGVD 29, National Geodetic Vertical Datum of 1929; --, no data; $<$, less than; E, estimated; *, replicate sample; >, greater than]

\begin{tabular}{|c|c|c|c|c|c|c|c|c|c|c|c|c|c|}
\hline Date & Time & $\begin{array}{c}\text { Waterlevel } \\
\text { (feet below } \\
\text { measuring } \\
\text { point) }\end{array}$ & $\begin{array}{c}\text { Specific } \\
\text { conductance } \\
(\mu \mathrm{S} / \mathrm{cm})\end{array}$ & $\mathbf{p H}$ & $\begin{array}{c}\text { Temperature } \\
\left({ }^{\circ} \mathrm{C}\right)\end{array}$ & $\begin{array}{c}\text { Dissolved } \\
\text { oxygen } \\
\text { (mg/L) }\end{array}$ & $\begin{array}{c}\text { Hardness } \\
\text { (mg/L as } \\
\mathrm{CaCO}_{3} \text { ) }\end{array}$ & $\begin{array}{c}\text { Calcium } \\
\text { (mg/L) }\end{array}$ & $\begin{array}{l}\text { Magnesium } \\
(\mathrm{mg} / \mathrm{L})\end{array}$ & $\begin{array}{c}\text { Sodium } \\
\text { (mg/L) }\end{array}$ & $\begin{array}{l}\text { Potassium } \\
\text { (mg/L) }\end{array}$ & $\begin{array}{c}\text { Alkalinity, } \\
\text { fixed } \\
\text { endpoint } \\
\text { (mg/L) }\end{array}$ & $\begin{array}{c}\text { ANC } \\
\text { (mg/L) }\end{array}$ \\
\hline \multicolumn{14}{|c|}{ MW1-2A (measuring point altitude 573.38 feet above NGVD 29) } \\
\hline 06-03-99 & 0900 & 8.36 & 1,390 & 7.2 & 14.5 & $<0.1$ & 360 & 100 & 27.6 & 139 & 5.45 & -- & 344 \\
\hline 09-02-99 & 0810 & -- & 1,410 & 7.1 & 15.2 & $<.1$ & 400 & 112 & 28.9 & 148 & 5.92 & -- & 370 \\
\hline 03-07-00 & 1100 & 18.02 & 1,440 & 7.0 & 16.1 & $<.1$ & 390 & 108 & 30.4 & 140 & 5.69 & 364 & 365 \\
\hline $08-24-00$ & 1320 & 18.62 & 1,380 & 7.0 & 15.6 & $<.1$ & 390 & 108 & 30.2 & 125 & 5.46 & 356 & 359 \\
\hline 02-21-01 & 1310 & 19.38 & -- & 7.0 & 13.6 & $<.1$ & 410 & 113 & 31.3 & 131 & 4.95 & 364 & 364 \\
\hline 08-28-01 & 1440 & 14.43 & 1,430 & 7.1 & 16.2 & $<.1$ & 400 & 111 & 30.7 & 137 & 5.76 & 360 & 343 \\
\hline $02-28-02$ & 1310 & 18.51 & 1,700 & 7.2 & 14.7 & $<.1$ & 480 & 132 & 35.7 & 158 & 6.07 & 481 & 481 \\
\hline 08-20-02 & 1520 & 16.70 & 1,770 & 7.2 & 16.8 & $<.1$ & 480 & 135 & 35.3 & 175 & 6.16 & 414 & 382 \\
\hline 04-03-03 & 1000 & 20.82 & 1,560 & 7.1 & 15.4 & $<.1$ & -- & -- & -- & -- & -- & -- & -- \\
\hline $08-28-03$ & 1100 & 20.21 & 1,420 & 7.1 & 15.9 & $<.1$ & -- & -- & -- & -- & -- & -- & -- \\
\hline 02-26-04 & 1500 & 20.66 & 1,190 & 7.1 & 14.9 & $<.1$ & 470 & 131 & 35.3 & 85.1 & 5.49 & 333 & 181 \\
\hline $08-25-04$ & 1015 & 17.61 & 992 & 7.3 & 15.5 & -- & 450 & 125 & 33.5 & 46.5 & 4.86 & 414 & 230 \\
\hline $03-02-05$ & 1000 & 17.63 & 919 & 7.0 & 14.1 & -- & 490 & 137 & 35.7 & 27.7 & 4.20 & 430 & 422 \\
\hline 08-03-05 & 1100 & 18.71 & 989 & 7.0 & 15.0 & -- & 464 & 129 & 34.7 & 31.3 & 4.39 & 391 & 393 \\
\hline \multicolumn{14}{|c|}{ MW1-2B (measuring point altitude 573.45 feet above NGVD 29) } \\
\hline 06-03-99 & 0820 & 8.42 & 1,370 & 7.1 & 15.0 & $<.1$ & 540 & 154 & 38.1 & 60.4 & 5.87 & -- & 399 \\
\hline 09-02-99 & 0900 & -- & 1,400 & 7.1 & 15.2 & $<.1$ & 550 & 157 & 37.5 & 85.6 & 6.17 & -- & 388 \\
\hline $03-07-00$ & 1130 & 18.10 & 1,370 & 7.1 & 15.9 & $<.1$ & 450 & 127 & 31.7 & 90.8 & 5.99 & 379 & 380 \\
\hline $08-24-00$ & 1400 & 18.70 & 1,330 & 7.1 & 15.8 & $<.1$ & 470 & 135 & 33.0 & 73.8 & 6.41 & 399 & 400 \\
\hline 02-21-01 & 1350 & 19.46 & -- & 7.1 & 14.1 & $<.1$ & 470 & 136 & 32.6 & 109 & 5.97 & 380 & 379 \\
\hline 08-28-01 & 1530 & 14.48 & 1,410 & 7.2 & 15.9 & $<.1$ & 510 & 145 & 36.3 & 86.6 & 6.25 & 418 & 385 \\
\hline $02-28-02$ & 1400 & 18.98 & 1,470 & 7.3 & 14.5 & $<.1$ & 450 & 128 & 31.6 & 115 & 6.18 & 426 & 425 \\
\hline 08-20-02 & 1610 & 16.76 & 1,580 & 7.3 & 16.6 & $<.1$ & 510 & 144 & 35.6 & 117 & 6.38 & 432 & 361 \\
\hline $02-26-04$ & 1540 & 20.72 & 1,290 & 7.3 & 14.7 & -- & 480 & 137 & 32.6 & 99.5 & 6.55 & 325 & 387 \\
\hline $08-25-04$ & 1050 & 17.67 & 992 & 7.4 & 16.2 & -- & 420 & 119 & 29.0 & 50.7 & 5.67 & 421 & 321 \\
\hline $03-02-05$ & 1100 & 17.68 & 1,180 & 7.1 & 14.5 & -- & 500 & 142 & 35.6 & 66.3 & 6.13 & 433 & 438 \\
\hline 08-03-05 & 1115 & 18.75 & 1,400 & 7.2 & 14.9 & -- & 559 & 158 & 40.0 & 79.6 & 6.38 & 448 & 450 \\
\hline
\end{tabular}


$\left[\mu \mathrm{S} / \mathrm{cm}\right.$, microsiemens per centimeter at 25 degrees Celsius; $\mathrm{pH}$, in standard units; ${ }^{\circ} \mathrm{C}$, degrees Celsius; constituent concentrations are dissolved, unless otherwise noted; mg/L, milligrams per liter; $\mathrm{CaCO}_{3}$, calcium carbonate; ANC, total acid neutralizing capacity, incremental titration; N, nitrogen; $\mu \mathrm{g} / \mathrm{L}$, micrograms per liter; NGVD 29, National Geodetic Vertical Datum of 1929; --, no data;

$<$, less than; E, estimated; *, replicate sample; >, greater than]

\begin{tabular}{|c|c|c|c|c|c|c|c|c|c|c|c|c|c|}
\hline Date & Time & $\begin{array}{l}\text { Sulfate } \\
\text { (mg/L) }\end{array}$ & $\begin{array}{c}\text { Chloride } \\
\text { (mg/L) }\end{array}$ & $\begin{array}{c}\text { Fluoride } \\
\text { (mg/L) }\end{array}$ & $\begin{array}{c}\text { Residue, sum } \\
\text { of constituents } \\
(\mathrm{mg} / \mathrm{L}))\end{array}$ & $\begin{array}{c}\text { Nitrite as N } \\
(\mathrm{mg} / \mathrm{L})\end{array}$ & $\begin{array}{c}\text { Nitrite plus } \\
\text { nitrate as N } \\
\text { (mg/L) }\end{array}$ & $\begin{array}{c}\text { Ammonia as } \\
\mathbf{N} \\
(\mathrm{mg} / \mathrm{L})\end{array}$ & $\begin{array}{c}\text { Ammonia } \\
\text { plus organic } \\
\mathrm{N}, \text { total } \\
\text { (mg/L) }\end{array}$ & $\begin{array}{c}\text { Phosphorous, } \\
\text { total } \\
\text { (mg/L) }\end{array}$ & $\begin{array}{c}\text { Phosphorous, } \\
\text { dissolved } \\
\text { (mg/L) }\end{array}$ & $\begin{array}{c}\text { Ortho- } \\
\text { phosphorous } \\
\text { (mg/L) }\end{array}$ & $\begin{array}{c}\text { Iron } \\
(\mu \mathrm{g} / \mathrm{L})\end{array}$ \\
\hline \multicolumn{14}{|c|}{ MW1-2A } \\
\hline 06-03-99 & 0900 & 58.3 & 208 & 0.4 & 773 & $<0.010$ & $<0.05$ & 0.15 & 0.29 & 0.14 & $<0.05$ & 0.12 & 2,610 \\
\hline 09-02-99 & 0810 & 54.1 & 214 & .4 & 815 & $<.010$ & $<.05$ & .11 & .25 & .14 & .11 & .18 & 3,220 \\
\hline $03-07-00$ & 1100 & 69.0 & 232 & .3 & 806 & $<.010$ & $<.05$ & .11 & .26 & .11 & .09 & .07 & 2,470 \\
\hline $08-24-00$ & 1320 & 71.4 & 203 & .3 & 759 & $<.010$ & $<.05$ & .10 & .23 & .11 & .10 & .10 & 1,970 \\
\hline $02-21-01$ & 1310 & 66.7 & 204 & .4 & 772 & $<.006$ & $<.05$ & .08 & .20 & .12 & .06 & .04 & 1,500 \\
\hline 08-28-01 & 1440 & 62.0 & 207 & .3 & 773 & $<.006$ & $<.05$ & E.09 & .21 & .14 & .08 & E.11 & 2,990 \\
\hline $02-28-02$ & 1310 & 62.4 & 259 & .4 & 946 & E.004 & $<.05$ & .11 & .27 & .14 & .12 & .12 & 3,580 \\
\hline 08-20-02 & 1520 & 84.4 & 242 & .3 & 930 & $<.008$ & $<.05$ & .12 & .28 & .15 & .10 & .13 & 3,640 \\
\hline 04-03-03 & 1000 & -- & 221 & -- & -- & -- & -- & -- & -- & -- & -- & -- & -- \\
\hline $08-28-03$ & 1100 & -- & 198 & -- & -- & -- & -- & -- & -- & -- & -- & -- & -- \\
\hline $02-26-04$ & 1500 & 48.3 & 137 & .3 & 647 & E.050 & $<.60$ & .85 & .23 & .11 & .10 & $<.36$ & 2,650 \\
\hline $08-25-04$ & 1015 & 38.1 & 59.4 & .3 & 559 & $<.008$ & E.03 & .09 & .16 & .13 & .09 & .08 & 2,560 \\
\hline $03-02-05$ & 1000 & 32.4 & 36.2 & .3 & 528 & $<.008$ & $<.06$ & .06 & .13 & .06 & E.04 & E.13 & 1,490 \\
\hline 08-03-05 & 1100 & 35.9 & 78.2 & .3 & 552 & $<.008$ & $<.06$ & .08 & .15 & .10 & .04 & .02 & 2,700 \\
\hline \multicolumn{14}{|c|}{ MW1-2B } \\
\hline 06-03-99 & 0820 & 50.6 & 175 & .2 & 770 & .017 & $<.05$ & .73 & .48 & .21 & .32 & .27 & 10,200 \\
\hline 09-02-99 & 0900 & 64.7 & 198 & .2 & 829 & .013 & $<.05$ & .62 & .87 & .30 & .28 & .35 & 9,810 \\
\hline $03-07-00$ & 1130 & 57.3 & 194 & .2 & 745 & $<.010$ & $<.05$ & .57 & .77 & .29 & $<.05$ & .23 & 8,650 \\
\hline $08-24-00$ & 1400 & 37.0 & 173 & .2 & 708 & .014 & $<.05$ & .55 & .68 & .31 & .31 & .26 & 9,190 \\
\hline $02-21-01$ & 1350 & 59.9 & 215 & .3 & 796 & $<.006$ & $<.05$ & .59 & .78 & .33 & .36 & .09 & 9,220 \\
\hline 08-28-01 & 1530 & 36.5 & 186 & .2 & 756 & E.005 & $<.05$ & E.73 & .89 & .34 & .29 & E.27 & 8,800 \\
\hline $02-28-02$ & 1400 & 45.4 & 216 & .3 & 808 & .015 & $<.05$ & .70 & 1.1 & .33 & .25 & .30 & 8,440 \\
\hline 08-20-02 & 1610 & 44.9 & 208 & .2 & 826 & E.007 & $<.05$ & .69 & .87 & .34 & .33 & .38 & 8,930 \\
\hline $02-26-04$ & 1540 & 30.7 & 166 & .2 & 677 & $<.080$ & $<.60$ & .51 & .72 & .33 & .28 & E. 29 & 9,410 \\
\hline $08-25-04$ & 1050 & 1.70 & 72.2 & .2 & 539 & $<.008$ & $<.06$ & .52 & .63 & .35 & .25 & $<.02$ & 7,380 \\
\hline $03-02-05$ & 1100 & 13.7 & 123 & .2 & 659 & $<.008$ & $<.06$ & .51 & .68 & .30 & .16 & $<.02$ & 8,830 \\
\hline 08-03-05 & 1115 & 31.2 & 188 & .2 & 782 & $<.008$ & $<.06$ & .66 & .86 & .34 & .07 & $<.02$ & 8,320 \\
\hline
\end{tabular}


Table 1. Values of physical properties and concentrations of inorganic constituents and nutrients in samples from wells and surface-water sites, 1999-2005.-Continued

$\left[\mu \mathrm{S} / \mathrm{cm}\right.$, microsiemens per centimeter at 25 degrees Celsius; $\mathrm{pH}$, in standard units; ${ }^{\circ} \mathrm{C}$, degrees Celsius; constituent concentrations are dissolved, unless otherwise noted; mg/L, milligrams per liter; $\mathrm{CaCO}_{3}$, calcium carbonate; ANC, total acid neutralizing capacity, incremental titration; N, nitrogen; $\mu \mathrm{g} / \mathrm{L}$, micrograms per liter; NGVD 29, National Geodetic Vertical Datum of 1929; --, no data; $<$, less than; E, estimated; *, replicate sample; >, greater than]

\begin{tabular}{|c|c|c|c|c|c|c|c|c|c|c|c|c|c|}
\hline Date & Time & $\begin{array}{c}\text { Waterlevel } \\
\text { (feet below } \\
\text { measuring } \\
\text { point) }\end{array}$ & $\begin{array}{c}\text { Specific } \\
\text { conductance } \\
(\mu \mathrm{S} / \mathrm{cm})\end{array}$ & pH & $\begin{array}{c}\text { Temperature } \\
\left({ }^{\circ} \mathbf{C}\right)\end{array}$ & $\begin{array}{c}\text { Dissolved } \\
\text { oxygen } \\
\text { (mg/L) }\end{array}$ & $\begin{array}{c}\text { Hardness } \\
(\mathrm{mg} / \mathrm{L} \text { as } \\
\left.\mathrm{CaCO}_{3}\right)\end{array}$ & $\begin{array}{c}\text { Calcium } \\
\text { (mg/L) }\end{array}$ & $\begin{array}{l}\text { Magnesium } \\
(\mathrm{mg} / \mathrm{L})\end{array}$ & $\begin{array}{c}\text { Sodium } \\
\text { (mg/L) }\end{array}$ & $\begin{array}{l}\text { Potassium } \\
\text { (mg/L) }\end{array}$ & $\begin{array}{c}\text { Alkalinity, } \\
\text { fixed } \\
\text { endpoint } \\
\text { (mg/L) }\end{array}$ & $\begin{array}{c}\text { ANC } \\
\text { (mg/L) }\end{array}$ \\
\hline \multicolumn{14}{|c|}{ MW1-3A (measuring point altitude 575.05 feet above NGVD 29) } \\
\hline 06-03-99 & 1250 & 10.42 & 783 & 7.1 & 15.3 & $<0.1$ & 400 & 104 & 32.6 & 5.54 & 4.90 & -- & 340 \\
\hline 09-01-99 & 1440 & -- & 971 & 7.2 & 15.2 & $<.1$ & 480 & 126 & 41.0 & 6.18 & 4.60 & -- & 335 \\
\hline $03-07-00$ & 1440 & 20.26 & 1,040 & 7.2 & 16.8 & $<.1$ & 490 & 129 & 41.8 & 6.07 & 5.18 & 336 & 337 \\
\hline $08-22-00$ & 1230 & 21.34 & 1,120 & 7.1 & 16.9 & $<.1$ & 550 & 142 & 47.0 & 6.82 & 4.76 & 349 & 354 \\
\hline 02-20-01 & 1640 & 21.75 & 1,120 & 7.0 & 14.6 & $<.1$ & 580 & 153 & 48.8 & 8.76 & 4.88 & 360 & 359 \\
\hline $08-28-01$ & 1610 & 16.39 & 1,300 & 7.1 & 15.8 & $<.1$ & 640 & 169 & 52.3 & 1.08 & 4.71 & 340 & 289 \\
\hline $02-28-02$ & 1520 & 20.84 & 1,170 & 7.2 & 15.0 & $<.1$ & 540 & 144 & 45.0 & 13.2 & 5.55 & 364 & 363 \\
\hline 08-20-02 & 0930 & 19.37 & 1,300 & 7.2 & 15.8 & $<.1$ & 590 & 158 & 47.8 & 18.0 & 4.96 & 361 & 293 \\
\hline 04-02-03 & 1630 & 23.50 & 1,140 & 7.2 & 16.3 & $<.1$ & -- & -- & -- & -- & -- & -- & -- \\
\hline $08-28-03$ & 1140 & 22.51 & 1,230 & 7.1 & 16.4 & $<.1$ & -- & -- & -- & -- & -- & -- & -- \\
\hline $02-27-04$ & 1000 & 23.17 & 1,220 & 7.1 & 15.0 & -- & 600 & 159 & 49.6 & 33.1 & 6.21 & 246 & 211 \\
\hline $08-25-04$ & 1430 & 19.60 & 1,380 & 7.2 & 16.8 & -- & 630 & 167 & 51.3 & 42.3 & 5.79 & 388 & 239 \\
\hline 03-07-05 & 1100 & 19.81 & 1,320 & 7.0 & 14.8 & -- & 590 & 154 & 50.3 & 48.3 & 6.27 & 384 & 446 \\
\hline 08-03-05 & 1525 & 20.67 & 1,255 & 7.1 & 15.0 & -- & 576 & 154 & 46.3 & 48.4 & 5.77 & 385 & 390 \\
\hline \multicolumn{14}{|c|}{ MW1-3B (measuring point altitude 574.97 feet above NGVD 29) } \\
\hline 06-03-99 & 1350 & 10.45 & 915 & 7.1 & 15.2 & $<.1$ & 450 & 128 & 31.8 & 5.98 & 3.61 & -- & 337 \\
\hline 09-01-99 & 1550 & -- & 1,030 & 7.1 & 15.2 & $<.1$ & 520 & 146 & 37.5 & 6.89 & 4.01 & -- & 362 \\
\hline $03-07-00$ & 1410 & 20.30 & 1,090 & 7.1 & 15.8 & $<.1$ & 510 & 145 & 36.8 & 8.66 & 4.19 & 367 & 367 \\
\hline $08-22-00$ & 1310 & 21.39 & 1,150 & 7.0 & 16.7 & $<.1$ & 540 & 152 & 39.1 & 12.6 & 4.34 & 372 & 378 \\
\hline 02-20-01 & 1600 & 21.78 & 1,040 & 7.0 & 14.2 & $<.1$ & 530 & 152 & 37.5 & 11.8 & 3.88 & 358 & 359 \\
\hline 08-28-01 & 1645 & 16.44 & 1,210 & 7.1 & 15.8 & $<.1$ & 580 & 165 & 40.3 & 13.8 & 4.53 & 375 & 293 \\
\hline $02-28-02$ & 1440 & 20.89 & 1,210 & 7.2 & 14.5 & $<.1$ & 540 & 155 & 38.0 & 15.1 & 4.57 & 374 & 374 \\
\hline $08-20-02$ & 0830 & 19.40 & 1,330 & 7.2 & 15.8 & $<.1$ & 590 & 170 & 40.1 & 18.8 & 4.59 & 384 & 301 \\
\hline $02-27-04$ & 0920 & 23.21 & 1,260 & 7.1 & 14.8 & $<.1$ & 620 & 177 & 42.7 & 31.2 & 4.97 & 273 & 173 \\
\hline $08-25-04$ & 1345 & 19.64 & 1,340 & 7.3 & 15.8 & -- & 630 & 180 & 42.7 & 35.8 & 4.75 & 379 & 157 \\
\hline $03-07-05$ & 1140 & 19.85 & 1,320 & 7.1 & 14.7 & -- & 610 & 175 & 41.4 & 39.4 & 4.89 & 389 & 450 \\
\hline$*$ & 1150 & 19.85 & 1,320 & 7.1 & 14.7 & -- & 600 & 173 & 40.9 & 38.8 & 4.95 & 389 & 450 \\
\hline 08-03-05 & 1520 & 20.71 & 1,295 & 7.2 & 15.0 & -- & 605 & 174 & 41.4 & 39.2 & 4.79 & 408 & 416 \\
\hline
\end{tabular}


$\left[\mu \mathrm{S} / \mathrm{cm}\right.$, microsiemens per centimeter at 25 degrees Celsius; $\mathrm{pH}$, in standard units; ${ }^{\circ} \mathrm{C}$, degrees Celsius; constituent concentrations are dissolved, unless otherwise noted; mg/L, milligrams per liter; $\mathrm{CaCO}_{3}$, calcium carbonate; ANC, total acid neutralizing capacity, incremental titration; N, nitrogen; $\mu \mathrm{g} / \mathrm{L}$, micrograms per liter; NGVD 29, National Geodetic Vertical Datum of 1929; --, no data; $<$, less than; E, estimated; *, replicate sample; >, greater than]

\begin{tabular}{|c|c|c|c|c|c|c|c|c|c|c|c|c|c|}
\hline Date & Time & $\begin{array}{l}\text { Sulfate } \\
\text { (mg/L) }\end{array}$ & $\begin{array}{c}\text { Chloride } \\
\text { (mg/L) }\end{array}$ & $\begin{array}{c}\text { Fluoride } \\
\text { (mg/L) }\end{array}$ & $\begin{array}{l}\text { Residue, sum } \\
\text { of constituents } \\
\text { (mg/L)) }\end{array}$ & $\begin{array}{c}\text { Nitrite as N } \\
(\mathbf{m g} / \mathbf{L})\end{array}$ & $\begin{array}{c}\text { Nitrite plus } \\
\text { nitrate as N } \\
\text { (mg/L) }\end{array}$ & $\begin{array}{c}\text { Ammonia as } \\
\mathbf{N} \\
(\mathrm{mg} / \mathrm{L})\end{array}$ & $\begin{array}{c}\text { Ammonia } \\
\text { plus organic } \\
\mathrm{N}, \text { total } \\
(\mathrm{mg} / \mathrm{L})\end{array}$ & $\begin{array}{c}\text { Phosphorous, } \\
\text { total } \\
\text { (mg/L) }\end{array}$ & $\begin{array}{c}\text { Phosphorous, } \\
\text { dissolved } \\
\text { (mg/L) }\end{array}$ & $\begin{array}{c}\text { Ortho- } \\
\text { phosphorous } \\
\text { (mg/L) }\end{array}$ & $\begin{array}{l}\text { Iron } \\
(\mu \mathrm{g} / \mathrm{L})\end{array}$ \\
\hline \multicolumn{14}{|c|}{ MW1-3A } \\
\hline 06-03-99 & 1250 & 35.5 & 34.7 & 0.3 & 475 & 0.012 & $<0.05$ & 0.66 & 0.70 & 0.64 & 0.28 & 0.36 & 7,100 \\
\hline 09-01-99 & 1440 & 51.1 & 92.6 & .3 & 574 & $<.010$ & $<.05$ & .43 & .43 & .44 & .35 & .54 & 7,190 \\
\hline $03-07-00$ & 1440 & 57.0 & 114 & .3 & 563 & $<.010$ & $<.05$ & .47 & .53 & .37 & $<.05$ & .23 & 7,080 \\
\hline $08-22-00$ & 1230 & 53.3 & 131 & .3 & 602 & .018 & $<.05$ & .43 & .54 & .30 & .18 & .12 & 6,920 \\
\hline 02-20-01 & 1640 & 63.9 & 147 & .3 & 647 & $<.006$ & $<.05$ & .38 & .44 & .21 & .12 & .05 & 4,250 \\
\hline 08-28-01 & 1610 & 69.7 & 166 & .3 & 683 & $<.006$ & $<.05$ & E.29 & .39 & .21 & .14 & E.03 & 5,980 \\
\hline $02-28-02$ & 1520 & 64.1 & 143 & .3 & 642 & .016 & $<.05$ & .49 & .57 & .28 & .24 & .24 & 6,850 \\
\hline $08-20-02$ & 0930 & 64.6 & 147 & .3 & 663 & E.004 & $<.05$ & .38 & .47 & .23 & .17 & .13 & 6,420 \\
\hline $04-02-03$ & 1630 & -- & 128 & -- & -- & -- & -- & -- & -- & -- & -- & -- & -- \\
\hline $08-28-03$ & 1140 & -- & 167 & -- & -- & -- & -- & -- & -- & -- & -- & -- & -- \\
\hline $02-27-04$ & 1000 & 67.4 & 161 & .3 & 624 & $<.080$ & $<.60$ & $<.40$ & .30 & .04 & $<.04$ & $<.36$ & -- \\
\hline $08-25-04$ & 1430 & 75.2 & 174 & .3 & 753 & $<.008$ & $<.06$ & .52 & .63 & .16 & .12 & $<.02$ & 4,010 \\
\hline $03-07-05$ & 1100 & 74.1 & 165 & .4 & 773 & $<.008$ & $<.06$ & .48 & .63 & .16 & .10 & .07 & 5,870 \\
\hline 08-03-05 & 1525 & 72.1 & 156 & .3 & 724 & $<.008$ & $<.06$ & .48 & .57 & .16 & .05 & .03 & 6,000 \\
\hline \multicolumn{14}{|c|}{ MW1-3B } \\
\hline 06-03-99 & 1350 & 32.3 & 73.7 & .3 & 521 & $<.010$ & $<0.05$ & 0.41 & 0.40 & 0.32 & 0.39 & 0.29 & 6,150 \\
\hline 09-01-99 & 1550 & 39.1 & 107 & .3 & 601 & $<.010$ & $<.05$ & .33 & .34 & .27 & .23 & .32 & 6,970 \\
\hline $03-07-00$ & 1410 & 41.5 & 120 & .3 & 585 & $<.010$ & $<.05$ & .34 & .42 & .28 & $<.05$ & .22 & 6,680 \\
\hline $08-22-00$ & 1310 & 37.3 & 131 & .3 & 608 & $<.010$ & $<.05$ & .35 & .47 & .31 & .14 & .06 & 7,170 \\
\hline 02-20-01 & 1600 & 43.4 & 123 & .4 & 595 & $<.006$ & $<.05$ & .34 & .41 & .30 & .17 & .07 & 7,230 \\
\hline $08-28-01$ & 1645 & 48.5 & 140 & .3 & 644 & E.009 & $<.05$ & E.39 & .48 & .31 & .26 & E. 22 & 7,490 \\
\hline $02-28-02$ & 1440 & 46.4 & 148 & .4 & 640 & .013 & $<.05$ & .38 & .51 & .29 & .26 & .15 & 7,060 \\
\hline 08-20-02 & 0830 & 44.8 & 161 & .3 & 678 & .011 & $<.05$ & .41 & .51 & .29 & .30 & .29 & 7,500 \\
\hline $02-27-04$ & 0920 & 44.6 & 175 & .4 & 648 & E.045 & $<.60$ & E.36 & .56 & .31 & .22 & E.27 & 8,450 \\
\hline $08-25-04$ & 1345 & 48.0 & 175 & .3 & 723 & $<.008$ & $<.06$ & .43 & .54 & .29 & .22 & $<.02$ & 8,130 \\
\hline $03-07-05$ & 1140 & 45.9 & 174 & .4 & 759 & $<.008$ & $<.06$ & .41 & .53 & .29 & .23 & $<.02$ & 7,930 \\
\hline$*$ & 1150 & 46.1 & 173 & .4 & 755 & $<.008$ & $<.06$ & .41 & .52 & .28 & .21 & $<.02$ & 7,720 \\
\hline 08-03-05 & 1520 & 43.7 & 175 & .4 & 736 & $<.008$ & $<.06$ & .41 & .56 & .30 & .14 & .06 & 7,160 \\
\hline
\end{tabular}


Table 1. Values of physical properties and concentrations of inorganic constituents and nutrients in samples from wells and surface-water sites, 1999-2005.-Continued

$\left[\mu \mathrm{S} / \mathrm{cm}\right.$, microsiemens per centimeter at 25 degrees Celsius; $\mathrm{pH}$, in standard units; ${ }^{\circ} \mathrm{C}$, degrees Celsius; constituent concentrations are dissolved, unless otherwise noted; mg/L, milligrams per liter; $\mathrm{CaCO}_{3}$, calcium carbonate; ANC, total acid neutralizing capacity, incremental titration; N, nitrogen; $\mu \mathrm{g} / \mathrm{L}$, micrograms per liter; NGVD 29, National Geodetic Vertical Datum of 1929; --, no data; $<$, less than; E, estimated; *, replicate sample; >, greater than]

\begin{tabular}{|c|c|c|c|c|c|c|c|c|c|c|c|c|c|}
\hline Date & Time & $\begin{array}{c}\text { Waterlevel } \\
\text { (feet below } \\
\text { measuring } \\
\text { point) }\end{array}$ & $\begin{array}{c}\text { Specific } \\
\text { conductance } \\
(\mu \mathrm{S} / \mathrm{cm})\end{array}$ & $\mathbf{p H}$ & $\begin{array}{c}\text { Temperature } \\
\left({ }^{\circ} \mathrm{C}\right)\end{array}$ & $\begin{array}{c}\text { Dissolved } \\
\text { oxygen } \\
\text { (mg/L) }\end{array}$ & $\begin{array}{c}\text { Hardness } \\
\text { (mg/L as } \\
\mathrm{CaCO}_{3} \text { ) }\end{array}$ & $\begin{array}{c}\text { Calcium } \\
\text { (mg/L) }\end{array}$ & $\begin{array}{l}\text { Magnesium } \\
\quad(\mathrm{mg} / \mathrm{L})\end{array}$ & $\begin{array}{l}\text { Sodium } \\
\text { (mg/L) }\end{array}$ & $\begin{array}{l}\text { Potassium } \\
\text { (mg/L) }\end{array}$ & $\begin{array}{l}\text { Alkalinity, } \\
\text { fixed } \\
\text { endpoint } \\
\text { (mg/L) }\end{array}$ & $\begin{array}{c}\text { ANC } \\
\text { (mg/L) }\end{array}$ \\
\hline \multicolumn{14}{|c|}{ MW1-4A (measuring point altitude 583.46 feet above NGVD 29) } \\
\hline 06-01-99 & 1530 & 11.16 & 855 & 6.8 & 15.2 & $<0.1$ & 420 & 120 & 27.9 & 18.3 & 1.84 & -- & 405 \\
\hline * & 1535 & 11.16 & 855 & 6.8 & 15.2 & $<.1$ & 440 & 129 & 29.0 & 18.7 & 1.75 & -- & 405 \\
\hline 09-02-99 & 1410 & -- & 838 & 7.0 & 14.9 & $<.1$ & 470 & 137 & 30.0 & 19.4 & 1.80 & -- & 412 \\
\hline * & 1415 & -- & 838 & 7.0 & 14.9 & $<.1$ & 470 & 138 & 30.1 & 19.1 & 1.84 & -- & 413 \\
\hline $03-02-00$ & 0820 & 12.49 & 775 & 6.6 & 13.8 & $<.1$ & 390 & 112 & 26.1 & 17.7 & 1.70 & 397 & 399 \\
\hline$*$ & 0825 & 12.49 & 775 & 6.6 & 13.8 & $<.1$ & 390 & 112 & 26.0 & 17.6 & 1.74 & 397 & 399 \\
\hline $08-22-00$ & 1420 & 12.83 & 758 & 6.9 & 16.9 & $<.1$ & 370 & 107 & 25.1 & 17.8 & 1.60 & 367 & 385 \\
\hline * & 1425 & 12.83 & 758 & 6.9 & 16.9 & $<.1$ & 370 & 108 & 24.7 & 16.7 & 1.78 & 381 & 376 \\
\hline 02-21-01 & 0820 & 11.52 & 756 & 6.9 & 13.5 & $<.1$ & 400 & 116 & 26.2 & 18.2 & 1.60 & 371 & 370 \\
\hline * & 0825 & 11.52 & 756 & 6.9 & 13.5 & $<.1$ & -- & 117 & 26.5 & 18.4 & 1.59 & 370 & 370 \\
\hline 08-28-01 & 1140 & 11.83 & 807 & 7.0 & 16.1 & $<.1$ & 420 & 121 & 27.5 & 19.1 & 1.74 & 373 & 366 \\
\hline$*$ & 1145 & 11.83 & 807 & 7.0 & 16.1 & $<.1$ & -- & 120 & 27.4 & 19.6 & 1.69 & 365 & 342 \\
\hline $02-28-02$ & 0920 & 11.72 & 718 & 7.0 & 14.3 & $<.1$ & 360 & 106 & 23.1 & 17.1 & 1.62 & 369 & 366 \\
\hline * & 0925 & 11.72 & 718 & 7.0 & 14.3 & $<.1$ & -- & -- & -- & -- & -- & -- & -- \\
\hline 08-20-02 & 1130 & 11.91 & 729 & 7.1 & 16.6 & $<.1$ & 350 & 102 & 22.4 & 17.9 & 1.70 & 341 & 301 \\
\hline$*$ & 1135 & 11.91 & 729 & 7.1 & 16.6 & $<.1$ & -- & -- & -- & -- & -- & -- & -- \\
\hline $04-02-03$ & 1530 & 11.35 & 731 & 7.0 & 15.5 & $<.1$ & -- & -- & -- & -- & -- & -- & -- \\
\hline $08-28-03$ & 0920 & 13.22 & 745 & 7.0 & 15.7 & $<.1$ & -- & -- & -- & -- & -- & -- & -- \\
\hline 02-26-04 & 1030 & 11.28 & 787 & 7.0 & 14.4 & -- & 420 & 124 & 27.4 & 19.8 & 1.90 & 276 & 179 \\
\hline$*$ & 1035 & 11.28 & 787 & 7.0 & 14.4 & -- & -- & 118 & 26.9 & 19.4 & 1.7 & 293 & 232 \\
\hline 08-24-04 & 1130 & 12.14 & 827 & 7.2 & 15.2 & -- & 460 & 135 & 29.4 & 20.3 & 1.88 & 383 & 220 \\
\hline $03-02-05$ & 1345 & 10.57 & 810 & 6.8 & 15.1 & -- & 450 & 132 & 29.1 & 17.9 & 1.81 & 394 & 387 \\
\hline $08-03-05$ & 1400 & 12.62 & 810 & 7.0 & 14.6 & -- & 424 & 125 & 27.0 & 17.7 & 1.60 & 378 & 383 \\
\hline \multicolumn{14}{|c|}{ MW1-4B (measuring point altitude 583.53 feet above NGVD 29) } \\
\hline 06-01-99 & 1430 & 15.92 & 712 & 6.8 & 15.9 & $<.1$ & 330 & 94.1 & 22.1 & 17.0 & 2.92 & -- & 362 \\
\hline 09-02-99 & 1320 & -- & 610 & 7.2 & 15.6 & $<.1$ & 290 & 85.2 & 18.3 & 14.9 & 4.18 & -- & 340 \\
\hline $03-02-00$ & 0910 & 23.46 & 569 & 6.8 & 14.5 & $<.1$ & 260 & 77.1 & 16.7 & 13.9 & 3.99 & 328 & 331 \\
\hline $08-22-00$ & 0510 & 23.29 & 595 & 7.1 & 18.1 & $<.1$ & 270 & 79.3 & 16.7 & 12.5 & 4.16 & 329 & 329 \\
\hline 02-21-01 & 0930 & 21.26 & 566 & 7.2 & 13.4 & $<.1$ & 270 & 80.5 & 17.2 & 14.1 & 3.70 & 326 & 325 \\
\hline 08-28-01 & 1220 & 21.69 & 620 & 7.2 & 15.0 & $<.1$ & 290 & 84.2 & 18.5 & 15.0 & 3.76 & 331 & 331 \\
\hline
\end{tabular}


$\left[\mu \mathrm{S} / \mathrm{cm}\right.$, microsiemens per centimeter at 25 degrees Celsius; $\mathrm{pH}$, in standard units; ${ }^{\circ} \mathrm{C}$, degrees Celsius; constituent concentrations are dissolved, unless otherwise noted; mg/L, milligrams per liter; $\mathrm{CaCO}_{3}$, calcium carbonate; ANC, total acid neutralizing capacity, incremental titration; N, nitrogen; $\mu \mathrm{g} / \mathrm{L}$, micrograms per liter; NGVD 29, National Geodetic Vertical Datum of 1929; --, no data; $<$, less than; E, estimated; *, replicate sample; >, greater than]

\begin{tabular}{|c|c|c|c|c|c|c|c|c|c|c|c|c|c|}
\hline Date & Time & $\begin{array}{l}\text { Sulfate } \\
\text { (mg/L) }\end{array}$ & $\begin{array}{c}\text { Chloride } \\
\text { (mg/L) }\end{array}$ & $\begin{array}{c}\text { Fluoride } \\
\text { (mg/L) }\end{array}$ & $\begin{array}{c}\text { Residue, sum } \\
\text { of } \\
\text { constituents } \\
(\mathbf{m g} / \mathrm{L}))\end{array}$ & $\begin{array}{c}\text { Nitrite as N } \\
\quad(\mathbf{m g} / \mathbf{L})\end{array}$ & $\begin{array}{c}\text { Nitrite plus } \\
\text { nitrate as N } \\
\quad(\mathrm{mg} / \mathrm{L})\end{array}$ & $\underset{\substack{\mathbf{N} \\
(\mathbf{m g} / \mathrm{L})}}{\text { Ammonia as }}$ & $\begin{array}{c}\text { Ammonia } \\
\text { plus organic } \\
\text { N, total } \\
\text { (mg/L) }\end{array}$ & $\begin{array}{c}\text { Phosphorous, } \\
\text { total } \\
\text { (mg/L) }\end{array}$ & $\begin{array}{l}\text { Phosphorous, } \\
\text { dissolved } \\
(\mathrm{mg} / \mathrm{L})\end{array}$ & $\begin{array}{c}\text { Ortho- } \\
\text { phosphorous } \\
\text { (mg/L) }\end{array}$ & $\begin{array}{c}\text { Iron } \\
(\mu \mathrm{g} / \mathrm{L})\end{array}$ \\
\hline \multicolumn{14}{|c|}{ MW1-4A } \\
\hline 06-01-99 & 1530 & 72.8 & 7.92 & 0.4 & 510 & $<0.010$ & $<0.05$ & 0.17 & 0.18 & 0.10 & E0.03 & 0.09 & 103 \\
\hline$*$ & 1535 & 72.9 & 7.13 & .4 & 520 & $<.010$ & $<.05$ & .16 & .18 & .10 & .06 & .09 & 114 \\
\hline 09-02-99 & 1410 & 73.4 & 8.92 & .4 & 538 & $<.010$ & $<.05$ & .10 & .15 & .09 & .07 & .07 & 142 \\
\hline$*$ & 1415 & 73.8 & 8.80 & .4 & 540 & $<.010$ & $<.05$ & .12 & .16 & .09 & .08 & .09 & 165 \\
\hline $03-02-00$ & 0820 & 68.5 & 6.21 & .4 & 471 & $<.010$ & $<.05$ & .10 & .16 & .08 & .07 & .06 & 76 \\
\hline$*$ & 0825 & 68.2 & 5.42 & .4 & 469 & $<.010$ & $<.05$ & .10 & .18 & .07 & .08 & .07 & 74 \\
\hline $08-22-00$ & 1420 & 53.4 & 6.56 & .4 & 432 & $<.010$ & $<.05$ & .09 & .17 & .09 & .09 & .07 & 82 \\
\hline * & 1425 & 53.1 & 6.57 & .3 & 440 & $<.010$ & $<.05$ & .09 & .16 & .10 & .09 & .07 & 56 \\
\hline $02-21-01$ & 0820 & 55.8 & 16.6 & .4 & 458 & $<.006$ & $<.05$ & .09 & .15 & .09 & .09 & .08 & 94 \\
\hline$*$ & 0825 & 55.7 & 16.6 & .4 & -- & $<.006$ & $<.05$ & .08 & .13 & .10 & .08 & .08 & 25 \\
\hline 08-28-01 & 1140 & 53.8 & 15.3 & .4 & 463 & $<.006$ & $<.05$ & E.11 & .16 & .10 & .06 & E.05 & 181 \\
\hline$*$ & 1145 & 51.7 & 15.2 & .4 & -- & $<.006$ & $<.05$ & E.09 & .13 & .08 & .07 & E.06 & 81 \\
\hline $02-28-02$ & 0920 & 39.8 & 8.14 & .4 & 418 & $<.008$ & $<.05$ & .08 & .15 & .10 & .08 & .07 & 86 \\
\hline$*$ & 0925 & -- & -- & -- & -- & -- & $<.05$ & $<.21$ & .14 & .09 & .09 & .08 & -- \\
\hline 08-20-02 & 1130 & 32.2 & 7.11 & .4 & 388 & $<.008$ & $<.05$ & .09 & .13 & .10 & .08 & .07 & 74 \\
\hline * & 1135 & -- & -- & -- & -- & -- & -- & -- & -- & -- & -- & -- & -- \\
\hline $04-02-03$ & 1530 & -- & 5.95 & -- & -- & -- & -- & -- & -- & -- & -- & -- & -- \\
\hline $08-28-03$ & 0920 & -- & 11.4 & -- & -- & -- & -- & -- & -- & -- & -- & -- & -- \\
\hline $02-26-04$ & 1030 & 47.7 & 13.9 & .4 & 401 & E.004 & $<.06$ & .08 & .15 & .09 & .08 & E.09 & 44 \\
\hline$*$ & 1035 & 50.1 & 14.1 & .4 & -- & E.004 & $<.06$ & .08 & .15 & .09 & .08 & $<.18$ & 58 \\
\hline 08-24-04 & 1130 & 53.9 & 14.6 & .4 & 486 & $<.008$ & $<.06$ & .09 & .17 & .12 & .06 & .05 & 115 \\
\hline $03-02-05$ & 1345 & 54.4 & 8.8 & .4 & 477 & $<.008$ & $<.06$ & .09 & .16 & .09 & .06 & .05 & 118 \\
\hline 08-03-05 & 1400 & 56.5 & 7.01 & .4 & 466 & $<.008$ & $<.06$ & .09 & .15 & .10 & .08 & .04 & 162 \\
\hline \multicolumn{14}{|c|}{ MW1-4B } \\
\hline 06-01-99 & 1430 & 28.2 & 4.95 & .3 & 424 & $<.010$ & $<.05$ & 2.33 & 2.1 & .67 & $<.05$ & .39 & 3,840 \\
\hline 09-02-99 & 1320 & .3 & 3.65 & .2 & 384 & $<.010$ & $<.05$ & 4.03 & 3.4 & 1.20 & .72 & 1.83 & 6,890 \\
\hline $03-02-00$ & 0910 & $<.3$ & 2.60 & .2 & -- & $<.010$ & $<.05$ & 3.63 & 4.0 & 1.33 & .39 & 1.25 & 6,840 \\
\hline $08-22-00$ & 0510 & $<.3$ & 3.03 & .2 & -- & $<.010$ & $<.05$ & 3.50 & 4.1 & 1.33 & 1.45 & 1.12 & 7,060 \\
\hline $02-21-01$ & 0930 & E. 1 & 2.54 & .2 & -- & $<.006$ & $<.05$ & 3.50 & 3.8 & 1.23 & .98 & .63 & 7,260 \\
\hline 08-28-01 & 1220 & .6 & 3.17 & .2 & 330 & $<.006$ & $<.05$ & E3.52 & 3.7 & 1.28 & .90 & E.71 & 6,420 \\
\hline
\end{tabular}


Table 1. Values of physical properties and concentrations of inorganic constituents and nutrients in samples from wells and surface-water sites, 1999-2005.-Continued

$\left[\mu \mathrm{S} / \mathrm{cm}\right.$, microsiemens per centimeter at 25 degrees Celsius; $\mathrm{pH}$, in standard units; ${ }^{\circ} \mathrm{C}$, degrees Celsius; constituent concentrations are dissolved, unless otherwise noted; mg/L, milligrams per liter; $\mathrm{CaCO}_{3}$, calcium carbonate; ANC, total acid neutralizing capacity, incremental titration; N, nitrogen; $\mu \mathrm{g} / \mathrm{L}$, micrograms per liter; NGVD 29, National Geodetic Vertical Datum of 1929; --, no data; $<$, less than; E, estimated; *, replicate sample; >, greater than]

\begin{tabular}{|c|c|c|c|c|c|c|c|c|c|c|c|c|c|}
\hline Date & Time & $\begin{array}{l}\text { Waterlevel } \\
\text { (feet below } \\
\text { measuring } \\
\text { point) }\end{array}$ & $\begin{array}{c}\text { Specific } \\
\text { conductance } \\
(\mu \mathrm{S} / \mathrm{cm})\end{array}$ & pH & $\begin{array}{c}\text { Temperature } \\
\left({ }^{\circ} \mathbf{C}\right)\end{array}$ & $\begin{array}{c}\text { Dissolved } \\
\text { oxygen } \\
\text { (mg/L) }\end{array}$ & $\begin{array}{c}\text { Hardness } \\
\text { (mg/L as } \\
\mathrm{CaCO}_{3} \text { ) }\end{array}$ & $\begin{array}{c}\text { Calcium } \\
\text { (mg/L) }\end{array}$ & $\begin{array}{l}\text { Magnesium } \\
(\mathrm{mg} / \mathrm{L})\end{array}$ & $\begin{array}{l}\text { Sodium } \\
\text { (mg/L) }\end{array}$ & $\begin{array}{l}\text { Potassium } \\
\text { (mg/L) }\end{array}$ & $\begin{array}{c}\text { Alkalinity, } \\
\text { fixed } \\
\text { endpoint } \\
\text { (mg/L) }\end{array}$ & $\begin{array}{l}\text { ANC } \\
\text { (mg/L) }\end{array}$ \\
\hline \multicolumn{14}{|c|}{ MW1-4B (measuring point altitude 583.53 feet above NGVD 29)—Continued } \\
\hline $02-28-02$ & 0830 & 22.77 & 596 & 7.1 & 14.1 & $<0.1$ & 270 & 81.2 & 17.0 & 13.4 & 3.73 & 327 & 326 \\
\hline $08-20-02$ & 1030 & 21.98 & 644 & 7.2 & 16.6 & $<.1$ & 280 & 83.6 & 17.5 & 14.0 & 3.79 & 334 & 326 \\
\hline $02-26-04$ & 1120 & 21.87 & 580 & 7.2 & 14.4 & $<.1$ & 290 & 87.4 & 18.1 & 14.9 & 3.98 & 329 & 311 \\
\hline 08-24-04 & 1230 & 22.25 & 610 & 7.3 & 16.3 & -- & 300 & 89.5 & 18.3 & 15.0 & 4.04 & 329 & 303 \\
\hline 03-02-05 & 1415 & 20.10 & 699 & 7.1 & 14.9 & -- & 390 & 112 & 26.0 & 17.8 & 2.17 & 356 & 369 \\
\hline 08-03-05 & 1410 & 23.11 & 587 & 7.1 & 14.8 & -- & 296 & 88.6 & 18.1 & 15.1 & 3.46 & 331 & 340 \\
\hline \multicolumn{14}{|c|}{ MW2-1A (measuring point altitude 576.89 feet above NGVD 29) } \\
\hline 06-02-99 & 1300 & 12.64 & 821 & 6.9 & 15.1 & $<.1$ & 440 & 130 & 28.4 & 5.71 & 3.15 & -- & 454 \\
\hline 08-27-99 & 0810 & 18.88 & 744 & 6.7 & 14.7 & $<.1$ & 430 & 125 & 28.2 & 4.56 & 2.36 & -- & 412 \\
\hline 02-29-00 & 1520 & 23.38 & 664 & 6.9 & 15.8 & $<.1$ & 380 & 111 & 25.6 & 4.26 & 2.34 & 411 & 411 \\
\hline $08-29-00$ & 1320 & 22.28 & 787 & 6.9 & 16.2 & $<.1$ & 440 & 129 & 29.2 & 5.69 & 2.88 & 446 & 449 \\
\hline 03-01-01 & 0930 & 21.19 & 770 & 7.0 & 14.6 & $<.1$ & 410 & 120 & 25.5 & 5.97 & 3.29 & 443 & 453 \\
\hline 08-23-01 & 0830 & 21.52 & 797 & 6.9 & 14.3 & $<.1$ & 440 & 128 & 28.7 & 5.15 & 2.86 & 365 & 349 \\
\hline $02-27-02$ & 0910 & 24.17 & 740 & 7.0 & 15.0 & $<.1$ & 400 & 117 & 25.5 & 4.43 & 2.72 & 417 & 418 \\
\hline $08-22-02$ & 0900 & 23.14 & 757 & 7.0 & 15.7 & $<.1$ & 400 & 120 & 25.7 & 4.95 & 2.55 & 371 & 279 \\
\hline $02-26-04$ & 0920 & 24.83 & 767 & 7.0 & 15.1 & -- & 460 & 135 & 28.8 & 5.38 & 3.05 & 287 & 216 \\
\hline $08-25-04$ & 1535 & 22.45 & 798 & 7.0 & 16.3 & -- & 460 & 136 & 29.2 & 5.29 & 3.11 & 370 & 274 \\
\hline 03-04-05 & 1245 & 22.39 & 702 & 6.9 & 15.5 & -- & 400 & 117 & 25.2 & 4.78 & 2.65 & 388 & 373 \\
\hline 08-04-05 & 1050 & 23.58 & 702 & 6.9 & 14.4 & -- & 383 & 113 & 24.4 & 4.51 & 2.43 & 384 & 385 \\
\hline \multicolumn{14}{|c|}{ MW2-1B (measuring point altitude 576.67 feet above NGVD 29) } \\
\hline 06-02-99 & 1400 & 12.42 & 885 & 7.0 & 14.9 & $<.1$ & 450 & 129 & 29.7 & 10.6 & 5.15 & -- & 466 \\
\hline 08-27-99 & 0900 & 18.69 & 830 & 6.8 & 14.6 & $<.1$ & 450 & 131 & 29.8 & 9.96 & 4.96 & -- & 456 \\
\hline 02-29-00 & 1600 & 23.19 & 732 & 6.9 & 15.1 & $<.1$ & 390 & 113 & 26.7 & 8.76 & 5.16 & 451 & 456 \\
\hline $08-29-00$ & 1400 & 22.09 & 827 & 7.0 & 15.7 & .1 & 500 & 140 & 35.9 & 9.82 & 4.85 & 463 & 490 \\
\hline 03-01-01 & 1010 & 21.00 & 871 & 7.0 & 13.6 & $<.1$ & 430 & 125 & 28.4 & 9.38 & 5.17 & 501 & 501 \\
\hline 08-23-01 & 0930 & 21.31 & 915 & 7.0 & 15.6 & $<.1$ & 460 & 133 & 31.4 & 10.9 & 5.31 & 392 & 406 \\
\hline $02-27-02$ & 0830 & 23.98 & 878 & 7.0 & 13.9 & $<.1$ & 430 & 125 & 29.1 & 10.3 & 5.34 & 494 & 493 \\
\hline $02-26-04$ & 0840 & 23.30 & 899 & 7.0 & 14.5 & $<.1$ & 490 & 142 & 32.1 & 11.2 & 5.18 & 370 & 357 \\
\hline $08-25-04$ & 1615 & 20.89 & 938 & 7.1 & 16.1 & -- & 510 & 150 & 32.7 & 12.2 & 5.74 & 516 & 299 \\
\hline 03-04-05 & 1315 & 20.87 & 926 & 7.0 & 15.0 & -- & 520 & 153 & 34.4 & 12.8 & 6.23 & 512 & 594 \\
\hline 08-04-05 & 1045 & 22.06 & 871 & 6.9 & 14.5 & -- & 467 & 137 & 30.4 & 11.4 & 5.46 & 524 & 528 \\
\hline
\end{tabular}


$\left[\mu \mathrm{S} / \mathrm{cm}\right.$, microsiemens per centimeter at 25 degrees Celsius; $\mathrm{pH}$, in standard units; ${ }^{\circ} \mathrm{C}$, degrees Celsius; constituent concentrations are dissolved, unless otherwise noted; mg/L, milligrams per liter; $\mathrm{CaCO}_{3}$, calcium carbonate; ANC, total acid neutralizing capacity, incremental titration; N, nitrogen; $\mu \mathrm{g} / \mathrm{L}$, micrograms per liter; NGVD 29, National Geodetic Vertical Datum of 1929; --, no data; $<$, less than; E, estimated; *, replicate sample; >, greater than]

\begin{tabular}{|c|c|c|c|c|c|c|c|c|c|c|c|c|c|}
\hline Date & Time & $\begin{array}{c}\text { Sulfate } \\
\text { (mg/L) }\end{array}$ & $\begin{array}{c}\text { Chloride } \\
\text { (mg/L) }\end{array}$ & $\begin{array}{l}\text { Fluoride } \\
\text { (mg/L) }\end{array}$ & $\begin{array}{l}\text { Residue, sum } \\
\text { of } \\
\text { constituents } \\
(\mathrm{mg} / \mathrm{L}))\end{array}$ & $\begin{array}{c}\text { Nitrite as N } \\
(\mathrm{mg} / \mathrm{L})\end{array}$ & $\begin{array}{c}\text { Nitrite plus } \\
\text { nitrate as N } \\
\text { (mg/L) }\end{array}$ & $\begin{array}{c}\text { Ammonia as } \\
\mathbf{N} \\
(\mathrm{mg} / \mathrm{L})\end{array}$ & $\begin{array}{c}\text { Ammonia } \\
\text { plus organic } \\
\mathrm{N} \text {, total } \\
\text { (mg/L) }\end{array}$ & $\begin{array}{c}\text { Phosphorous, } \\
\text { total } \\
\text { (mg/L) }\end{array}$ & $\begin{array}{l}\text { Phosphorous, } \\
\text { dissolved } \\
\text { (mg/L) }\end{array}$ & $\begin{array}{c}\text { Ortho- } \\
\text { phosphorous } \\
\text { (mg/L) }\end{array}$ & $\begin{array}{l}\text { Iron } \\
(\mu \mathrm{g} / \mathrm{L})\end{array}$ \\
\hline \multicolumn{14}{|c|}{ MW1-4B-Continued } \\
\hline $02-28-02$ & 0830 & 0.2 & 3.65 & 0.2 & 330 & $<0.008$ & $<0.05$ & 3.60 & 4.0 & 1.31 & 1.25 & 0.76 & 7,280 \\
\hline 08-20-02 & 1030 & .1 & 3.30 & .2 & 339 & $<.008$ & $<.05$ & 3.55 & 4.1 & 1.50 & 1.49 & 1.50 & 7,180 \\
\hline 02-26-04 & 1120 & $<.2$ & 3.39 & .2 & -- & E.004 & $<.06$ & .34 & 4.3 & 1.36 & 1.27 & 1.18 & 7,780 \\
\hline 08-24-04 & 1230 & .4 & 2.41 & .2 & 339 & $<.008$ & $<.06$ & 3.62 & 4.0 & 1.41 & 1.27 & E.01 & 7,780 \\
\hline 03-02-05 & 1415 & 31.1 & 5.14 & .3 & 410 & E.004 & E.06 & .91 & 1.1 & .38 & .09 & .05 & 548 \\
\hline 08-03-05 & 1410 & .9 & 2.62 & .2 & 345 & E.005 & $<.06$ & 3.13 & 3.6 & 1.35 & .82 & .83 & 5,390 \\
\hline \multicolumn{14}{|c|}{ MW2-1A } \\
\hline 06-02-99 & 1300 & 9.3 & 6.88 & .3 & 484 & $<.010$ & $<.05$ & .09 & .12 & E.03 & $<.05$ & .02 & 314 \\
\hline $08-27-99$ & 0810 & 15.3 & 6.79 & .3 & 460 & $<.010$ & .13 & $<.02$ & .10 & $<.05$ & $<.05$ & $<.01$ & 87 \\
\hline $02-29-00$ & 1520 & 14.3 & 6.33 & .3 & 411 & $<.010$ & .11 & $<.02$ & E.09 & $<.05$ & $<.05$ & $<.01$ & 223 \\
\hline $08-29-00$ & 1320 & 11.2 & 6.53 & .3 & 453 & $<.010$ & $<.05$ & .02 & E.06 & $<.05$ & $<.05$ & $<.01$ & 147 \\
\hline 03-01-01 & 0930 & 7.8 & 7.44 & .4 & 437 & $<.006$ & $<.05$ & .06 & .10 & $<.06$ & $<.06$ & $<.02$ & 281 \\
\hline 08-23-01 & 0830 & 9.8 & 6.80 & .3 & 400 & $<.006$ & E.04 & E.03 & .08 & $<.06$ & $<.06$ & E.02 & 150 \\
\hline $02-27-02$ & 0910 & 12.1 & 6.72 & .4 & 419 & $<.008$ & E.04 & E.03 & .13 & $<.06$ & $<.06$ & $<.02$ & 188 \\
\hline $08-22-02$ & 0900 & 9.6 & 5.37 & .3 & 391 & $<.008$ & .05 & E.03 & E.10 & $<.06$ & $<.06$ & $<.02$ & 503 \\
\hline 02-26-04 & 0920 & 14.5 & 5.70 & .4 & 365 & E.004 & E.03 & $<.04$ & E.09 & E.02 & $<.04$ & $<.18$ & 21 \\
\hline $08-25-04$ & 1535 & 13.7 & 3.98 & .3 & 414 & $<.008$ & E.04 & $<.04$ & E.09 & $<.04$ & E.02 & E.01 & 6 \\
\hline 03-04-05 & 1245 & 9.5 & 2.87 & .4 & 386 & $<.008$ & $<.06$ & $<.04$ & E.06 & $<.04$ & $<.04$ & $<.02$ & $<30$ \\
\hline 08-04-05 & 1050 & 9.2 & 2.27 & .3 & 387 & $<.008$ & E.04 & $<.04$ & .11 & $<.04$ & $<.04$ & $<.02$ & $<6$ \\
\hline \multicolumn{14}{|c|}{ MW2-1B } \\
\hline 06-02-99 & 1400 & 11.0 & 8.81 & .2 & 527 & .037 & $<.05$ & .59 & .55 & .64 & .47 & .44 & 13,000 \\
\hline $08-27-99$ & 0900 & 9.3 & 7.37 & .2 & 521 & .010 & $<.05$ & .47 & .64 & .69 & .47 & .30 & 13,500 \\
\hline $02-29-00$ & 1600 & 4.0 & 6.19 & .2 & 447 & $<.010$ & $<.05$ & .43 & .53 & .60 & .24 & .02 & 11,800 \\
\hline $08-29-00$ & 1400 & .6 & 5.35 & .2 & 492 & .017 & $<.05$ & .44 & .56 & .64 & $<.05$ & .33 & 15,000 \\
\hline 03-01-01 & 1010 & .4 & 6.50 & .3 & 489 & $<.006$ & $<.05$ & .45 & .56 & .55 & .56 & .02 & 12,800 \\
\hline 08-23-01 & 0930 & .4 & 5.94 & .2 & 436 & E.004 & $<.05$ & .48 & .64 & .65 & .52 & E.51 & 13,600 \\
\hline $02-27-02$ & 0830 & 1.1 & 6.78 & .3 & 487 & .026 & $<.05$ & .48 & .62 & .66 & .33 & $<.09$ & 12,800 \\
\hline 02-26-04 & 0840 & $<.2$ & 6.21 & .3 & -- & E.048 & $<.60$ & .41 & .60 & .64 & .59 & .64 & 13,600 \\
\hline $08-25-04$ & 1615 & $<.2$ & 5.38 & .3 & -- & $<.008$ & $<.06$ & .53 & .61 & .63 & .54 & $<.02$ & 4,700 \\
\hline 03-04-05 & 1315 & $<.2$ & 5.40 & .3 & -- & $<.008$ & $<.06$ & .48 & .62 & .64 & .22 & $<.02$ & 13,910 \\
\hline 08-04-05 & 1045 & $<.2$ & 5.54 & .3 & -- & $<.008$ & $<.06$ & .47 & .61 & .64 & .20 & $<.02$ & 11,750 \\
\hline
\end{tabular}


Table 1. Values of physical properties and concentrations of inorganic constituents and nutrients in samples from wells and surface-water sites, 1999-2005.-Continued

$\left[\mu \mathrm{S} / \mathrm{cm}\right.$, microsiemens per centimeter at 25 degrees Celsius; $\mathrm{pH}$, in standard units; ${ }^{\circ} \mathrm{C}$, degrees Celsius; constituent concentrations are dissolved, unless otherwise noted; mg/L, milligrams per liter; $\mathrm{CaCO}_{3}$, calcium carbonate; ANC, total acid neutralizing capacity, incremental titration; N, nitrogen; $\mu \mathrm{g} / \mathrm{L}$, micrograms per liter; NGVD 29, National Geodetic Vertical Datum of 1929; --, no data; $<$, less than; E, estimated; *, replicate sample; >, greater than]

\begin{tabular}{|c|c|c|c|c|c|c|c|c|c|c|c|c|c|}
\hline Date & Time & $\begin{array}{c}\text { Waterlevel } \\
\text { (feet below } \\
\text { measuring } \\
\text { point) }\end{array}$ & $\begin{array}{c}\text { Specific } \\
\text { conductance } \\
(\mu \mathrm{S} / \mathrm{cm})\end{array}$ & $\mathrm{pH}$ & $\begin{array}{c}\text { Temperature } \\
\left({ }^{\circ} \mathbf{C}\right)\end{array}$ & $\begin{array}{c}\text { Dissolved } \\
\text { oxygen } \\
\text { (mg/L) }\end{array}$ & $\begin{array}{c}\text { Hardness } \\
\text { (mg/L as } \\
\mathrm{CaCO}_{3} \text { ) }\end{array}$ & $\begin{array}{c}\text { Calcium } \\
\text { (mg/L) }\end{array}$ & $\begin{array}{l}\text { Magnesium } \\
(\mathbf{m g} / \mathbf{L})\end{array}$ & $\begin{array}{l}\text { Sodium } \\
\text { (mg/L) }\end{array}$ & $\begin{array}{l}\text { Potassium } \\
\text { (mg/L) }\end{array}$ & $\begin{array}{c}\text { Alkalinity, } \\
\text { fixed } \\
\text { endpoint } \\
\text { (mg/L) }\end{array}$ & $\begin{array}{c}\text { ANC } \\
\text { (mg/L) }\end{array}$ \\
\hline \multicolumn{14}{|c|}{ MW3-1A (measuring point altitude 572.53 feet above NGVD 29) } \\
\hline 06-02-99 & 1440 & 7.34 & 873 & 7.0 & 15.6 & $<0.1$ & 440 & 137 & 23.6 & 6.56 & 5.03 & -- & 439 \\
\hline 09-01-99 & 0900 & -- & 835 & 7.0 & 15.0 & $<.1$ & 460 & 145 & 24.7 & 6.63 & 4.54 & -- & 437 \\
\hline 03-08-00 & 0930 & 17.32 & 778 & 6.7 & 16.3 & $<.1$ & 410 & 128 & 21.7 & 6.41 & 4.45 & 386 & 386 \\
\hline $08-24-00$ & 1040 & 16.33 & 747 & 7.0 & 16.2 & $<.1$ & 390 & 122 & 20.6 & 7.41 & 4.51 & 349 & 357 \\
\hline $02-22-01$ & 1600 & 17.84 & -- & 7.1 & 14.8 & $<.1$ & 410 & 129 & 21.7 & 7.92 & 4.91 & 380 & 382 \\
\hline 08-23-01 & 1100 & 15.81 & 791 & 7.2 & 15.5 & $<.1$ & 410 & 129 & 21.7 & 8.96 & 4.55 & 313 & 310 \\
\hline $02-27-02$ & 1040 & 18.65 & 743 & 7.1 & 14.6 & $<.1$ & 390 & 122 & 19.8 & 8.31 & 4.27 & 373 & 378 \\
\hline 08-22-02 & 1340 & 17.74 & 774 & 7.1 & 17.1 & $<.1$ & 390 & 124 & 20.1 & 8.63 & 4.04 & 349 & 291 \\
\hline 04-01-03 & 1650 & 19.74 & 831 & 7.0 & 15.2 & $<.1$ & -- & -- & -- & -- & -- & -- & -- \\
\hline $08-27-03$ & 1430 & 19.61 & 1,000 & 6.9 & 15.9 & $<.1$ & -- & -- & -- & -- & -- & -- & -- \\
\hline $02-25-04$ & 1540 & 19.52 & 1,010 & 7.0 & 13.8 & -- & 520 & 157 & 31.0 & 8.85 & 6.14 & 246 & 250 \\
\hline 08-26-04 & 1340 & 16.35 & 844 & 6.8 & 17.0 & -- & 480 & 152 & 23.4 & 9.34 & 4.62 & 375 & 285 \\
\hline 03-04-05 & 1405 & 16.38 & 914 & 6.9 & 15.1 & -- & 510 & 164 & 25.1 & 9.49 & 5.04 & 464 & 530 \\
\hline 08-04-05 & 1210 & 18.02 & 957 & 6.9 & 14.4 & -- & 530 & 169 & 26.3 & 8.99 & 4.74 & 472 & 476 \\
\hline \multicolumn{14}{|c|}{ MW3-1B (measuring point altitude 572.51 feet above NGVD 29) } \\
\hline 06-02-99 & 1530 & 7.31 & 847 & 7.1 & 15.4 & $<.1$ & 440 & 129 & 28.3 & 7.62 & 4.18 & -- & 435 \\
\hline 09-01-99 & 0810 & -- & 807 & 7.0 & 14.7 & $<.1$ & 430 & 126 & 27.3 & 7.36 & 4.27 & -- & 432 \\
\hline 03-08-00 & 1030 & 17.32 & 775 & 6.8 & 15.7 & $<.1$ & 390 & 115 & 25.6 & 6.93 & 4.19 & 420 & 422 \\
\hline $08-24-00$ & 1130 & 16.31 & 802 & 7.0 & 16.0 & $<.1$ & 410 & 120 & 26.0 & 7.06 & 4.64 & 295 & 429 \\
\hline $02-22-01$ & 1640 & 17.88 & -- & 7.1 & 14.0 & $<.1$ & 460 & 137 & 29.5 & 7.41 & 4.42 & 486 & 485 \\
\hline $08-23-01$ & 1140 & 15.85 & 915 & 7.2 & 15.9 & $<.1$ & 470 & 140 & 29.8 & 7.93 & 4.74 & 373 & 357 \\
\hline $02-27-02$ & 1000 & 18.68 & 903 & 7.1 & 13.7 & $<.1$ & 460 & 137 & 28.1 & 7.60 & 4.57 & 489 & 492 \\
\hline 08-22-02 & 1430 & 17.78 & 967 & 7.1 & 17.0 & $<.1$ & 490 & 147 & 29.3 & 8.23 & 4.35 & 435 & 343 \\
\hline $02-25-04$ & 1620 & 19.55 & 826 & 7.0 & 14.4 & $<.1$ & 460 & 147 & 23.4 & 8.63 & 4.71 & 269 & 138 \\
\hline 08-26-04 & 1415 & 16.37 & 1,020 & 6.9 & 16.1 & -- & 550 & 168 & 31.1 & 9.51 & 5.10 & 472 & 407 \\
\hline * & 1420 & 16.37 & -- & 6.9 & -- & -- & -- & 161 & 31.5 & 9.56 & 5.09 & 472 & 380 \\
\hline 03-04-05 & 1435 & 16.44 & 1,120 & 7.0 & 14.7 & -- & 570 & 173 & 33.7 & 10.9 & 5.23 & 389 & 474 \\
\hline 08-04-05 & 1215 & 18.06 & 1,060 & 6.9 & 14.3 & -- & 553 & 168 & 32.5 & 11.0 & 5.23 & 405 & 408 \\
\hline
\end{tabular}


$\left[\mu \mathrm{S} / \mathrm{cm}\right.$, microsiemens per centimeter at 25 degrees Celsius; $\mathrm{pH}$, in standard units; ${ }^{\circ} \mathrm{C}$, degrees Celsius; constituent concentrations are dissolved, unless otherwise noted; mg/L, milligrams per liter; $\mathrm{CaCO}_{3}$, calcium carbonate; ANC, total acid neutralizing capacity, incremental titration; N, nitrogen; $\mu \mathrm{g} / \mathrm{L}$, micrograms per liter; NGVD 29, National Geodetic Vertical Datum of 1929; --, no data; $<$, less than; E, estimated; *, replicate sample; >, greater than]

\begin{tabular}{|c|c|c|c|c|c|c|c|c|c|c|c|c|c|}
\hline Date & Time & $\begin{array}{l}\text { Sulfate } \\
\text { (mg/L) }\end{array}$ & $\begin{array}{c}\text { Chloride } \\
\text { (mg/L) }\end{array}$ & $\begin{array}{c}\text { Fluoride } \\
\text { (mg/L) }\end{array}$ & $\begin{array}{l}\text { Residue, sum } \\
\text { of } \\
\text { constituents } \\
(\mathbf{m g} / \mathrm{L}))\end{array}$ & $\begin{array}{l}\text { Nitrite as N } \\
(\mathrm{mg} / \mathrm{L})\end{array}$ & $\begin{array}{c}\text { Nitrite plus } \\
\text { nitrate as N } \\
(\mathrm{mg} / \mathrm{L})\end{array}$ & $\begin{array}{c}\underset{\mathbf{N}}{\text { Ammonia as }} \\
(\mathbf{m g} / \mathrm{L})\end{array}$ & $\begin{array}{c}\text { Ammonia } \\
\text { plus organic } \\
\text { N, total } \\
(\mathrm{mg} / \mathrm{L})\end{array}$ & $\begin{array}{c}\text { Phosphorous, } \\
\text { total } \\
\text { (mg/L) }\end{array}$ & $\begin{array}{c}\text { Phosphorous, } \\
\text { dissolved } \\
\text { (mg/L) }\end{array}$ & $\begin{array}{c}\text { Ortho- } \\
\text { phosphorous } \\
\text { (mg/L) }\end{array}$ & $\begin{array}{l}\text { Iron } \\
(\mu \mathrm{g} / \mathrm{L})\end{array}$ \\
\hline \multicolumn{14}{|c|}{ MW3-1A } \\
\hline 06-02-99 & 1440 & 40.8 & 11.8 & 0.3 & 512 & $<0.010$ & $<0.05$ & 0.06 & 0.13 & E0.04 & $<0.05$ & 0.03 & $<10$ \\
\hline 09-01-99 & 0900 & 43.1 & 9.30 & .3 & 522 & $<.010$ & $<.05$ & $<.02$ & E.07 & $<.05$ & $<.05$ & .02 & $<10$ \\
\hline $03-08-00$ & 0930 & 60.1 & 11.6 & .3 & 464 & $<.010$ & $<.05$ & .03 & .14 & $<.05$ & E.03 & .02 & $<10$ \\
\hline $08-24-00$ & 1040 & 59.4 & 12.2 & .3 & 435 & $<.010$ & $<.05$ & $<.02$ & .10 & $<.05$ & $<.05$ & .02 & $<10$ \\
\hline $02-22-01$ & 1600 & 61.0 & 9.27 & .3 & 462 & $<.006$ & $<.05$ & $<.04$ & .09 & E.03 & E.04 & .02 & E6 \\
\hline 08-23-01 & 1100 & 54.0 & 8.30 & .3 & 415 & $<.006$ & $<.05$ & E.02 & .12 & $<.06$ & $<.06$ & E.02 & E8 \\
\hline $02-27-02$ & 1040 & 46.4 & 7.44 & .3 & 432 & $<.008$ & $<.05$ & E.02 & .11 & E. 05 & $<.06$ & $<.02$ & $<10$ \\
\hline $08-22-02$ & 1340 & 44.9 & 7.91 & .3 & 420 & $<.008$ & $<.05$ & $<.04$ & E.09 & $<.06$ & $<.06$ & .02 & 12 \\
\hline 04-01-03 & 1650 & -- & 7.91 & -- & -- & -- & -- & -- & -- & -- & -- & -- & -- \\
\hline $08-27-03$ & 1430 & -- & 25.6 & -- & -- & -- & -- & -- & -- & -- & -- & -- & -- \\
\hline $02-25-04$ & 1540 & 28.8 & 46.2 & .2 & 436 & -- & -- & -- & -- & -- & -- & -- & -- \\
\hline 08-26-04 & 1340 & 35.6 & 7.01 & .3 & 457 & $<.008$ & $<.06$ & E.02 & .14 & .05 & E. 04 & .02 & 10 \\
\hline 03-04-05 & 1405 & 52.0 & 7.79 & .3 & 582 & $<.008$ & $<.06$ & $<.04$ & E.09 & .04 & E.03 & .02 & 41 \\
\hline 08-04-05 & 1210 & 55.4 & 11.2 & .3 & 562 & $<.008$ & $<.06$ & $<.04$ & E.09 & E.03 & E.03 & $<.02$ & 7 \\
\hline \multicolumn{14}{|c|}{ MW3-1B } \\
\hline 06-02-99 & 1530 & 23.1 & 5.64 & .2 & 511 & .040 & $<.05$ & .55 & .47 & .48 & .50 & .38 & 12,900 \\
\hline 09-01-99 & 0810 & 20.8 & 5.93 & .2 & 503 & .010 & $<.05$ & .38 & .41 & .47 & .38 & .56 & 13,000 \\
\hline 03-08-00 & 1030 & 26.2 & 5.08 & .2 & 449 & .010 & $<.05$ & .37 & .50 & .47 & E.03 & .33 & 11,700 \\
\hline $08-24-00$ & 1130 & 21.6 & 4.84 & .2 & 375 & .020 & $<.05$ & .35 & .42 & .48 & .48 & .43 & 12,100 \\
\hline $02-22-01$ & 1640 & 21.6 & 5.25 & .3 & 511 & $<.006$ & $<.05$ & .35 & .48 & .48 & .57 & .04 & 13,900 \\
\hline 08-23-01 & 1140 & 22.0 & 5.70 & .2 & 448 & E.023 & E.03 & E. 40 & .49 & .48 & .39 & E. 38 & 14,000 \\
\hline $02-27-02$ & 1000 & 20.7 & 5.01 & .3 & 512 & .020 & $<.05$ & .41 & .48 & .49 & .42 & .43 & 14,000 \\
\hline $08-22-02$ & 1430 & 21.0 & 6.73 & .2 & 495 & .020 & $<.05$ & .41 & .48 & .51 & .52 & .53 & 14,500 \\
\hline $02-25-04$ & 1620 & 37.9 & 7.73 & .3 & 391 & -- & -- & -- & -- & -- & -- & -- & -- \\
\hline 08-26-04 & 1415 & 29.4 & 33.9 & .2 & 577 & $<.008$ & $<.06$ & .41 & .52 & .51 & .14 & $<.02$ & 15,800 \\
\hline$*$ & 1420 & 29.3 & 33.9 & .2 & -- & $<.008$ & $<.06$ & .40 & .53 & .49 & .21 & $<.02$ & 15,700 \\
\hline 03-04-05 & 1435 & 52.6 & 99.4 & .3 & 677 & $<.008$ & $<.06$ & .38 & .54 & .37 & .22 & $<.02$ & 17,190 \\
\hline 08-04-05 & 1215 & 54.7 & 98.7 & .2 & 631 & $<.008$ & $<.06$ & .37 & .53 & .44 & E.02 & $<.02$ & 15,470 \\
\hline
\end{tabular}


Table 1. Values of physical properties and concentrations of inorganic constituents and nutrients in samples from wells and surface-water sites, 1999-2005.-Continued

$\left[\mu \mathrm{S} / \mathrm{cm}\right.$, microsiemens per centimeter at 25 degrees Celsius; $\mathrm{pH}$, in standard units; ${ }^{\circ} \mathrm{C}$, degrees Celsius; constituent concentrations are dissolved, unless otherwise noted; mg/L, milligrams per liter; $\mathrm{CaCO}_{3}$, calcium carbonate; ANC, total acid neutralizing capacity, incremental titration; N, nitrogen; $\mu \mathrm{g} / \mathrm{L}$, micrograms per liter; NGVD 29, National Geodetic Vertical Datum of 1929; --, no data; $<$, less than; E, estimated; *, replicate sample; >, greater than]

\begin{tabular}{|c|c|c|c|c|c|c|c|c|c|c|c|c|c|}
\hline Date & Time & $\begin{array}{c}\text { Waterlevel } \\
\text { (feet below } \\
\text { measuring } \\
\text { point) }\end{array}$ & $\begin{array}{c}\text { Specific } \\
\text { conductance } \\
(\mu \mathrm{S} / \mathrm{cm})\end{array}$ & $\mathrm{pH}$ & $\begin{array}{c}\text { Temperature } \\
\left({ }^{\circ} \mathbf{C}\right)\end{array}$ & $\begin{array}{c}\text { Dissolved } \\
\text { oxygen } \\
\text { (mg/L) }\end{array}$ & $\begin{array}{c}\text { Hardness } \\
\text { (mg/L as } \\
\left.\mathrm{CaCO}_{3}\right)\end{array}$ & $\begin{array}{c}\text { Calcium } \\
\text { (mg/L) }\end{array}$ & $\begin{array}{l}\text { Magnesium } \\
(\mathbf{m g} / \mathbf{L})\end{array}$ & $\begin{array}{l}\text { Sodium } \\
\text { (mg/L) }\end{array}$ & $\begin{array}{l}\text { Potassium } \\
\text { (mg/L) }\end{array}$ & $\begin{array}{l}\text { Alkalinity, } \\
\text { fixed } \\
\text { endpoint } \\
\text { (mg/L) }\end{array}$ & $\begin{array}{c}\text { ANC } \\
\text { (mg/L) }\end{array}$ \\
\hline \multicolumn{14}{|c|}{ MW4-1A (measuring point altitude 572.66 feet above NGVD 29) } \\
\hline 06-02-99 & 0810 & 6.07 & 958 & 6.9 & 14.0 & $<0.1$ & 470 & 127 & 36.7 & 9.18 & 5.55 & -- & 413 \\
\hline 08-31-99 & 1300 & -- & 889 & 7.1 & 14.1 & $<.1$ & 490 & 137 & 36.4 & 7.50 & 5.17 & -- & 437 \\
\hline 03-01-00 & 1530 & 15.49 & 899 & 6.9 & 13.8 & $<.1$ & 450 & 121 & 35.0 & 14.6 & 5.80 & 434 & 439 \\
\hline $08-25-00$ & 1030 & 15.29 & 901 & 7.0 & 15.1 & $<.1$ & 410 & 114 & 31.3 & 17.8 & 5.60 & 362 & 369 \\
\hline 02-23-01 & 0800 & 16.53 & -- & 7.0 & 13.0 & $<.1$ & 460 & 129 & 34.2 & 14.9 & 5.46 & 453 & 454 \\
\hline $08-22-01$ & 0840 & 14.36 & 965 & 6.9 & 15.1 & $<.1$ & 500 & 138 & 36.9 & 15.9 & 5.41 & 412 & 359 \\
\hline $02-26-02$ & 1420 & 17.01 & 959 & 7.0 & 13.2 & $<.1$ & 470 & 133 & 32.6 & 15.9 & 5.64 & 474 & 478 \\
\hline $08-21-02$ & 1130 & 17.20 & 996 & 7.1 & 16.0 & $<.1$ & 530 & 151 & 36.2 & 15.5 & 5.32 & 496 & 448 \\
\hline $04-02-03$ & 0950 & 18.41 & 969 & 7.1 & 14.7 & $<.1$ & -- & -- & -- & -- & -- & -- & -- \\
\hline $08-27-03$ & 1230 & 18.66 & 1.030 & 7.1 & 15.6 & $<.1$ & -- & -- & -- & -- & -- & -- & -- \\
\hline $02-25-04$ & 1440 & 18.47 & 1,060 & 7.0 & 13.5 & $<.1$ & 510 & 147 & 35.0 & 20.2 & 6.16 & 396 & 250 \\
\hline 08-26-04 & 1210 & 15.09 & 1,080 & 6.9 & 16.3 & -- & 550 & 163 & 35.4 & 19.3 & 6.17 & 478 & 466 \\
\hline 03-04-05 & 0945 & 14.48 & 1,070 & 7.0 & 14.3 & -- & 550 & 164 & 34.9 & 19.4 & 6.13 & 492 & 515 \\
\hline 08-04-05 & 1340 & 16.99 & 1,020 & 7.4 & 20.1 & -- & 534 & 158 & 34.0 & 14.2 & 5.61 & 538 & 546 \\
\hline \multicolumn{14}{|c|}{ MW4-1B (measuring point altitude 572.48 feet above NGVD 29) } \\
\hline 06-02-99 & 0915 & 5.91 & 798 & 7.1 & 14.4 & 0.4 & 390 & 106 & 29.1 & 10.8 & 3.94 & -- & 269 \\
\hline 08-31-99 & 1350 & -- & 759 & 7.1 & 14.3 & $<.1$ & 380 & 107 & 27.6 & 14.6 & 4.05 & -- & 255 \\
\hline $03-01-00$ & 1610 & 15.36 & 776 & 7.0 & 13.2 & $<.1$ & 350 & 96.6 & 27.1 & 15.9 & 4.39 & 307 & 311 \\
\hline $08-25-00$ & 1120 & 15.13 & 873 & 7.0 & 14.9 & $<.1$ & 400 & 110 & 30.4 & 12.2 & 4.67 & -- & 377 \\
\hline $02-23-01$ & 0810 & 16.47 & -- & 7.1 & 12.8 & $<.1$ & 420 & 116 & 30.9 & 13.7 & 4.28 & 376 & 373 \\
\hline $08-22-01$ & 0920 & 14.29 & 876 & 7.0 & 14.9 & $<.1$ & 420 & 115 & 31.4 & 14.2 & 4.50 & 365 & 366 \\
\hline $02-26-02$ & 1510 & 16.86 & 993 & 7.1 & 13.0 & $<.1$ & 450 & 127 & 32.9 & 15.5 & 4.90 & 372 & 373 \\
\hline 08-21-02 & 1230 & 16.99 & 1,110 & 7.1 & 16.0 & $<.1$ & 510 & 144 & 36.6 & 24.3 & 5.01 & 356 & 286 \\
\hline $02-25-04$ & 1400 & 18.33 & 1,270 & 7.0 & 13.4 & $<.1$ & 500 & 141 & 34.8 & 66.5 & 5.82 & 247 & 214 \\
\hline 08-26-04 & 1255 & 14.86 & 1,380 & 7.0 & 16.0 & -- & 460 & 132 & 31.8 & 90.6 & 6.04 & 332 & 282 \\
\hline 03-04-05 & 1020 & 14.49 & 1,320 & 7.1 & 14.3 & -- & 480 & 137 & 34.3 & 90.3 & 6.32 & 360 & 394 \\
\hline 08-04-05 & 1330 & 16.95 & 1,250 & 7.1 & 14.2 & -- & 423 & 122 & 28.6 & 95.0 & 5.57 & 386 & 386 \\
\hline
\end{tabular}


$\left[\mu \mathrm{S} / \mathrm{cm}\right.$, microsiemens per centimeter at 25 degrees Celsius; $\mathrm{pH}$, in standard units; ${ }^{\circ} \mathrm{C}$, degrees Celsius; constituent concentrations are dissolved, unless otherwise noted; mg/L, milligrams per liter; $\mathrm{CaCO}_{3}$, calcium carbonate; ANC, total acid neutralizing capacity, incremental titration; N, nitrogen; $\mu \mathrm{g} / \mathrm{L}$, micrograms per liter; NGVD 29, National Geodetic Vertical Datum of 1929; --, no data; $<$, less than; E, estimated; *, replicate sample; >, greater than]

\begin{tabular}{|c|c|c|c|c|c|c|c|c|c|c|c|c|c|}
\hline Date & Time & $\begin{array}{l}\text { Sulfate } \\
\text { (mg/L) }\end{array}$ & $\begin{array}{c}\text { Chloride } \\
\text { (mg/L) }\end{array}$ & $\begin{array}{c}\text { Fluoride } \\
\text { (mg/L) }\end{array}$ & $\begin{array}{l}\text { Residue, sum } \\
\text { of } \\
\text { constituents } \\
(\mathrm{mg} / \mathrm{L}))\end{array}$ & $\begin{array}{l}\text { Nitrite as N } \\
(\mathbf{m g} / \mathbf{L})\end{array}$ & $\begin{array}{c}\text { Nitrite plus } \\
\text { nitrate as N } \\
\text { (mg/L) }\end{array}$ & $\begin{array}{c}\text { Ammonia as } \\
\mathbf{N} \\
(\mathrm{mg} / \mathrm{L})\end{array}$ & $\begin{array}{c}\text { Ammonia } \\
\text { plus organic } \\
\mathrm{N}, \text { total } \\
\text { (mg/L) }\end{array}$ & $\begin{array}{c}\text { Phosphorous, } \\
\text { total } \\
\text { (mg/L) }\end{array}$ & $\begin{array}{l}\text { Phosphorous, } \\
\text { dissolved } \\
\text { (mg/L) }\end{array}$ & $\begin{array}{c}\text { Ortho- } \\
\text { phosphorous } \\
\text { (mg/L) }\end{array}$ & $\begin{array}{l}\text { Iron } \\
(\mu \mathrm{g} / \mathrm{L})\end{array}$ \\
\hline \multicolumn{14}{|c|}{ MW4-1A } \\
\hline 06-02-99 & 0810 & 88.8 & 14.1 & 0.2 & 577 & 0.022 & $<0.05$ & 0.97 & 0.96 & 0.47 & 0.08 & 0.34 & 13,200 \\
\hline 08-31-99 & 1300 & 60.9 & 10.7 & .2 & 568 & .020 & .23 & .74 & .84 & .30 & .29 & .30 & 11,400 \\
\hline $03-01-00$ & 1530 & 74.0 & 12.6 & .2 & 539 & .015 & $<.05$ & .81 & .99 & .52 & $<.05$ & .47 & 12,800 \\
\hline $08-25-00$ & 1030 & 94.7 & 18.7 & .2 & 514 & .011 & $<.05$ & .80 & .92 & .49 & .25 & .38 & 12,300 \\
\hline 02-23-01 & 0800 & 61.9 & 9.64 & .2 & 543 & E.004 & $<.05$ & .87 & 1.0 & .52 & .51 & .08 & 14,900 \\
\hline 08-22-01 & 0840 & 61.9 & 11.6 & .2 & 533 & .008 & E.02 & .89 & .95 & .45 & .53 & E.44 & 15,300 \\
\hline $02-26-02$ & 1420 & 60.1 & 11.8 & .2 & 563 & .023 & $<.05$ & .93 & 1.0 & .50 & .56 & .48 & 16,200 \\
\hline $08-21-02$ & 1130 & 58.0 & 11.3 & .2 & 594 & .017 & $<.05$ & .91 & 1.1 & .52 & .56 & .50 & 16,500 \\
\hline $04-02-03$ & 0950 & -- & 12.3 & -- & -- & -- & -- & -- & -- & -- & -- & -- & -- \\
\hline $08-27-03$ & 1230 & -- & 17.0 & -- & -- & -- & -- & -- & -- & -- & -- & -- & -- \\
\hline $02-25-04$ & 1440 & 80.8 & 23.7 & .2 & 571 & E.052 & $<.60$ & .77 & 1.1 & .62 & .58 & .45 & 17,800 \\
\hline 08-26-04 & 1210 & 77.9 & 19.3 & .2 & 627 & $<.008$ & $<.06$ & .88 & 1.0 & .59 & .09 & $<.02$ & 18,000 \\
\hline 03-04-05 & 0945 & 67.7 & 20.8 & .2 & 643 & $<.008$ & $<.06$ & .78 & .96 & .60 & .36 & $<.02$ & 19,610 \\
\hline 08-04-05 & 1340 & 58.5 & 14.5 & .2 & 630 & $<.008$ & $<.06$ & .78 & .98 & .52 & .34 & $<.02$ & 16,450 \\
\hline \multicolumn{14}{|c|}{ MW4-1B } \\
\hline 06-02-99 & 0915 & 122 & 18.4 & .2 & 503 & 0.034 & $<.05$ & .98 & .95 & .80 & $<.05$ & .61 & 13,000 \\
\hline 08-31-99 & 1350 & 121 & 19.8 & .2 & 508 & .057 & $<.05$ & .84 & .83 & .79 & .80 & .96 & 18,000 \\
\hline $03-01-00$ & 1610 & 97.8 & 15.4 & .2 & 461 & .027 & $<.05$ & .85 & 1.0 & .83 & $<.05$ & .58 & 16,500 \\
\hline $08-25-00$ & 1120 & 69.4 & 21.0 & .2 & 494 & .019 & $<.05$ & .89 & .99 & .83 & .33 & .51 & 17,800 \\
\hline 02-23-01 & 0810 & 76.5 & 17.9 & .2 & 505 & E.003 & $<.05$ & .87 & .99 & .84 & 1.03 & .08 & 18,700 \\
\hline 08-22-01 & 0920 & 83.1 & 24.6 & .2 & 513 & E.041 & E.04 & E.95 & 1.0 & .82 & E. 88 & E.68 & 20,200 \\
\hline $02-26-02$ & 1510 & 72.5 & 63.3 & .2 & 563 & .044 & $<.05$ & 1.07 & 1.1 & .81 & .76 & .49 & 20,300 \\
\hline $08-21-02$ & 1230 & 71.2 & 105 & .2 & 624 & .066 & $<.05$ & 1.11 & 1.2 & .79 & .84 & .85 & 20,600 \\
\hline $02-25-04$ & 1400 & 61.1 & 163 & .2 & 644 & .083 & $<.60$ & .88 & 1.2 & .81 & .71 & .68 & 19,700 \\
\hline $08-26-04$ & 1255 & 68.3 & 188 & .2 & 735 & $<.008$ & $<.06$ & 1.11 & 1.2 & .74 & .25 & $<.02$ & 17,700 \\
\hline 03-04-05 & 1020 & 66.1 & 166 & .2 & 760 & $<.008$ & $<.06$ & 1.06 & 1.3 & .84 & .47 & $<.02$ & 21,970 \\
\hline 08-04-05 & 1330 & 77.9 & 163 & .2 & 743 & $<.008$ & $<.06$ & 1.03 & 1.2 & .89 & .25 & $<.02$ & 17,620 \\
\hline
\end{tabular}


Table 1. Values of physical properties and concentrations of inorganic constituents and nutrients in samples from wells and surface-water sites, 1999-2005.-Continued

$\left[\mu \mathrm{S} / \mathrm{cm}\right.$, microsiemens per centimeter at 25 degrees Celsius; $\mathrm{pH}$, in standard units; ${ }^{\circ} \mathrm{C}$, degrees Celsius; constituent concentrations are dissolved, unless otherwise noted; mg/L, milligrams per liter; $\mathrm{CaCO}_{3}$, calcium carbonate; ANC, total acid neutralizing capacity, incremental titration; N, nitrogen; $\mu \mathrm{g} / \mathrm{L}$, micrograms per liter; NGVD 29, National Geodetic Vertical Datum of 1929; --, no data; $<$, less than; E, estimated; *, replicate sample; >, greater than]

\begin{tabular}{|c|c|c|c|c|c|c|c|c|c|c|c|c|c|}
\hline Date & Time & $\begin{array}{c}\text { Waterlevel } \\
\text { (feet below } \\
\text { measuring } \\
\text { point) }\end{array}$ & $\begin{array}{c}\text { Specific } \\
\text { conductance } \\
(\mu \mathrm{S} / \mathrm{cm})\end{array}$ & $\mathrm{pH}$ & $\begin{array}{c}\text { Temperature } \\
\left({ }^{\circ} \mathrm{C}\right)\end{array}$ & $\begin{array}{c}\text { Dissolved } \\
\text { oxygen } \\
\text { (mg/L) }\end{array}$ & $\begin{array}{c}\text { Hardness } \\
\text { (mg/L as } \\
\mathrm{CaCO}_{3} \text { ) }\end{array}$ & $\begin{array}{l}\text { Calcium } \\
\text { (mg/L) }\end{array}$ & $\begin{array}{l}\text { Magnesium } \\
(\mathbf{m g} / \mathbf{L})\end{array}$ & $\begin{array}{l}\text { Sodium } \\
\text { (mg/L) }\end{array}$ & $\begin{array}{l}\text { Potassium } \\
\text { (mg/L) }\end{array}$ & $\begin{array}{c}\text { Alkalinity, } \\
\text { fixed } \\
\text { endpoint } \\
\text { (mg/L) }\end{array}$ & $\begin{array}{l}\text { ANC } \\
\text { (mg/L) }\end{array}$ \\
\hline \multicolumn{14}{|c|}{ MW4-2A (measuring point altitude 572.12 feet above NGVD 29) } \\
\hline 06-02-99 & 1100 & 5.40 & 963 & 7.0 & 14.8 & $<0.1$ & 460 & 137 & 29.0 & 10.5 & 6.52 & -- & 465 \\
\hline 08-31-99 & 1500 & -- & 968 & 7.0 & 14.4 & $<.1$ & 520 & 156 & 31.3 & 9.94 & 5.80 & -- & 491 \\
\hline 03-01-00 & 1340 & 12.80 & 1,060 & 6.9 & 14.5 & $<.1$ & 480 & 142 & 30.4 & 31.1 & 7.36 & 461 & 467 \\
\hline $08-25-00$ & 0840 & 13.21 & 1,200 & 7.0 & 16.3 & .4 & 470 & 138 & 30.1 & 51.4 & 7.97 & 404 & 412 \\
\hline 02-23-01 & 0950 & 14.32 & -- & 7.1 & 14.1 & $<.1$ & 440 & 130 & 27.3 & 36.6 & 6.76 & 437 & 436 \\
\hline 08-22-01 & 1020 & 12.72 & 1,140 & 7.0 & 16.0 & $<.1$ & 450 & 132 & 28.7 & 61.7 & 6.73 & 433 & 354 \\
\hline $02-26-02$ & 1300 & 14.89 & 1,180 & 7.0 & 15.3 & $<.1$ & 400 & 119 & 24.6 & 87.4 & 7.86 & 379 & 388 \\
\hline 08-21-02 & 1400 & 15.14 & 1,240 & 7.1 & 16.8 & $<.1$ & 440 & 132 & 26.3 & 96.5 & 7.15 & 408 & 358 \\
\hline $04-02-03$ & 0850 & 16.55 & 1,140 & 7.1 & 16.7 & $<.1$ & -- & -- & -- & -- & -- & -- & -- \\
\hline $08-27-03$ & 1130 & 17.17 & 1,120 & 7.0 & 17.2 & $<.1$ & -- & -- & -- & -- & -- & -- & -- \\
\hline $02-25-04$ & 1110 & 16.68 & 1,190 & 7.0 & 15.7 & $<.1$ & 380 & 115 & 23.7 & 101 & 7.50 & 310 & 276 \\
\hline 08-26-04 & 1045 & 13.03 & 1,260 & 7.2 & 17.9 & .6 & 460 & 138 & 27.2 & 91.7 & 8.07 & 355 & 408 \\
\hline 03-04-05 & 1115 & 12.40 & 1,220 & 6.9 & 16.9 & .2 & 450 & 135 & 26.9 & 90.7 & 8.60 & 389 & 514 \\
\hline 08-04-05 & 1445 & 15.62 & 1,120 & 7.7 & 22.7 & .5 & 450 & 136 & 27.9 & 71.6 & 6.83 & 430 & 434 \\
\hline \multicolumn{14}{|c|}{ MW4-2B (measuring point altitude 572.14 feet above NGVD 29) } \\
\hline 06-02-99 & 1020 & 5.39 & 1,060 & 6.9 & 14.7 & $<.1$ & 490 & 145 & 31.4 & 13.1 & 5.61 & -- & 409 \\
\hline 08-31-99 & 1600 & -- & 1,050 & 6.9 & 14.6 & $<.1$ & 500 & 147 & 31.9 & 15.9 & 5.79 & -- & 376 \\
\hline 03-01-00 & 1430 & 12.82 & 1,010 & 6.9 & 14.1 & $<.1$ & 440 & 130 & 28.9 & 24.3 & 5.96 & 350 & 354 \\
\hline $08-25-00$ & 0930 & 13.32 & 1,110 & 7.0 & 16.0 & $<.1$ & 400 & 117 & 26.5 & 49.8 & 6.65 & 317 & 324 \\
\hline 02-23-01 & 1030 & 14.36 & -- & 7.1 & 14.2 & $<.1$ & 410 & 121 & 26.3 & 88.4 & 6.52 & 252 & 253 \\
\hline $08-22-01$ & 1100 & 12.72 & 1,320 & 7.1 & 16.4 & $<.1$ & 350 & 101 & 23.2 & 123 & 6.37 & 289 & 287 \\
\hline $02-26-02$ & 1330 & 14.89 & 1,260 & 7.1 & 14.6 & $<.1$ & 340 & 101 & 21.6 & 117 & 6.79 & 283 & 282 \\
\hline 08-21-02 & 1510 & 15.15 & 1,200 & 7.1 & 18.7 & $<.1$ & 330 & 96.7 & 20.4 & 122 & 6.62 & 247 & 329 \\
\hline $02-25-04$ & 1200 & 16.67 & 1,360 & 7.0 & 15.3 & -- & 400 & 119 & 23.9 & 124 & 7.28 & 202 & 152 \\
\hline 08-26-04 & 1120 & 13.06 & 1,330 & 7.3 & 18.1 & -- & 340 & 102 & 19.7 & 141 & 7.35 & 297 & 291 \\
\hline 03-04-05 & 1155 & 12.43 & 1,390 & 7.1 & 16.1 & -- & 330 & 100 & 19.4 & 153 & 7.42 & 249 & 263 \\
\hline 08-04-05 & 1440 & 15.66 & 1,370 & 7.2 & 17.1 & -- & 296 & 91.2 & 16.5 & 162 & 7.54 & 319 & 320 \\
\hline * & 1445 & 15.66 & 1,370 & 7.2 & 17.1 & -- & 294 & 90.7 & 16.4 & 161 & 7.33 & 319 & 320 \\
\hline
\end{tabular}


$\left[\mu \mathrm{S} / \mathrm{cm}\right.$, microsiemens per centimeter at 25 degrees Celsius; $\mathrm{pH}$, in standard units; ${ }^{\circ} \mathrm{C}$, degrees Celsius; constituent concentrations are dissolved, unless otherwise noted; mg/L, milligrams per liter; $\mathrm{CaCO}_{3}$, calcium carbonate; ANC, total acid neutralizing capacity, incremental titration; N, nitrogen; $\mu \mathrm{g} / \mathrm{L}$, micrograms per liter; NGVD 29, National Geodetic Vertical Datum of 1929; --, no data; $<$, less than; E, estimated; *, replicate sample; >, greater than]

\begin{tabular}{|c|c|c|c|c|c|c|c|c|c|c|c|c|c|}
\hline Date & Time & $\begin{array}{l}\text { Sulfate } \\
\text { (mg/L) }\end{array}$ & $\begin{array}{c}\text { Chloride } \\
\text { (mg/L) }\end{array}$ & $\begin{array}{c}\text { Fluoride } \\
\text { (mg/L) }\end{array}$ & $\begin{array}{c}\text { Residue, sum } \\
\text { of } \\
\text { constituents } \\
(\mathrm{mg} / \mathrm{L}))\end{array}$ & $\begin{array}{l}\text { Nitrite as N } \\
\quad(\mathrm{mg} / \mathrm{L})\end{array}$ & $\begin{array}{c}\text { Nitrite plus } \\
\text { nitrate as N } \\
\quad(\mathrm{mg} / \mathrm{L})\end{array}$ & $\begin{array}{c}\text { Ammonia as } \\
\mathbf{N} \\
(\mathrm{mg} / \mathrm{L})\end{array}$ & $\begin{array}{l}\text { Ammonia } \\
\text { plus organic } \\
\text { N, total } \\
\text { (mg/L) }\end{array}$ & $\begin{array}{c}\text { Phosphorous, } \\
\text { total } \\
\text { (mg/L) }\end{array}$ & $\begin{array}{l}\text { Phosphorous, } \\
\text { dissolved } \\
\text { (mg/L) }\end{array}$ & $\begin{array}{c}\text { Ortho- } \\
\text { phosphorous } \\
\text { (mg/L) }\end{array}$ & $\begin{array}{l}\text { Iron } \\
(\mu \mathrm{g} / \mathrm{L})\end{array}$ \\
\hline \multicolumn{14}{|c|}{ MW4-2A } \\
\hline 06-02-99 & 1100 & 37.8 & 19.9 & 0.2 & 560 & 0.023 & $<0.05$ & 0.68 & 0.64 & 0.19 & $<0.05$ & 0.13 & 10,300 \\
\hline 08-31-99 & 1500 & 30.8 & 26.3 & .1 & 598 & .049 & .46 & .49 & .55 & .10 & .11 & .14 & 8,760 \\
\hline 03-01-00 & 1340 & 49.3 & 71.9 & $<.1$ & 623 & .019 & $<.05$ & .59 & .75 & .22 & $<.05$ & .05 & 13,400 \\
\hline $08-25-00$ & 0840 & 48.8 & 121 & .1 & 652 & .019 & $<.05$ & .86 & .98 & .19 & .16 & .18 & 10,600 \\
\hline 02-23-01 & 0950 & 37.1 & 61.1 & .2 & 573 & .007 & $<.05$ & .70 & .80 & .26 & .24 & .13 & 10,700 \\
\hline 08-22-01 & 1020 & 45.6 & 106 & .2 & 651 & .011 & $<.05$ & E.72 & .89 & .30 & .14 & .16 & 10,200 \\
\hline $02-26-02$ & 1300 & 59.6 & 143 & .2 & 683 & .027 & $<.05$ & 1.25 & 1.30 & .37 & .38 & .30 & 10,900 \\
\hline $08-21-02$ & 1400 & 62.8 & 142 & .3 & 724 & E.007 & $<.05$ & 1.30 & 1.40 & .40 & .47 & .12 & 10,200 \\
\hline $04-02-03$ & 0850 & -- & 118 & -- & -- & -- & -- & -- & -- & -- & -- & -- & -- \\
\hline $08-27-03$ & 1130 & -- & 111 & -- & -- & -- & -- & -- & -- & -- & -- & -- & -- \\
\hline $02-25-04$ & 1110 & 59.0 & 126 & .3 & 625 & E.004 & $<.06$ & .08 & 1.00 & .28 & .25 & E. 25 & 6,370 \\
\hline 08-26-04 & 1045 & 57.1 & 116 & .3 & 661 & $<.008$ & $<.06$ & 1.11 & 1.30 & .38 & .10 & $<.02$ & 8,440 \\
\hline 03-04-05 & 1115 & 45.2 & 139 & .3 & 766 & $<.008$ & $<.06$ & 1.07 & 1.28 & .42 & .27 & $<.02$ & 9,700 \\
\hline 08-04-05 & 1445 & 39.9 & 100 & .3 & 648 & $<.008$ & .30 & .65 & .95 & .18 & .09 & .04 & 4,540 \\
\hline \multicolumn{14}{|c|}{ MW4-2B } \\
\hline 06-02-99 & 1020 & 35.9 & 71.6 & .1 & 611 & .016 & $<.05$ & .66 & .64 & .71 & .16 & .14 & 19,600 \\
\hline 08-31-99 & 1600 & 37.8 & 98.4 & .1 & 631 & .039 & $<.05$ & .57 & .62 & .65 & .28 & .60 & 22,200 \\
\hline 03-01-00 & 1430 & 52.9 & 100 & .1 & 571 & .024 & $<.05$ & .57 & .71 & .64 & $<.05$ & .41 & 16,000 \\
\hline $08-25-00$ & 0930 & 71.9 & 107 & .2 & 590 & .019 & $<.05$ & .65 & .76 & .69 & .30 & .66 & 17,200 \\
\hline 02-23-01 & 1030 & 92.0 & 218 & .2 & 721 & .023 & $<.05$ & .70 & .86 & .67 & .64 & .41 & 15,500 \\
\hline $08-22-01$ & 1100 & 77.7 & 204 & .2 & 724 & E.037 & E.03 & E.78 & .93 & .71 & E.68 & E. 49 & 14,500 \\
\hline $02-26-02$ & 1330 & 94.5 & 183 & .2 & 709 & .039 & $<.05$ & .77 & .94 & .72 & .58 & .56 & 12,900 \\
\hline $08-21-02$ & 1510 & 80.7 & 123 & .2 & 674 & .027 & $<.05$ & .77 & .92 & .78 & .84 & .83 & 13,300 \\
\hline $02-25-04$ & 1200 & 80.0 & 215 & .3 & 736 & E.065 & $<.60$ & .58 & .98 & .64 & .62 & .48 & 42,900 \\
\hline 08-26-04 & 1120 & 86.7 & 181 & .4 & 721 & $<.008$ & $<.06$ & .74 & .89 & .66 & .13 & $<.02$ & 3,730 \\
\hline 03-04-05 & 1155 & 81.3 & 234 & .4 & 766 & $<.008$ & $<.06$ & .65 & .89 & .59 & .06 & $<.02$ & 11,150 \\
\hline $08-04-05$ & 1440 & 75.0 & 232 & .5 & 788 & $<.008$ & $<.06$ & .66 & .94 & .67 & .28 & $<.02$ & 10,250 \\
\hline$*$ & 1455 & 74.4 & 230 & .5 & 783 & $<.008$ & $<.06$ & .65 & .94 & .66 & .22 & $<.02$ & 10,120 \\
\hline
\end{tabular}


Table 1. Values of physical properties and concentrations of inorganic constituents and nutrients in samples from wells and surface-water sites, 1999-2005.-Continued

$\left[\mu \mathrm{S} / \mathrm{cm}\right.$, microsiemens per centimeter at 25 degrees Celsius; $\mathrm{pH}$, in standard units; ${ }^{\circ} \mathrm{C}$, degrees Celsius; constituent concentrations are dissolved, unless otherwise noted; mg/L, milligrams per liter; $\mathrm{CaCO}_{3}$, calcium carbonate; ANC, total acid neutralizing capacity, incremental titration; N, nitrogen; $\mu \mathrm{g} / \mathrm{L}$, micrograms per liter; NGVD 29, National Geodetic Vertical Datum of 1929; --, no data; $<$, less than; E, estimated; *, replicate sample; >, greater than]

\begin{tabular}{|c|c|c|c|c|c|c|c|c|c|c|c|c|c|}
\hline Date & Time & $\begin{array}{c}\text { Waterlevel } \\
\text { (feet below } \\
\text { measuring } \\
\text { point) }\end{array}$ & $\begin{array}{c}\text { Specific } \\
\text { conductance } \\
(\mu \mathrm{S} / \mathrm{cm})\end{array}$ & pH & $\begin{array}{c}\text { Temperature } \\
\left({ }^{\circ} \mathrm{C}\right)\end{array}$ & $\begin{array}{c}\text { Dissolved } \\
\text { oxygen } \\
\text { (mg/L) }\end{array}$ & $\begin{array}{c}\text { Hardness } \\
\text { (mg/L as } \\
\mathrm{CaCO}_{3} \text { ) }\end{array}$ & $\begin{array}{c}\text { Calcium } \\
\text { (mg/L) }\end{array}$ & $\begin{array}{l}\text { Magnesium } \\
(\mathrm{mg} / \mathrm{L})\end{array}$ & $\begin{array}{l}\text { Sodium } \\
\text { (mg/L) }\end{array}$ & $\begin{array}{l}\text { Potassium } \\
\text { (mg/L) }\end{array}$ & $\begin{array}{l}\text { Alkalinity, } \\
\text { fixed } \\
\text { endpoint } \\
\text { (mg/L) }\end{array}$ & $\begin{array}{l}\text { ANC } \\
\text { (mg/L) }\end{array}$ \\
\hline \multicolumn{14}{|c|}{ USGS-1 (measuring point altitude 570.65 feet above NGVD 29) } \\
\hline 08-27-99 & 1010 & 10.99 & 736 & 7.2 & 14.1 & $<0.1$ & -- & 114 & 20.9 & 10.2 & 4.74 & 345 & 336 \\
\hline 02-29-00 & 1420 & 14.27 & 703 & 7.0 & 14.9 & $<.1$ & -- & 122 & 21.8 & 6.95 & 4.97 & 378 & 379 \\
\hline $08-23-00$ & 1250 & 13.86 & 811 & 7.2 & 16.2 & $<.1$ & -- & 125 & 21.9 & 6.03 & 5.26 & 291 & 374 \\
\hline 03-01-01 & 1130 & 11.22 & 827 & 7.1 & 14.5 & $<.1$ & -- & 128 & 21.8 & 7.09 & 5.11 & 367 & 373 \\
\hline $08-22-01$ & 1310 & 13.25 & 740 & 7.2 & 15.9 & $<.1$ & -- & 122 & 21.0 & 7.74 & 4.77 & 272 & 296 \\
\hline $02-25-02$ & 1530 & 15.90 & 884 & 7.1 & 14.3 & $<.1$ & -- & 135 & 23.3 & 8.78 & 5.04 & 347 & 337 \\
\hline $08-22-02$ & 1030 & 15.09 & 871 & 7.0 & 17.8 & $<.1$ & -- & 131 & 22.1 & 9.94 & 4.67 & 328 & 376 \\
\hline \multicolumn{14}{|c|}{ USGS-2S (measuring point altitude 572.68 feet above NGVD 29) } \\
\hline 08-26-99 & 1600 & 11.56 & 940 & 6.7 & 13.4 & $<.1$ & -- & 139 & 25.1 & 12.3 & 5.50 & 440 & 434 \\
\hline 02-29-00 & 0800 & 10.98 & 968 & 6.7 & 13.0 & $<.1$ & -- & 112 & 20.3 & 51.9 & 6.55 & 305 & 317 \\
\hline $08-23-00$ & 0850 & 13.63 & 1,010 & 7.1 & 14.0 & $<.1$ & -- & 123 & 22.1 & 45.8 & 6.34 & 372 & 373 \\
\hline $02-28-01$ & 1120 & 8.50 & 1,040 & 7.2 & 14.2 & $<.1$ & -- & 110 & 19.7 & 66.0 & 6.31 & 273 & 286 \\
\hline 08-21-01 & 1230 & 11.54 & 1,010 & 7.0 & 15.5 & $<.1$ & -- & 137 & 25.2 & 31.2 & 5.73 & 385 & 370 \\
\hline $02-26-02$ & 0840 & 14.11 & 1,070 & 7.0 & 13.5 & $<.1$ & -- & 130 & 22.6 & 49.8 & 5.98 & 339 & 344 \\
\hline \multicolumn{14}{|c|}{ USGS-2D (measuring point altitude 572.56 feet above NGVD 29) } \\
\hline 08-26-99 & 1510 & 11.42 & 1,030 & 6.7 & 13.4 & $<.1$ & -- & 102 & 24.6 & 44.5 & 5.95 & 250 & 244 \\
\hline 02-29-00 & 0850 & 10.86 & 1,020 & 6.7 & 12.5 & $<.1$ & -- & 97.9 & 23.3 & 85.3 & 6.41 & 298 & 302 \\
\hline $08-23-00$ & 0930 & 13.5 & 1,140 & 7.1 & 14.8 & $<.1$ & -- & 93.2 & 23.0 & 87.1 & 6.74 & 305 & 305 \\
\hline $02-28-01$ & 1100 & 8.67 & 1,200 & 7.3 & 13.3 & $<.1$ & -- & 107 & 24.5 & 86.3 & 6.53 & 283 & 285 \\
\hline 08-21-01 & 1320 & 11.43 & 1,180 & 7.0 & 15.9 & $<.1$ & -- & 97.6 & 23.5 & 93.9 & 6.09 & 243 & 272 \\
\hline $02-26-02$ & 0910 & 13.97 & 1,210 & 7.1 & 13.6 & $<.1$ & -- & 86.9 & 19.4 & 119 & 6.51 & 245 & 245 \\
\hline
\end{tabular}


$\left[\mu \mathrm{S} / \mathrm{cm}\right.$, microsiemens per centimeter at 25 degrees Celsius; $\mathrm{pH}$, in standard units; ${ }^{\circ} \mathrm{C}$, degrees Celsius; constituent concentrations are dissolved, unless otherwise noted; mg/L, milligrams per liter; $\mathrm{CaCO}_{3}$, calcium carbonate; ANC, total acid neutralizing capacity, incremental titration; N, nitrogen; $\mu \mathrm{g} / \mathrm{L}$, micrograms per liter; NGVD 29, National Geodetic Vertical Datum of 1929; --, no data; $<$, less than; E, estimated; *, replicate sample; >, greater than]

\begin{tabular}{|c|c|c|c|c|c|c|c|c|c|c|c|c|c|}
\hline Date & Time & $\begin{array}{l}\text { Sulfate } \\
\text { (mg/L) }\end{array}$ & $\begin{array}{c}\text { Chloride } \\
\text { (mg/L) }\end{array}$ & $\begin{array}{c}\text { Fluoride } \\
\text { (mg/L) }\end{array}$ & $\begin{array}{c}\text { Residue, sum } \\
\text { of } \\
\text { constituents } \\
(\mathrm{mg} / \mathrm{L}))\end{array}$ & $\begin{array}{c}\text { Nitrite as N } \\
(\mathrm{mg} / \mathrm{L})\end{array}$ & $\begin{array}{c}\text { Nitrite plus } \\
\text { nitrate as N } \\
\text { (mg/L) }\end{array}$ & $\begin{array}{c}\text { Ammonia as } \\
\mathbf{N} \\
(\mathbf{m g} / \mathrm{L})\end{array}$ & $\begin{array}{c}\text { Ammonia } \\
\text { plus organic } \\
\text { N, total } \\
(\mathrm{mg} / \mathrm{L})\end{array}$ & $\begin{array}{c}\text { Phosphorous, } \\
\text { total } \\
\text { (mg/L) }\end{array}$ & $\begin{array}{l}\text { Phosphorous, } \\
\text { dissolved } \\
\text { (mg/L) }\end{array}$ & $\begin{array}{c}\text { Ortho- } \\
\text { phosphorous } \\
\text { (mg/L) }\end{array}$ & $\begin{array}{c}\text { Iron } \\
(\mu \mathrm{g} / \mathrm{L})\end{array}$ \\
\hline \multicolumn{14}{|c|}{ USGS-1 } \\
\hline 08-27-99 & 1010 & 71.6 & 11.1 & 0.2 & -- & $<0.010$ & 0.14 & 0.16 & 0.26 & 0.16 & 0.14 & 0.08 & 3,930 \\
\hline $02-29-00$ & 1420 & 48.9 & 14.4 & .2 & -- & $<.010$ & $<.05$ & .15 & .25 & .12 & $<.05$ & .05 & 4,330 \\
\hline $08-23-00$ & 1250 & 50.0 & 21.9 & .2 & -- & $<.010$ & .07 & .13 & .22 & .10 & E.04 & .03 & 2,900 \\
\hline $03-01-01$ & 1130 & 53.6 & 35.0 & .3 & -- & $<.006$ & $<.05$ & .21 & .24 & E.04 & .13 & E.01 & 4,110 \\
\hline $08-22-01$ & 1310 & 56.2 & 19.0 & .2 & -- & $<.030$ & .28 & .91 & .26 & .13 & .13 & $<.09$ & 4,080 \\
\hline $02-25-02$ & 1530 & 53.7 & 58.5 & .3 & -- & E.007 & .05 & .17 & .23 & .13 & .11 & .10 & 4,500 \\
\hline $08-22-02$ & 1030 & 43.1 & 40.6 & .3 & -- & E.004 & .30 & .16 & .27 & .12 & .12 & .11 & 3,960 \\
\hline \multicolumn{14}{|c|}{ USGS-2S } \\
\hline 08-26-99 & 1600 & 48.9 & 26.2 & .1 & -- & .011 & $<.05$ & .65 & .78 & .85 & .53 & .64 & 21,700 \\
\hline $02-29-00$ & 0800 & 91.0 & 92.2 & .1 & -- & .029 & $<.05$ & .66 & .79 & .67 & $<.05$ & .32 & 17,800 \\
\hline $08-23-00$ & 0850 & 70.6 & 51.9 & .1 & -- & $<.010$ & $<.05$ & .60 & .78 & .69 & .67 & $<.01$ & 18,800 \\
\hline $02-28-01$ & 1120 & 133 & 94.2 & .2 & -- & E.004 & $<.05$ & .70 & 1.0 & .60 & .64 & $<.02$ & 16,500 \\
\hline $08-21-01$ & 1230 & 69.9 & 36.2 & .2 & -- & .021 & E.03 & .64 & .70 & .64 & .34 & E. 36 & 21,200 \\
\hline $02-26-02$ & 0840 & 99.4 & 87.2 & .2 & -- & .044 & $<.05$ & .80 & .87 & .67 & .57 & .27 & 19,500 \\
\hline \multicolumn{14}{|c|}{ USGS-2D } \\
\hline 08-26-99 & 1510 & 76.3 & 130 & .1 & -- & $<.010$ & $<.05$ & 1.20 & 1.5 & 1.13 & .49 & .18 & 23,000 \\
\hline $02-29-00$ & 0850 & 105 & 119 & .1 & -- & .049 & $<.05$ & 1.21 & 1.4 & 1.00 & $<.05$ & .80 & 21,400 \\
\hline $08-23-00$ & 0930 & 83.3 & 115 & .1 & -- & $<.010$ & $<.05$ & 1.14 & 1.4 & 1.02 & .74 & $<.01$ & 19,700 \\
\hline $02-28-01$ & 1100 & 129 & 137 & .2 & -- & $<.006$ & $<.05$ & 1.17 & 1.4 & .77 & .80 & $<.02$ & 22,100 \\
\hline $08-21-01$ & 1320 & 126 & 125 & .2 & -- & .094 & E. 12 & 6.34 & 1.5 & .88 & .30 & E. 17 & 20,200 \\
\hline $02-26-02$ & 0910 & 110 & 170 & .3 & -- & .058 & $<.05$ & 1.28 & 1.4 & .83 & .60 & .82 & 16,600 \\
\hline
\end{tabular}


Table 1. Values of physical properties and concentrations of inorganic constituents and nutrients in samples from wells and surface-water sites, 1999-2005.-Continued

$\left[\mu \mathrm{S} / \mathrm{cm}\right.$, microsiemens per centimeter at 25 degrees Celsius; $\mathrm{pH}$, in standard units; ${ }^{\circ} \mathrm{C}$, degrees Celsius; constituent concentrations are dissolved, unless otherwise noted; mg/L, milligrams per liter; $\mathrm{CaCO}_{3}$, calcium carbonate; ANC, total acid neutralizing capacity, incremental titration; N, nitrogen; $\mu \mathrm{g} / \mathrm{L}$, micrograms per liter; NGVD 29, National Geodetic Vertical Datum of 1929; --, no data; $<$, less than; E, estimated; *, replicate sample; >, greater than]

\begin{tabular}{|c|c|c|c|c|c|c|c|c|c|c|c|c|c|}
\hline Date & Time & $\begin{array}{c}\text { Waterlevel } \\
\text { (feet below } \\
\text { measuring } \\
\text { point) }\end{array}$ & $\begin{array}{c}\text { Specific } \\
\text { conductance } \\
(\mu \mathrm{S} / \mathrm{cm})\end{array}$ & pH & $\begin{array}{c}\text { Temperature } \\
\left({ }^{\circ} \mathrm{C}\right)\end{array}$ & $\begin{array}{c}\text { Dissolved } \\
\text { oxygen } \\
\text { (mg/L) }\end{array}$ & $\begin{array}{c}\text { Hardness } \\
\text { (mg/L as } \\
\mathrm{CaCO}_{3} \text { ) }\end{array}$ & $\begin{array}{c}\text { Calcium } \\
(\mathrm{mg} / \mathrm{L})\end{array}$ & $\begin{array}{l}\text { Magnesium } \\
(\mathbf{m g} / \mathbf{L})\end{array}$ & $\begin{array}{l}\text { Sodium } \\
\text { (mg/L) }\end{array}$ & $\begin{array}{l}\text { Potassium } \\
\text { (mg/L) }\end{array}$ & $\begin{array}{c}\text { Alkalinity, } \\
\text { fixed } \\
\text { endpoint } \\
\text { (mg/L) }\end{array}$ & $\begin{array}{l}\text { ANC } \\
\text { (mg/L) }\end{array}$ \\
\hline \multicolumn{14}{|c|}{ USGS-3S (measuring point altitude 570.28 feet above NGVD 29) } \\
\hline 08-26-99 & 1210 & 10.38 & 1,350 & 7.0 & 15.1 & $<0.1$ & -- & 119 & 19.1 & 119 & 6.14 & 279 & 272 \\
\hline 02-29-00 & 1000 & 9.46 & 1,420 & 6.8 & 14.0 & $<.1$ & -- & 132 & 24.5 & 146 & 6.24 & 329 & 333 \\
\hline $08-23-00$ & 1030 & 12.25 & 1,440 & 7.2 & 15.0 & $<.1$ & -- & 121 & 23.6 & 143 & 6.72 & 317 & 321 \\
\hline $02-27-01$ & 1350 & 3.97 & 1,130 & 7.2 & 11.9 & $<.1$ & -- & 107 & 20.4 & 115 & 4.88 & 320 & 320 \\
\hline 08-21-01 & 1430 & 10.55 & 1,060 & 7.2 & 14.3 & $<.1$ & -- & 82.3 & 15.5 & 106 & 4.46 & 241 & 240 \\
\hline $02-26-02$ & 1050 & 12.24 & 1,070 & 7.3 & 12.4 & $<.1$ & -- & 85.3 & 15.0 & 117 & 5.23 & 263 & 261 \\
\hline $08-22-02$ & 1200 & 13.03 & 1,180 & 7.3 & 15.3 & $<.1$ & -- & 93.7 & 15.4 & 123 & 5.14 & 282 & 256 \\
\hline 04-01-03 & 1320 & 14.23 & 1,050 & 7.2 & 12.5 & $<.1$ & -- & -- & -- & -- & -- & -- & -- \\
\hline \multicolumn{14}{|c|}{ USGS-3D (measuring point altitude 570.25 feet above NGVD 29) } \\
\hline 08-26-99 & 1050 & 10.59 & 963 & 6.9 & 15.1 & $<.1$ & -- & 129 & 31.5 & 19.5 & 4.92 & 489 & 476 \\
\hline $02-29-00$ & 1040 & 9.65 & 890 & 6.9 & 13.6 & $<.1$ & -- & 103 & 24.9 & 51.2 & 4.63 & 301 & 293 \\
\hline $08-23-00$ & 1110 & 12.42 & 1,000 & 7.2 & 14.3 & $<.1$ & -- & 121 & 29.8 & 27.5 & 5.05 & 316 & 315 \\
\hline $02-27-01$ & 1300 & 4.16 & 1,050 & 7.3 & 12.6 & $<.1$ & -- & 93.9 & 21.2 & 100 & 5.28 & 380 & 379 \\
\hline 08-21-01 & 1510 & 10.77 & 1,130 & 7.2 & 13.3 & $<.1$ & -- & 145 & 34.1 & 32.2 & 5.22 & 334 & 364 \\
\hline $02-26-02$ & 1020 & 12.49 & 1,050 & 7.3 & 11.6 & $<.1$ & -- & 103 & 22.8 & 75.4 & 5.21 & 304 & 305 \\
\hline \multicolumn{14}{|c|}{ USGS-4 (measuring point altitude 575.51 feet above NGVD 29) } \\
\hline $08-25-99$ & 1630 & 15.40 & 1,060 & 6.6 & 14.2 & $<.1$ & -- & 164 & 37.2 & 13.6 & 6.12 & 581 & 570 \\
\hline $03-01-00$ & 1000 & 16.75 & 885 & 6.9 & 13.6 & $<.1$ & -- & 121 & 29.8 & 11.8 & 5.63 & 432 & 436 \\
\hline $08-23-00$ & 1510 & 17.67 & 652 & 6.9 & 15.8 & $<.1$ & -- & 126 & 30.3 & 12.4 & 5.94 & 401 & 413 \\
\hline $02-22-01$ & 1440 & 16.91 & 955 & 7.0 & 13.6 & $<.1$ & -- & 128 & 29.6 & 16.0 & 5.19 & 322 & 338 \\
\hline 08-22-01 & 1420 & 16.83 & 963 & 7.1 & 15.4 & $<.1$ & -- & 136 & 31.2 & 15.0 & 5.63 & 327 & 376 \\
\hline $02-25-02$ & 1430 & 18.97 & 1,020 & 6.9 & 13.8 & $<.1$ & -- & 134 & 30.0 & 21.4 & 5.76 & 374 & 380 \\
\hline
\end{tabular}


$\left[\mu \mathrm{S} / \mathrm{cm}\right.$, microsiemens per centimeter at 25 degrees Celsius; $\mathrm{pH}$, in standard units; ${ }^{\circ} \mathrm{C}$, degrees Celsius; constituent concentrations are dissolved, unless otherwise noted; mg/L, milligrams per liter; $\mathrm{CaCO}_{3}$, calcium carbonate; ANC, total acid neutralizing capacity, incremental titration; N, nitrogen; $\mu \mathrm{g} / \mathrm{L}$, micrograms per liter; NGVD 29, National Geodetic Vertical Datum of 1929; --, no data; $<$, less than; E, estimated; *, replicate sample; >, greater than]

\begin{tabular}{|c|c|c|c|c|c|c|c|c|c|c|c|c|c|}
\hline Date & Time & $\begin{array}{l}\text { Sulfate } \\
\text { (mg/L) }\end{array}$ & $\begin{array}{c}\text { Chloride } \\
\text { (mg/L) }\end{array}$ & $\begin{array}{c}\text { Fluoride } \\
\text { (mg/L) }\end{array}$ & $\begin{array}{l}\text { Residue, sum } \\
\text { of } \\
\text { constituents } \\
(\mathrm{mg} / \mathrm{L}))\end{array}$ & $\begin{array}{l}\text { Nitrite as N } \\
\quad(\mathrm{mg} / \mathrm{L})\end{array}$ & $\begin{array}{c}\text { Nitrite plus } \\
\text { nitrate as N } \\
\text { (mg/L) }\end{array}$ & $\begin{array}{c}\text { Ammonia as } \\
\mathbf{N} \\
(\mathbf{m g} / \mathrm{L})\end{array}$ & $\begin{array}{c}\text { Ammonia } \\
\text { plus organic } \\
\text { N, total } \\
\text { (mg/L) }\end{array}$ & $\begin{array}{c}\text { Phosphorous, } \\
\text { total } \\
\text { (mg/L) }\end{array}$ & $\begin{array}{l}\text { Phosphorous, } \\
\text { dissolved } \\
\text { (mg/L) }\end{array}$ & $\begin{array}{c}\text { Ortho- } \\
\text { phosphorous } \\
\text { (mg/L) }\end{array}$ & $\begin{array}{l}\text { Iron } \\
(\mu \mathrm{g} / \mathrm{L})\end{array}$ \\
\hline \multicolumn{14}{|c|}{ USGS-3S } \\
\hline 08-26-99 & 1210 & 106 & 221 & 0.3 & -- & $<0.010$ & $<0.05$ & 0.17 & 0.01 & 0.41 & 0.22 & 0.07 & 8,820 \\
\hline $02-29-00$ & 1000 & 128 & 247 & .3 & -- & .021 & $<.05$ & .26 & .62 & .35 & $<.05$ & .16 & 11,200 \\
\hline $08-23-00$ & 1030 & 109 & 214 & .3 & -- & .012 & $<.05$ & .14 & .37 & .19 & .11 & .17 & 7,640 \\
\hline $02-27-01$ & 1350 & 130 & 136 & .3 & -- & E.003 & $<.05$ & .17 & .36 & .25 & .15 & $<.02$ & 8,270 \\
\hline 08-21-01 & 1430 & 80.1 & 139 & .3 & -- & E.004 & $<.05$ & E.19 & .36 & .28 & .14 & E.10 & 6,590 \\
\hline $02-26-02$ & 1050 & 136 & 113 & .4 & -- & .012 & $<.05$ & .20 & .41 & .29 & .20 & .27 & 5,370 \\
\hline $08-22-02$ & 1200 & 129 & 114 & .4 & -- & .009 & $<.05$ & .24 & .40 & .20 & .20 & .20 & 5,510 \\
\hline 04-01-03 & 1320 & -- & 90.5 & -- & -- & -- & -- & -- & -- & -- & -- & -- & -- \\
\hline \multicolumn{14}{|c|}{ USGS-3D } \\
\hline 08-26-99 & 1050 & 30.5 & 18.8 & .2 & -- & -- & -- & -- & -- & -- & -- & -- & 20,300 \\
\hline $02-29-00$ & 1040 & 90.2 & 84.1 & .2 & -- & .018 & $<.05$ & .60 & .79 & .59 & $<.05$ & .43 & 15,300 \\
\hline $08-23-00$ & 1110 & 82.9 & 71.5 & .2 & -- & $<.010$ & $<.05$ & .59 & .79 & .61 & .52 & $<.01$ & 18,000 \\
\hline $02-27-01$ & 1300 & 70.5 & 102 & .3 & -- & $<.006$ & $<.05$ & .73 & .96 & .60 & .45 & E.02 & 12,700 \\
\hline 08-21-01 & 1510 & 85.5 & 94.5 & .2 & -- & .017 & E.02 & .76 & .91 & .52 & E.61 & E.09 & 19,300 \\
\hline $02-26-02$ & 1020 & 100 & 104 & .3 & -- & .037 & $<.05$ & .84 & .96 & .52 & .41 & $<.09$ & 13,200 \\
\hline \multicolumn{14}{|c|}{ USGS-4 } \\
\hline $08-25-99$ & 1630 & 24.4 & 9.3 & .2 & -- & $<.010$ & $<.05$ & .43 & .61 & .46 & .36 & .30 & 13,800 \\
\hline 03-01-00 & 1000 & 40.9 & 26.5 & .1 & -- & .018 & $<.05$ & .37 & .47 & .41 & .14 & .23 & 10,800 \\
\hline $08-23-00$ & 1510 & 40.5 & 40.1 & .2 & -- & .01 & $<.05$ & .31 & .43 & .41 & .19 & .07 & 11,300 \\
\hline $02-22-01$ & 1440 & 77.7 & 73.1 & .2 & -- & $<.006$ & $<.05$ & .34 & .45 & .39 & .17 & .04 & 11,400 \\
\hline $08-22-01$ & 1420 & 72.9 & 59.1 & E. 2 & -- & $<.010$ & $<.09$ & .67 & .44 & .39 & E.43 & E.07 & 12,400 \\
\hline $02-25-02$ & 1430 & 77.6 & 67.5 & .2 & -- & .032 & $<.05$ & .31 & .44 & .42 & .36 & .32 & 12,200 \\
\hline
\end{tabular}


Table 1. Values of physical properties and concentrations of inorganic constituents and nutrients in samples from wells and surface-water sites, 1999-2005.-Continued

$\left[\mu \mathrm{S} / \mathrm{cm}\right.$, microsiemens per centimeter at 25 degrees Celsius; $\mathrm{pH}$, in standard units; ${ }^{\circ} \mathrm{C}$, degrees Celsius; constituent concentrations are dissolved, unless otherwise noted; mg/L, milligrams per liter; $\mathrm{CaCO}_{3}$, calcium carbonate; ANC, total acid neutralizing capacity, incremental titration; N, nitrogen; $\mu \mathrm{g} / \mathrm{L}$, micrograms per liter; NGVD 29, National Geodetic Vertical Datum of 1929; --, no data; $<$, less than; E, estimated; *, replicate sample; >, greater than]

\begin{tabular}{|c|c|c|c|c|c|c|c|c|c|c|c|c|c|}
\hline Date & Time & $\begin{array}{c}\text { Waterlevel } \\
\text { (feet below } \\
\text { measuring } \\
\text { point) }\end{array}$ & $\begin{array}{c}\text { Specific } \\
\text { conductance } \\
(\mu \mathrm{S} / \mathrm{cm})\end{array}$ & pH & $\begin{array}{c}\text { Temperature } \\
\left({ }^{\circ} \mathrm{C}\right)\end{array}$ & $\begin{array}{c}\text { Dissolved } \\
\text { oxygen } \\
\text { (mg/L) }\end{array}$ & $\begin{array}{c}\text { Hardness } \\
(\mathrm{mg} / \mathrm{L} \text { as } \\
\left.\mathrm{CaCO}_{3}\right)\end{array}$ & $\begin{array}{c}\text { Calcium } \\
\text { (mg/L) }\end{array}$ & $\begin{array}{l}\text { Magnesium } \\
(\mathrm{mg} / \mathrm{L})\end{array}$ & $\begin{array}{l}\text { Sodium } \\
\text { (mg/L) }\end{array}$ & $\begin{array}{l}\text { Potassium } \\
\text { (mg/L) }\end{array}$ & $\begin{array}{c}\text { Alkalinity, } \\
\text { fixed } \\
\text { endpoint } \\
\text { (mg/L) }\end{array}$ & $\begin{array}{l}\text { ANC } \\
\text { (mg/L) }\end{array}$ \\
\hline \multicolumn{14}{|c|}{ USGS-5S (measuring point altitude 571.98 feet above NGVD 29) } \\
\hline 08-31-99 & 1010 & -- & 898 & 7.0 & 14.4 & $<0.1$ & -- & 137 & 29.5 & 11.8 & 5.00 & 430 & 437 \\
\hline 03-01-00 & 1130 & 9.06 & 1,020 & 6.9 & 13.0 & $<.1$ & -- & -- & -- & -- & -- & -- & -- \\
\hline $08-24-00$ & 0850 & 12.78 & 883 & 7.0 & 15.3 & $<.1$ & -- & 131 & 26.2 & 12.0 & 4.79 & 406 & 412 \\
\hline $02-22-01$ & 0900 & 6.81 & 977 & 6.9 & 13.4 & $<.1$ & -- & 147 & 28.7 & 12.9 & 4.72 & 382 & 382 \\
\hline $08-22-01$ & 1530 & 10.10 & 966 & 7.1 & 15.2 & $<.1$ & -- & 150 & 29.2 & 11.9 & 4.82 & 317 & 306 \\
\hline $02-27-02$ & 1240 & 12.31 & 982 & 7.0 & 13.5 & $<.1$ & -- & 154 & 27.9 & 11.6 & 4.78 & 400 & 399 \\
\hline 08-19-02 & 1420 & 12.62 & 953 & 7.0 & 15.1 & $<.1$ & -- & 146 & 26.4 & 13.0 & 4.69 & 347 & 252 \\
\hline 04-01-03 & 1540 & 13.61 & 955 & 6.9 & 13.9 & $<.1$ & -- & -- & -- & -- & -- & -- & -- \\
\hline \multicolumn{14}{|c|}{ USGS-5D (measuring point altitude 571.94 feet above NGVD 29) } \\
\hline 08-31-99 & 0920 & -- & 1,100 & 7.1 & 14.4 & $<.1$ & -- & 141 & 28.2 & 34.3 & 5.83 & 319 & 320 \\
\hline 03-01-00 & 1200 & 9.02 & 941 & 6.8 & 13.3 & $<.1$ & -- & -- & -- & -- & -- & -- & -- \\
\hline $08-24-00$ & 0940 & 12.74 & 1,040 & 7.1 & 15.1 & $<.1$ & -- & 113 & 24.6 & 55.0 & 5.24 & 289 & 291 \\
\hline $02-22-01$ & 0940 & 6.78 & 1,170 & 7.1 & 13.6 & $<.1$ & -- & 108 & 22.6 & 87.3 & 5.20 & 234 & 234 \\
\hline $08-22-01$ & 1610 & 10.06 & 1,130 & 7.3 & 15.0 & $<.1$ & -- & 99.0 & 21.7 & 94.7 & 4.83 & 263 & 260 \\
\hline $02-27-02$ & 1330 & 12.28 & 985 & 7.3 & 13.1 & $<.1$ & -- & 78.2 & 15.8 & 98.4 & 4.98 & 275 & 274 \\
\hline 08-19-02 & 1500 & 12.56 & 1,190 & 7.1 & 15.5 & $<.1$ & -- & 112 & 21.6 & 89.7 & 5.93 & 267 & 225 \\
\hline \multicolumn{14}{|c|}{ USGS-6 (measuring point altitude 570.71 feet above NGVD 29) } \\
\hline 08-31-99 & 0810 & -- & 1,250 & 7.0 & 13.6 & $<.1$ & -- & 122 & 27.3 & 87.7 & 6.02 & 303 & 303 \\
\hline $03-02-00$ & 1030 & 13.61 & 1,240 & 6.5 & 14.4 & $<.1$ & -- & 117 & 25.8 & 97.8 & 7.00 & 339 & 344 \\
\hline $08-23-00$ & 1610 & 14.11 & 1,360 & 7.0 & 14.8 & $<.1$ & -- & 123 & 27.3 & 90.3 & 7.00 & 312 & 325 \\
\hline $02-22-01$ & 1040 & 11.10 & 1,270 & 6.9 & 14.8 & $<.1$ & -- & 115 & 25.2 & 103 & 6.32 & 335 & 334 \\
\hline 08-24-01 & 0900 & 14.46 & 1,340 & 7.0 & 14.1 & $<.1$ & -- & 114 & 25.6 & 108 & 6.09 & 287 & 281 \\
\hline $02-25-02$ & 1620 & 15.46 & 1,270 & 6.9 & 14.7 & $<.1$ & -- & 116 & 24.8 & 103 & 6.25 & 364 & 367 \\
\hline 08-19-02 & 1300 & 14.25 & 1,340 & 7.0 & 14.6 & $<.1$ & -- & 117 & 25.2 & 109 & 5.99 & 323 & 277 \\
\hline 04-01-03 & 1440 & 16.08 & 1,190 & 7.0 & 14.9 & $<.1$ & -- & -- & -- & -- & -- & -- & -- \\
\hline
\end{tabular}


$\left[\mu \mathrm{S} / \mathrm{cm}\right.$, microsiemens per centimeter at 25 degrees Celsius; $\mathrm{pH}$, in standard units; ${ }^{\circ} \mathrm{C}$, degrees Celsius; constituent concentrations are dissolved, unless otherwise noted; mg/L, milligrams per liter; $\mathrm{CaCO}_{3}$, calcium carbonate; ANC, total acid neutralizing capacity, incremental titration; N, nitrogen; $\mu \mathrm{g} / \mathrm{L}$, micrograms per liter; NGVD 29, National Geodetic Vertical Datum of 1929; --, no data; $<$, less than; E, estimated; *, replicate sample; >, greater than]

\begin{tabular}{|c|c|c|c|c|c|c|c|c|c|c|c|c|c|}
\hline Date & Time & $\begin{array}{l}\text { Sulfate } \\
\text { (mg/L) }\end{array}$ & $\begin{array}{c}\text { Chloride } \\
\text { (mg/L) }\end{array}$ & $\begin{array}{c}\begin{array}{c}\text { Fluoride } \\
\text { (mg/L) }\end{array} \\
\end{array}$ & $\begin{array}{c}\text { Residue, sum } \\
\text { of } \\
\text { constituents } \\
\text { (mg/L)) }\end{array}$ & $\begin{array}{c}\text { Nitrite as N } \\
\quad(\mathrm{mg} / \mathrm{L})\end{array}$ & $\begin{array}{c}\text { Nitrite plus } \\
\text { nitrate as N } \\
\quad(\mathrm{mg} / \mathrm{L})\end{array}$ & $\begin{array}{c}\text { Ammonia as } \\
\mathbf{N} \\
(\mathbf{m g} / \mathrm{L})\end{array}$ & $\begin{array}{c}\text { Ammonia } \\
\text { plus organic } \\
\mathrm{N}, \text { total } \\
(\mathrm{mg} / \mathrm{L})\end{array}$ & $\begin{array}{c}\text { Phosphorous, } \\
\text { total } \\
\text { (mg/L) }\end{array}$ & $\begin{array}{c}\text { Phosphorous, } \\
\text { dissolved } \\
\text { (mg/L) }\end{array}$ & $\begin{array}{c}\text { Ortho- } \\
\text { phosphorous } \\
\text { (mg/L) }\end{array}$ & $\begin{array}{c}\text { Iron } \\
(\mu \mathrm{g} / \mathrm{L})\end{array}$ \\
\hline \multicolumn{14}{|c|}{ USGS-5S } \\
\hline 08-31-99 & 1010 & 39.2 & 33.4 & 0.2 & -- & $<0.010$ & $<0.05$ & 0.04 & E0.09 & $<0.05$ & $<0.05$ & $<0.01$ & 19 \\
\hline $03-01-00$ & 1130 & 141 & 108 & .2 & -- & $<.010$ & $<.05$ & .03 & E.10 & $<.05$ & $<.05$ & $<.01$ & 15 \\
\hline $08-24-00$ & 0850 & 45.5 & 40.7 & .2 & -- & $<.010$ & $<.05$ & .04 & .13 & $<.05$ & $<.05$ & .01 & 22 \\
\hline $02-22-01$ & 0900 & 57.8 & 62.9 & .2 & -- & $<.006$ & $<.05$ & E.03 & .11 & $<.06$ & $<.06$ & E.01 & 22 \\
\hline $08-22-01$ & 1530 & 67.6 & 76.8 & .2 & -- & $<.006$ & $<.05$ & .04 & .13 & $<.06$ & $<.06$ & $<.09$ & 38 \\
\hline $02-27-02$ & 1240 & 69.5 & 65.0 & .2 & -- & $<.008$ & $<.05$ & E.03 & .11 & $<.06$ & $<.06$ & E.01 & 26 \\
\hline 08-19-02 & 1420 & 56.8 & 60.4 & .2 & -- & $<.008$ & $<.05$ & E.04 & .10 & $<.06$ & $<.06$ & E.01 & 35 \\
\hline 04-01-03 & 1540 & -- & 54.6 & -- & -- & & & & & & & & \\
\hline \multicolumn{14}{|c|}{ USGS-5D } \\
\hline 08-31-99 & 0920 & 82.7 & 119 & .2 & -- & 0.02 & $<.05$ & .81 & .92 & .47 & .43 & .52 & 15,800 \\
\hline 03-01-00 & 1200 & 60.7 & 77.3 & .1 & -- & .019 & $<.05$ & .65 & .81 & 0.42 & $<.05$ & .27 & 10,400 \\
\hline $08-24-00$ & 0940 & 84 & 104 & .2 & -- & .025 & $<.05$ & .64 & .76 & .47 & .39 & .37 & 13,800 \\
\hline $02-22-01$ & 0940 & 114 & 167 & .3 & -- & E.005 & $<.05$ & .67 & .79 & .38 & .11 & $<.02$ & 13,000 \\
\hline $08-22-01$ & 1610 & 122 & 134 & .2 & -- & .014 & E.03 & .71 & .88 & .44 & .38 & E.08 & 12,000 \\
\hline $02-27-02$ & 1330 & 82.6 & 106 & .3 & -- & .016 & $<.05$ & .67 & .80 & .47 & .46 & .47 & 8,980 \\
\hline 08-19-02 & 1500 & 112 & 145 & .2 & -- & .032 & $<.05$ & .78 & .91 & .48 & .49 & .52 & 12,000 \\
\hline \multicolumn{14}{|c|}{ USGS-6 } \\
\hline 08-31-99 & 0810 & 93.6 & 179 & .2 & -- & .026 & $<.05$ & .90 & .93 & .61 & .74 & .55 & 17,100 \\
\hline $03-02-00$ & 1030 & 150 & 131 & .2 & -- & .023 & $<.05$ & .88 & 1.2 & .73 & .10 & .58 & 15,800 \\
\hline $08-23-00$ & 1610 & 113 & 176 & .2 & -- & $<.010$ & $<.05$ & .94 & 1.2 & .71 & .09 & .04 & 16,500 \\
\hline $02-22-01$ & 1040 & 135 & 136 & .3 & -- & .011 & $<.05$ & 1.01 & 1.2 & .66 & .59 & $<.02$ & 14,800 \\
\hline 08-24-01 & 0900 & 105 & 183 & .2 & -- & .018 & E.03 & 1.01 & 1.3 & .64 & E. 57 & E. 12 & 14,700 \\
\hline $02-25-02$ & 1620 & 130 & 118 & .3 & -- & .024 & $<.05$ & .91 & 1.3 & .68 & .78 & .59 & 14,800 \\
\hline 08-19-02 & 1300 & 110 & 169 & .2 & -- & .030 & $<.05$ & 1.13 & 1.4 & .73 & .70 & .60 & 14,300 \\
\hline 04-01-03 & 1440 & -- & 121 & -- & -- & -- & -- & -- & -- & -- & -- & -- & -- \\
\hline
\end{tabular}


Table 1. Values of physical properties and concentrations of inorganic constituents and nutrients in samples from wells and surface-water sites, 1999-2005.-Continued

$\left[\mu \mathrm{S} / \mathrm{cm}\right.$, microsiemens per centimeter at 25 degrees Celsius; $\mathrm{pH}$, in standard units; ${ }^{\circ} \mathrm{C}$, degrees Celsius; constituent concentrations are dissolved, unless otherwise noted; mg/L, milligrams per liter; $\mathrm{CaCO}_{3}$, calcium carbonate; ANC, total acid neutralizing capacity, incremental titration; N, nitrogen; $\mu \mathrm{g} / \mathrm{L}$, micrograms per liter; NGVD 29, National Geodetic Vertical Datum of 1929; --, no data; $<$, less than; E, estimated; *, replicate sample; >, greater than]

\begin{tabular}{|c|c|c|c|c|c|c|c|c|c|c|c|c|c|}
\hline Date & Time & $\begin{array}{c}\text { Waterlevel } \\
\text { (feet below } \\
\text { measuring } \\
\text { point) }\end{array}$ & $\begin{array}{c}\text { Specific } \\
\text { conductance } \\
(\mu \mathrm{S} / \mathrm{cm})\end{array}$ & $\mathrm{pH}$ & $\begin{array}{c}\text { Temperature } \\
\left({ }^{\circ} \mathrm{C}\right)\end{array}$ & $\begin{array}{c}\text { Dissolved } \\
\text { oxygen } \\
\text { (mg/L) }\end{array}$ & $\begin{array}{c}\text { Hardness } \\
\text { (mg/L as } \\
\mathrm{CaCO}_{3} \text { ) }\end{array}$ & $\begin{array}{c}\text { Calcium } \\
\text { (mg/L) }\end{array}$ & $\begin{array}{l}\text { Magnesium } \\
\quad(\mathrm{mg} / \mathrm{L})\end{array}$ & $\begin{array}{l}\text { Sodium } \\
\text { (mg/L) }\end{array}$ & $\begin{array}{l}\text { Potassium } \\
\text { (mg/L) }\end{array}$ & $\begin{array}{l}\text { Alkalinity, } \\
\text { fixed } \\
\text { endpoint } \\
\text { (mg/L) }\end{array}$ & $\begin{array}{l}\text { ANC } \\
\text { (mg/L) }\end{array}$ \\
\hline \multicolumn{14}{|c|}{ USGS-7 (measuring point altitude 571.28 feet above NGVD 29) } \\
\hline $08-25-99$ & 1510 & 13.33 & 1,130 & 6.7 & 9.7 & $<0.1$ & -- & 85.0 & 19.2 & 111 & 5.03 & 211 & 204 \\
\hline * & 1515 & 13.33 & 1,130 & 6.7 & 9.7 & $<.1$ & -- & 84.4 & 19.3 & 111 & 4.96 & 210 & 205 \\
\hline $03-1-00$ & 0850 & 15.92 & 1,000 & 7.1 & 9.5 & $<.1$ & -- & 67.5 & 15.9 & 120 & 4.38 & 213 & 215 \\
\hline$*$ & 0855 & 15.92 & 1,000 & 7.1 & 9.5 & $<.1$ & -- & 67.3 & 15.9 & 119 & 4.41 & 212 & 214 \\
\hline $08-23-00$ & 1400 & 16.03 & 925 & 7.4 & 13.6 & $<.1$ & -- & 63.9 & 14.2 & 100 & 4.91 & 181 & 181 \\
\hline * & 1405 & 16.03 & 925 & 7.4 & 13.6 & $<.1$ & -- & 62.1 & 14.2 & 101 & 4.97 & 178 & 180 \\
\hline $02-22-01$ & 1050 & 15.28 & 1,010 & 7.3 & 11.0 & $<.1$ & -- & 69.2 & 14.3 & 109 & 4.41 & 200 & 202 \\
\hline * & 1055 & 15.28 & 1,010 & 7.3 & 11.0 & $<.1$ & -- & 69.2 & 14.4 & 109 & 4.43 & 201 & 201 \\
\hline 08-21-01 & 1630 & 15.91 & 998 & 7.3 & 14.3 & $<.1$ & -- & 73.0 & 15.2 & 111 & 5.10 & 205 & 202 \\
\hline * & 1635 & 15.91 & 998 & 7.3 & 14.3 & $<.1$ & -- & 69.3 & 15.2 & 108 & 5.05 & 206 & 202 \\
\hline $02-25-02$ & 1320 & 17.38 & 1,010 & 7.4 & 10.8 & $<.1$ & -- & 78.0 & 15.3 & 104 & 5.15 & 227 & 224 \\
\hline * & 1325 & 17.38 & 1,010 & 7.4 & 10.8 & $<.1$ & -- & 77.3 & 15.3 & 104 & 5.02 & 224 & 226 \\
\hline \multicolumn{14}{|c|}{ USGS-8S (measuring point altitude 574.68 feet above NGVD 29) } \\
\hline 06-04-99 & 0830 & 9.69 & 780 & 6.9 & 14.9 & 0.2 & -- & 117 & 23.2 & 5.07 & 8.05 & -- & 384 \\
\hline 09-01-99 & 1000 & -- & 788 & 7.0 & 14.7 & $<.1$ & -- & 126 & 25.3 & 5.19 & 8.52 & 411 & 410 \\
\hline 03-08-00 & 0810 & 24.42 & 714 & 6.9 & 15.3 & $<.1$ & -- & 113 & 21.3 & 4.87 & 5.70 & 365 & 363 \\
\hline 08-29-00 & 1230 & 20.72 & 749 & 7.0 & 16.7 & 0.2 & -- & 124 & 23.1 & 5.38 & 5.65 & 370 & 369 \\
\hline 02-21-01 & 1440 & 22.91 & 791 & 6.9 & 13.9 & 0.2 & -- & 126 & 24.2 & 4.95 & 5.71 & 383 & 382 \\
\hline 08-23-01 & 1330 & 20.68 & 821 & 7.1 & 15.6 & 0.4 & -- & 135 & 26.0 & 5.26 & 6.63 & 343 & 344 \\
\hline $02-27-02$ & 1430 & 22.57 & 751 & 7.2 & 14.2 & $<.1$ & -- & 121 & 22.5 & 4.62 & 5.69 & 362 & 376 \\
\hline 08-19-02 & 1610 & 23.15 & 836 & 7.0 & 16.2 & 0.9 & -- & 139 & 24.4 & 5.21 & 6.40 & 407 & 354 \\
\hline \multicolumn{14}{|c|}{ USGS-8D (measuring point altitude 574.51 feet above NGVD 29) } \\
\hline 06-04-99 & 0920 & 9.85 & 815 & 7.0 & 16 & $<.1$ & -- & 119 & 26.4 & 9.77 & 6.75 & -- & 424 \\
\hline 09-01-99 & 1050 & -- & 804 & 7.1 & 14.8 & $<.1$ & -- & 119 & 25.9 & 12.1 & 6.78 & 425 & 423 \\
\hline $03-08-00$ & 0840 & 24.88 & 765 & 7.0 & 15.3 & $<.1$ & -- & 111 & 24.2 & 11.1 & 7.24 & 398 & 400 \\
\hline $08-29-00$ & 1150 & 20.57 & 770 & 7.1 & 16.2 & $<.1$ & -- & 116 & 25.5 & 14.8 & 6.98 & 401 & 402 \\
\hline 02-21-01 & 1530 & 22.98 & 796 & 7.0 & 13.8 & $<.1$ & -- & 117 & 25.3 & 13.2 & 6.81 & 398 & 397 \\
\hline 08-23-01 & 1420 & 21.35 & 814 & 7.3 & 15.7 & $<.1$ & -- & 117 & 25.1 & 14.6 & 7.45 & 350 & 319 \\
\hline $02-27-02$ & 1510 & 22.40 & 749 & 7.2 & 13.7 & $<.1$ & -- & 109 & 22.7 & 14.3 & 6.53 & 379 & 364 \\
\hline 08-19-02 & 1650 & 23.51 & 782 & 7.1 & 16.6 & $<.1$ & -- & 115 & 23.8 & 14.0 & 6.94 & 365 & 315 \\
\hline
\end{tabular}


$\left[\mu \mathrm{S} / \mathrm{cm}\right.$, microsiemens per centimeter at 25 degrees Celsius; $\mathrm{pH}$, in standard units; ${ }^{\circ} \mathrm{C}$, degrees Celsius; constituent concentrations are dissolved, unless otherwise noted; mg/L, milligrams per liter; $\mathrm{CaCO}_{3}$, calcium carbonate; ANC, total acid neutralizing capacity, incremental titration; N, nitrogen; $\mu \mathrm{g} / \mathrm{L}$, micrograms per liter; NGVD 29, National Geodetic Vertical Datum of 1929; --, no data; $<$, less than; E, estimated; *, replicate sample; >, greater than]

\begin{tabular}{|c|c|c|c|c|c|c|c|c|c|c|c|c|c|}
\hline Date & Time & $\begin{array}{c}\text { Sulfate } \\
\text { (mg/L) }\end{array}$ & $\begin{array}{c}\text { Chloride } \\
\text { (mg/L) }\end{array}$ & $\begin{array}{c}\text { Fluoride } \\
\text { (mg/L) }\end{array}$ & $\begin{array}{c}\text { Residue, sum } \\
\text { of } \\
\text { constituents } \\
(\mathrm{mg} / \mathrm{L}))\end{array}$ & $\begin{array}{l}\text { Nitrite as N } \\
(\mathrm{mg} / \mathrm{L})\end{array}$ & $\begin{array}{c}\text { Nitrite plus } \\
\text { nitrate as N } \\
\text { (mg/L) }\end{array}$ & $\begin{array}{c}\text { Ammonia as } \\
\mathbf{N} \\
(\mathrm{mg} / \mathrm{L})\end{array}$ & $\begin{array}{c}\text { Ammonia } \\
\text { plus organic } \\
\mathrm{N}, \text { total } \\
(\mathrm{mg} / \mathrm{L})\end{array}$ & $\begin{array}{c}\text { Phosphorous, } \\
\text { total } \\
\text { (mg/L) }\end{array}$ & $\begin{array}{c}\text { Phosphorous, } \\
\text { dissolved } \\
\text { (mg/L) }\end{array}$ & $\begin{array}{c}\text { Ortho- } \\
\text { phosphorous } \\
\text { (mg/L) }\end{array}$ & $\begin{array}{c}\text { Iron } \\
(\mu \mathrm{g} / \mathrm{L})\end{array}$ \\
\hline \multicolumn{14}{|c|}{ USGS-7 } \\
\hline 08-25-99 & 1510 & 116 & 157 & 0.3 & -- & $<0.010$ & $<0.05$ & 0.77 & 1.1 & 0.37 & 0.37 & 0.23 & 6,120 \\
\hline$*$ & 1515 & 115 & 155 & .3 & -- & -- & -- & -- & -- & -- & -- & -- & 6,130 \\
\hline $03-01-00$ & 0850 & 180 & 89.9 & .3 & -- & $<.010$ & $<.05$ & .53 & .77 & .28 & $<.05$ & .04 & 4,970 \\
\hline$*$ & 0855 & 181 & 90.3 & .3 & -- & $<.010$ & $<.05$ & .52 & .74 & .30 & $<.05$ & .08 & 4,950 \\
\hline $08-23-00$ & 1400 & 161 & 85.3 & .4 & -- & $<.010$ & $<.05$ & .49 & .70 & .29 & .16 & .23 & 4,460 \\
\hline$*$ & 1405 & 151 & 77.0 & .4 & -- & $<.010$ & $<.05$ & .48 & .72 & .29 & .21 & .25 & 4,430 \\
\hline $02-22-01$ & 1050 & 156 & 99.5 & .6 & -- & E.003 & $<.05$ & .37 & .55 & .24 & .14 & $<.02$ & 4,680 \\
\hline * & 1055 & 156 & 98.9 & .6 & -- & E.004 & $<.05$ & .37 & .56 & .24 & .16 & $<.02$ & 4,690 \\
\hline $08-21-01$ & 1630 & 156 & 99.8 & .5 & -- & $<.006$ & $<.05$ & E. 46 & .72 & .17 & .11 & E. 12 & 2,650 \\
\hline$*$ & 1635 & 156 & 101 & .5 & -- & $<.006$ & $<.05$ & E. 44 & .66 & .18 & .12 & E.11 & 2,570 \\
\hline $02-25-02$ & 1320 & 154 & 91.6 & .5 & -- & .008 & $<.05$ & .31 & .43 & .23 & .19 & .23 & 5,090 \\
\hline * & 1325 & 154 & 91.7 & .5 & -- & .009 & $<.05$ & .35 & .48 & .26 & .22 & .26 & 5,080 \\
\hline \multicolumn{14}{|c|}{ USGS-8S } \\
\hline 06-04-99 & 0830 & 37.8 & 6.36 & .3 & -- & .049 & 2.33 & .06 & .12 & $<.05$ & $<.05$ & .02 & $<10$ \\
\hline 09-01-99 & 1000 & 34.7 & 7.22 & .3 & -- & .018 & .78 & $<.02$ & E.09 & E.04 & $<.05$ & $<.01$ & $<10$ \\
\hline $03-08-00$ & 0810 & 42.4 & 6.20 & .2 & -- & .064 & 3.30 & .02 & .15 & $<.05$ & $<.05$ & $<.01$ & 16 \\
\hline $08-29-00$ & 1230 & 56.5 & 4.56 & .2 & -- & .052 & 1.79 & $<.02$ & E.07 & $<.05$ & $<.05$ & $<.01$ & $<10$ \\
\hline $02-21-01$ & 1440 & 42.9 & 4.03 & .3 & -- & .053 & 2.47 & $<.04$ & .09 & $<.06$ & $<.06$ & $<.02$ & $<10$ \\
\hline 08-23-01 & 1330 & 43.2 & 4.79 & .3 & -- & .021 & .98 & E.02 & .10 & $<.06$ & $<.06$ & E.03 & $<10$ \\
\hline $02-27-02$ & 1430 & 40.5 & 4.26 & .3 & -- & .066 & 4.93 & $<.04$ & .13 & E.03 & $<.06$ & $<.09$ & $<10$ \\
\hline 08-19-02 & 1610 & 30.9 & 3.71 & .3 & -- & .033 & 1.99 & $<.04$ & E. 10 & $<.06$ & $<.06$ & E.01 & $<10$ \\
\hline \multicolumn{14}{|c|}{ USGS-8D } \\
\hline 06-04-99 & 0920 & 35.6 & 5.30 & .2 & -- & .021 & $<.05$ & .20 & .24 & .41 & .39 & .37 & 4,920 \\
\hline 09-01-99 & 1050 & 19.2 & 10.1 & .3 & -- & .01 & $<.05$ & .12 & .19 & .36 & .31 & .42 & 7,930 \\
\hline $03-08-00$ & 0840 & 36.9 & 7.23 & .3 & -- & $<.010$ & $<.05$ & .11 & .22 & .30 & E.05 & .06 & 7,170 \\
\hline $08-29-00$ & 1150 & 32.8 & 8.46 & .3 & -- & .014 & $<.05$ & .14 & .27 & .46 & E.04 & .28 & 8,470 \\
\hline $02-21-01$ & 1530 & 34.6 & 7.26 & .3 & -- & E.003 & $<.05$ & .10 & .20 & .39 & .21 & E.01 & 8,080 \\
\hline $08-23-01$ & 1420 & 24.8 & 9.73 & .3 & -- & .008 & E.03 & .12 & .23 & .38 & E.34 & E.06 & 7,850 \\
\hline $02-27-02$ & 1510 & 36.7 & 9.91 & .3 & -- & .017 & $<.05$ & .18 & .25 & .44 & .39 & .41 & 8,340 \\
\hline 08-19-02 & 1650 & 31.9 & 7.92 & .3 & -- & .012 & $<.05$ & .15 & .23 & .41 & .43 & .45 & 7,660 \\
\hline
\end{tabular}


Table 1. Values of physical properties and concentrations of inorganic constituents and nutrients in samples from wells and surface-water sites, 1999-2005.-Continued

$\left[\mu \mathrm{S} / \mathrm{cm}\right.$, microsiemens per centimeter at 25 degrees Celsius; $\mathrm{pH}$, in standard units; ${ }^{\circ} \mathrm{C}$, degrees Celsius; constituent concentrations are dissolved, unless otherwise noted; mg/L, milligrams per liter; $\mathrm{CaCO}_{3}$, calcium carbonate; ANC, total acid neutralizing capacity, incremental titration; N, nitrogen; $\mu \mathrm{g} / \mathrm{L}$, micrograms per liter; NGVD 29, National Geodetic Vertical Datum of 1929; --, no data; $<$, less than; E, estimated; *, replicate sample; >, greater than]

\begin{tabular}{|c|c|c|c|c|c|c|c|c|c|c|c|c|c|}
\hline Date & Time & $\begin{array}{c}\text { Waterlevel } \\
\text { (feet below } \\
\text { measuring } \\
\text { point) }\end{array}$ & $\begin{array}{c}\text { Specific } \\
\text { conductance } \\
(\mu \mathrm{S} / \mathrm{cm})\end{array}$ & pH & $\begin{array}{c}\text { Temperature } \\
\left({ }^{\circ} \mathrm{C}\right)\end{array}$ & $\begin{array}{c}\text { Dissolved } \\
\text { oxygen } \\
\text { (mg/L) }\end{array}$ & $\begin{array}{c}\text { Hardness } \\
\text { (mg/L as } \\
\mathrm{CaCO}_{3} \text { ) }\end{array}$ & $\begin{array}{c}\text { Calcium } \\
\text { (mg/L) }\end{array}$ & $\begin{array}{l}\text { Magnesium } \\
(\mathrm{mg} / \mathrm{L})\end{array}$ & $\begin{array}{l}\text { Sodium } \\
\text { (mg/L) }\end{array}$ & $\begin{array}{l}\text { Potassium } \\
\text { (mg/L) }\end{array}$ & $\begin{array}{l}\text { Alkalinity, } \\
\text { fixed } \\
\text { endpoint } \\
\text { (mg/L) }\end{array}$ & $\begin{array}{c}\text { ANC } \\
\text { (mg/L) }\end{array}$ \\
\hline \multicolumn{14}{|c|}{ USGS-9S (measuring point altitude 582.05 feet above NGVD 29) } \\
\hline 06-03-99 & 1510 & 11.67 & 676 & 7.1 & 14.6 & 0.4 & -- & 98.7 & 21.9 & 8.54 & 2.38 & -- & 325 \\
\hline 09-01-99 & 1340 & -- & 658 & 7.2 & 14.1 & $>1.0$ & -- & 103 & 22.7 & 7.08 & 1.97 & 317 & 319 \\
\hline $03-03-00$ & 0810 & 24.89 & 665 & 7.1 & 14.7 & .8 & -- & 98.0 & 22.6 & 5.78 & 2.23 & 305 & 307 \\
\hline $08-22-00$ & 1620 & 23.49 & 652 & 7.0 & 15.2 & $>1.0$ & -- & 99.2 & 21.7 & 8.02 & 2.43 & 314 & 313 \\
\hline 02-20-01 & 1330 & 25.25 & 650 & 7.0 & 14.2 & .5 & -- & 109 & 22.7 & 7.14 & 2.20 & -- & 330 \\
\hline 08-23-01 & 1540 & 20.42 & 675 & 7.3 & 15.1 & $>1.0$ & -- & 105 & 22.8 & 7.40 & 2.06 & 307 & 262 \\
\hline 03-06-02 & 0900 & 26.09 & 787 & 7.0 & 14.7 & $>1.0$ & & 113 & 25.0 & 5.72 & 2.34 & 313 & 309 \\
\hline $08-21-02$ & 0950 & 24.49 & 744 & 7.2 & 15.6 & $>1.0$ & -- & 117 & 25.0 & 6.21 & 2.27 & 304 & 241 \\
\hline \multicolumn{14}{|c|}{ USGS-9D (measuring point altitude 582.16 feet above NGVD 29) } \\
\hline 06-03-99 & 1610 & 11.74 & 621 & 7.1 & 15.2 & $<.1$ & -- & 87.5 & 17.7 & 9.01 & 2.08 & -- & 278 \\
\hline$*$ & 1615 & 11.74 & 621 & 7.1 & 15.2 & $<.1$ & -- & 89.0 & 17.8 & 8.95 & 2.10 & -- & 278 \\
\hline 09-01-99 & 1250 & -- & 624 & 7.1 & 14.4 & $<.1$ & -- & 90.9 & 18.6 & 8.89 & 1.92 & 283 & 284 \\
\hline$*$ & 1255 & -- & 624 & 7.1 & 14.4 & $<.1$ & -- & 92.2 & 18.4 & 8.99 & 1.09 & 283 & 284 \\
\hline $03-03-00$ & 0850 & 24.95 & 592 & 7.2 & 14.3 & $<.1$ & -- & -- & -- & -- & -- & -- & -- \\
\hline * & 0855 & 24.95 & 592 & 7.2 & 14.3 & $<.1$ & -- & -- & -- & -- & -- & -- & -- \\
\hline $08-22-00$ & 1710 & 23.57 & 627 & 7.1 & 15.8 & $<.1$ & -- & 90.1 & 17.8 & 8.60 & 2.19 & 281 & 281 \\
\hline$*$ & 1715 & 23.57 & 627 & 7.1 & 15.8 & $<.1$ & -- & 91.4 & 18.0 & 8.62 & 2.17 & 280 & 275 \\
\hline 02-20-01 & 1450 & 25.33 & 573 & 7.0 & 13.9 & $<.1$ & -- & 89.0 & 17.2 & 8.98 & 1.85 & 272 & 272 \\
\hline$*$ & 1455 & 25.33 & 573 & 7.0 & 13.9 & $<.1$ & -- & 89.5 & 17.3 & 9.02 & 1.87 & 272 & 272 \\
\hline 08-23-01 & 1630 & 20.54 & 681 & 7.1 & 15.0 & $<.1$ & -- & 100 & 19.8 & 10 & 2.03 & 306 & 266 \\
\hline$*$ & 1635 & 20.54 & 681 & 7.1 & 15.0 & $<.1$ & -- & 100 & 19.9 & 9.88 & 2.20 & 260 & 261 \\
\hline 03-06-02 & 1000 & 26.19 & 635 & 7.1 & 14.0 & $<.1$ & -- & 93.2 & 18.2 & 9.48 & 2.10 & 290 & 291 \\
\hline$*$ & 1005 & 26.19 & 635 & 7.1 & 14.0 & $<.1$ & -- & -- & -- & -- & -- & -- & -- \\
\hline $08-21-02$ & 0850 & 24.58 & 670 & 7.1 & 15.6 & $<.1$ & -- & 103 & 19.5 & 9.93 & 2.28 & 301 & 259 \\
\hline$*$ & 0855 & 24.58 & 670 & 7.1 & 15.6 & $<.1$ & -- & 103 & 19.7 & 10 & 2.12 & 304 & 247 \\
\hline 04-01-03 & 1100 & 28.30 & 608 & 7.0 & 14.5 & $<.1$ & -- & -- & -- & -- & -- & -- & -- \\
\hline$*$ & 1105 & 28.30 & 608 & 7.0 & 14.5 & $<.1$ & -- & -- & -- & -- & -- & -- & -- \\
\hline
\end{tabular}


$\left[\mu \mathrm{S} / \mathrm{cm}\right.$, microsiemens per centimeter at 25 degrees Celsius; $\mathrm{pH}$, in standard units; ${ }^{\circ} \mathrm{C}$, degrees Celsius; constituent concentrations are dissolved, unless otherwise noted; mg/L, milligrams per liter; $\mathrm{CaCO}_{3}$, calcium carbonate; ANC, total acid neutralizing capacity, incremental titration; N, nitrogen; $\mu \mathrm{g} / \mathrm{L}$, micrograms per liter; NGVD 29, National Geodetic Vertical Datum of 1929; --, no data; $<$, less than; E, estimated; *, replicate sample; >, greater than]

\begin{tabular}{|c|c|c|c|c|c|c|c|c|c|c|c|c|c|}
\hline Date & Time & $\begin{array}{l}\text { Sulfate } \\
\text { (mg/L) }\end{array}$ & $\begin{array}{c}\text { Chloride } \\
\text { (mg/L) }\end{array}$ & $\begin{array}{c}\text { Fluoride } \\
\text { (mg/L) }\end{array}$ & $\begin{array}{c}\text { Residue, sum } \\
\text { of } \\
\text { constituents } \\
(\mathrm{mg} / \mathrm{L}))\end{array}$ & $\begin{array}{c}\text { Nitrite as N } \\
(\mathrm{mg} / \mathrm{L})\end{array}$ & $\begin{array}{c}\text { Nitrite plus } \\
\text { nitrate as N } \\
\text { (mg/L) }\end{array}$ & $\begin{array}{c}\text { Ammonia as } \\
\mathbf{N} \\
(\mathbf{m g} / \mathrm{L})\end{array}$ & $\begin{array}{l}\text { Ammonia } \\
\text { plus organic } \\
\text { N, total } \\
\text { (mg/L) }\end{array}$ & $\begin{array}{c}\text { Phosphorous, } \\
\text { total } \\
\text { (mg/L) }\end{array}$ & $\begin{array}{l}\text { Phosphorous, } \\
\text { dissolved } \\
\text { (mg/L) }\end{array}$ & $\begin{array}{c}\text { Ortho- } \\
\text { phosphorous } \\
\text { (mg/L) }\end{array}$ & $\begin{array}{l}\text { Iron } \\
(\mu \mathrm{g} / \mathrm{L})\end{array}$ \\
\hline \multicolumn{14}{|c|}{ USGS-9S } \\
\hline 06-03-99 & 1510 & 46.6 & 4.37 & 0.1 & -- & $<0.010$ & 0.24 & 0.04 & E0.10 & 0.10 & 0.10 & 0.08 & E10 \\
\hline 09-01-99 & 1340 & 50 & 4.90 & .1 & -- & $<.010$ & 1.00 & $<.02$ & $<.10$ & .12 & .13 & .12 & $<10$ \\
\hline $03-03-00$ & 0810 & 58.9 & 7.70 & .2 & -- & $<.010$ & 1.62 & $<.02$ & E.07 & .18 & .18 & .17 & $<10$ \\
\hline $08-22-00$ & 1620 & 56.8 & 4.10 & .1 & -- & .011 & .21 & $<.02$ & E.05 & .11 & .10 & .09 & $<10$ \\
\hline 02-20-01 & 1330 & 55.1 & 3.25 & .2 & -- & .008 & .24 & $<.04$ & $<.08$ & .12 & .10 & .09 & $<10$ \\
\hline 08-23-01 & 1540 & 48.2 & 4.96 & .2 & -- & .008 & .89 & 0.05 & .11 & .07 & .08 & E.10 & $<10$ \\
\hline 03-06-02 & 0900 & 55.4 & 49.0 & .2 & -- & E.004 & 1.04 & E.03 & $<.10$ & .07 & .08 & .07 & $<10$ \\
\hline 08-21-02 & 0950 & 48.4 & 36.5 & .1 & -- & E.005 & 1.19 & $<.04$ & E.05 & .07 & .07 & .07 & $<10$ \\
\hline \multicolumn{14}{|c|}{ USGS-9D } \\
\hline 06-03-99 & 1610 & 39.9 & 10.1 & .2 & 412 & $<.010$ & $<.05$ & .11 & .14 & .24 & .21 & .03 & 9,550 \\
\hline * & 1615 & 40.2 & 10.9 & .2 & 409 & .028 & $<.05$ & .15 & .14 & .26 & .28 & .20 & 9,580 \\
\hline 09-01-99 & 1250 & 38.0 & 10.9 & .2 & -- & .028 & $<.05$ & .08 & E.09 & .22 & .20 & .25 & 9,430 \\
\hline$*$ & 1255 & 38.1 & 10.2 & .2 & -- & .025 & $<.05$ & .07 & E.09 & .21 & .19 & .24 & 9,580 \\
\hline 03-03-00 & 0850 & -- & -- & -- & -- & .012 & $<.05$ & .07 & .12 & .24 & .06 & .14 & -- \\
\hline * & 0855 & -- & -- & -- & -- & .017 & $<.05$ & .07 & .12 & .23 & $<.05$ & .11 & -- \\
\hline $08-22-00$ & 1710 & 35.4 & 9.21 & .2 & -- & $<.010$ & $<.05$ & .06 & .13 & .25 & .22 & $<.01$ & 9,760 \\
\hline * & 1715 & 39.4 & 9.16 & .2 & -- & .022 & $<.05$ & .07 & .12 & .26 & .26 & .09 & 9,840 \\
\hline 02-20-01 & 1450 & 37.2 & 8.41 & .2 & -- & E.004 & $<.05$ & .06 & .09 & .27 & .21 & .09 & 9,550 \\
\hline * & 1455 & 37.0 & 8.63 & .2 & -- & E.003 & $<.05$ & .06 & .10 & .26 & .26 & .04 & 9,600 \\
\hline 08-23-01 & 1630 & 43.0 & 10.5 & .2 & -- & .007 & $<.05$ & .07 & .12 & .20 & .16 & E.21 & 10,200 \\
\hline$*$ & 1635 & 42.5 & 10.1 & .2 & -- & $<.006$ & $<.05$ & .07 & .09 & .22 & E.23 & E.04 & 10,300 \\
\hline 03-06-02 & 1000 & 40.9 & 9.86 & .2 & -- & .034 & .07 & .28 & .11 & .25 & .21 & .09 & 9,710 \\
\hline$*$ & 1005 & -- & -- & -- & -- & .031 & .09 & .14 & .12 & .25 & .21 & .12 & -- \\
\hline $08-21-02$ & 0850 & 40.6 & 10.4 & .2 & -- & $<.008$ & $<.05$ & .07 & .11 & .24 & .24 & .04 & 10,200 \\
\hline$*$ & 0855 & 40.3 & 10.3 & .2 & -- & .029 & $<.05$ & .11 & .12 & .24 & .23 & .22 & 10,300 \\
\hline 04-01-03 & 1100 & -- & 7.78 & -- & -- & -- & -- & -- & -- & -- & -- & -- & -- \\
\hline * & 1105 & -- & 7.43 & -- & -- & -- & -- & -- & -- & -- & -- & -- & -- \\
\hline
\end{tabular}


Table 1. Values of physical properties and concentrations of inorganic constituents and nutrients in samples from wells and surface-water sites, 1999-2005.-Continued

$\left[\mu \mathrm{S} / \mathrm{cm}\right.$, microsiemens per centimeter at 25 degrees Celsius; $\mathrm{pH}$, in standard units; ${ }^{\circ} \mathrm{C}$, degrees Celsius; constituent concentrations are dissolved, unless otherwise noted; mg/L, milligrams per liter; $\mathrm{CaCO}_{3}$, calcium carbonate; ANC, total acid neutralizing capacity, incremental titration; N, nitrogen; $\mu \mathrm{g} / \mathrm{L}$, micrograms per liter; NGVD 29, National Geodetic Vertical Datum of 1929; --, no data; $<$, less than; E, estimated; *, replicate sample; >, greater than]

\begin{tabular}{|c|c|c|c|c|c|c|c|c|c|c|c|c|c|}
\hline Date & Time & $\begin{array}{c}\text { Waterlevel } \\
\text { (feet below } \\
\text { measuring } \\
\text { point) }\end{array}$ & $\begin{array}{c}\text { Specific } \\
\text { conductance } \\
(\mu \mathrm{S} / \mathrm{cm})\end{array}$ & $\mathrm{pH}$ & $\begin{array}{c}\text { Temperature } \\
\left({ }^{\circ} \mathrm{C}\right)\end{array}$ & $\begin{array}{c}\text { Dissolved } \\
\text { oxygen } \\
\text { (mg/L) }\end{array}$ & $\begin{array}{c}\text { Hardness } \\
\text { (mg/L as } \\
\mathrm{CaCO}_{3} \text { ) }\end{array}$ & $\begin{array}{c}\text { Calcium } \\
\text { (mg/L) }\end{array}$ & $\begin{array}{l}\text { Magnesium } \\
(\mathbf{m g} / \mathbf{L})\end{array}$ & $\begin{array}{l}\text { Sodium } \\
\text { (mg/L) }\end{array}$ & $\begin{array}{l}\text { Potassium } \\
\text { (mg/L) }\end{array}$ & $\begin{array}{c}\text { Alkalinity, } \\
\text { fixed } \\
\text { endpoint } \\
\text { (mg/L) }\end{array}$ & $\begin{array}{l}\text { ANC } \\
\text { (mg/L) }\end{array}$ \\
\hline \multicolumn{14}{|c|}{ City of Columbia public-supply well 5} \\
\hline $04-03-03$ & 0840 & -- & 979 & 6.8 & 16.6 & -- & -- & -- & -- & -- & -- & -- & -- \\
\hline $08-28-03$ & 1220 & -- & 848 & 7.0 & -- & -- & -- & -- & -- & -- & -- & -- & -- \\
\hline \multicolumn{14}{|c|}{ Blew hole } \\
\hline 08-26-99 & 1340 & -- & 881 & 7.7 & 26.8 & 6.7 & -- & 83.8 & 18.7 & 58.0 & 6.29 & 232 & 226 \\
\hline 02-29-00 & 1110 & -- & 1,030 & 6.8 & 10.8 & 11.1 & -- & 104 & 22.0 & 91.2 & 5.85 & 255 & 265 \\
\hline $08-30-00$ & 0800 & -- & 566 & 7.3 & 30.0 & 3.3 & -- & 52.3 & 12.8 & 46.2 & 6.03 & 134 & 134 \\
\hline $02-27-01$ & 1440 & -- & 234 & 7.2 & 5.3 & 10.2 & -- & 33.7 & 6.0 & 14.3 & 4.44 & 82 & 81 \\
\hline 08-29-01 & 0830 & -- & 1,020 & 7.4 & 26.6 & 3.8 & -- & 73.8 & 17.8 & 106 & 7.42 & 239 & 236 \\
\hline $03-05-02$ & 1330 & -- & 1,070 & 8.2 & 5.5 & 15.3 & -- & 74.1 & 19.5 & 97.4 & 6.00 & 231 & 234 \\
\hline \multicolumn{14}{|c|}{ City outflow } \\
\hline 08-31-99 & 1050 & -- & 1,540 & 7.5 & 21.1 & 3.5 & -- & 45.7 & 23.3 & 207 & 41.5 & 257 & 216 \\
\hline $03-02-00$ & 1140 & -- & 1,470 & 7.3 & 10.8 & 5.9 & -- & 53.2 & 20.0 & 198 & 36.6 & 241 & 211 \\
\hline $08-29-00$ & 1430 & -- & 1,400 & 7.4 & 28.8 & 2.7 & -- & 60.3 & 20.1 & 160 & 24.3 & 245 & 194 \\
\hline $02-28-01$ & 1440 & -- & 1,210 & 7.7 & 7.6 & 5.7 & -- & 60.2 & 15.8 & 125 & 23.1 & 241 & 172 \\
\hline 08-24-01 & 1030 & -- & 1,600 & 7.4 & 24.5 & 2.4 & -- & 43.9 & 21.4 & 196 & -- & 209 & 157 \\
\hline 03-05-02 & 1510 & -- & 1,590 & 8.0 & 11.5 & 12.0 & -- & 48.4 & 18.0 & 195 & 23.2 & 229 & 178 \\
\hline 04-02-03 & 1400 & -- & 1,500 & 7.8 & 21.8 & 8.8 & -- & -- & -- & -- & -- & -- & -- \\
\hline 04-09-03 & 1630 & -- & 1,440 & 7.6 & 15.7 & -- & -- & -- & -- & -- & -- & -- & -- \\
\hline $08-27-03$ & 1450 & -- & 1,780 & 7.3 & 27.3 & 2.5 & -- & -- & -- & -- & -- & -- & -- \\
\hline \multicolumn{14}{|c|}{ Eagle Bluffs outflow } \\
\hline 08-30-99 & 1540 & -- & 1,490 & 8.8 & 25.9 & 1.1 & -- & 48.6 & 23.6 & 208 & 38.2 & 244 & 230 \\
\hline $03-02-00$ & 1100 & -- & 1,220 & 8.8 & 10.0 & 7.8 & -- & 44.7 & 17.7 & 164 & 32.5 & 202 & 179 \\
\hline 08-29-00 & 1530 & -- & 1,400 & 7.5 & 31.7 & .7 & -- & 63.3 & 19.7 & 171 & 29.1 & 240 & 203 \\
\hline 02-28-01 & 1300 & -- & 1,180 & 8.2 & 4.1 & 13.4 & -- & 44.7 & 14.8 & 135 & 28.1 & 215 & 145 \\
\hline 08-24-01 & 0940 & -- & 1,600 & 9.0 & 27.0 & 2.7 & -- & 50.9 & 22.5 & 219 & 34.5 & 226 & 215 \\
\hline $03-05-02$ & 1430 & -- & 1,640 & 8.1 & 5.5 & 16.0 & -- & 47.0 & 21.0 & 205 & 34.9 & 246 & 220 \\
\hline
\end{tabular}


$\left[\mu \mathrm{S} / \mathrm{cm}\right.$, microsiemens per centimeter at 25 degrees Celsius; $\mathrm{pH}$, in standard units; ${ }^{\circ} \mathrm{C}$, degrees Celsius; constituent concentrations are dissolved, unless otherwise noted; mg/L, milligrams per liter; $\mathrm{CaCO}_{3}$, calcium carbonate; ANC, total acid neutralizing capacity, incremental titration; N, nitrogen; $\mu \mathrm{g} / \mathrm{L}$, micrograms per liter; NGVD 29, National Geodetic Vertical Datum of 1929; --, no data; $<$, less than; E, estimated; *, replicate sample; >, greater than]

\begin{tabular}{|c|c|c|c|c|c|c|c|c|c|c|c|c|c|}
\hline Date & Time & $\begin{array}{l}\text { Sulfate } \\
\text { (mg/L) }\end{array}$ & $\begin{array}{c}\text { Chloride } \\
\text { (mg/L) }\end{array}$ & $\begin{array}{c}\text { Fluoride } \\
\text { (mg/L) }\end{array}$ & $\begin{array}{l}\text { Residue, sum } \\
\text { of } \\
\text { constituents } \\
(\mathrm{mg} / \mathrm{L}))\end{array}$ & $\begin{array}{c}\text { Nitrite as N } \\
(\mathbf{m g} / \mathbf{L})\end{array}$ & $\begin{array}{c}\text { Nitrite plus } \\
\text { nitrate as N } \\
\text { (mg/L) }\end{array}$ & $\begin{array}{c}\text { Ammonia as } \\
\mathbf{N} \\
(\mathbf{m g} / \mathrm{L})\end{array}$ & $\begin{array}{c}\text { Ammonia } \\
\text { plus organic } \\
\text { N, total } \\
\text { (mg/L) }\end{array}$ & $\begin{array}{c}\text { Phosphorous, } \\
\text { total } \\
\text { (mg/L) }\end{array}$ & $\begin{array}{l}\text { Phosphorous, } \\
\text { dissolved } \\
\text { (mg/L) }\end{array}$ & $\begin{array}{c}\text { Ortho- } \\
\text { phosphorous } \\
\text { (mg/L) }\end{array}$ & $\begin{array}{l}\text { Iron } \\
(\mu \mathrm{g} / \mathrm{L})\end{array}$ \\
\hline \multicolumn{14}{|c|}{ City of Columbia public-supply well 5} \\
\hline 04-03-03 & 0840 & -- & 64.5 & -- & -- & -- & -- & -- & -- & -- & -- & -- & -- \\
\hline 08-28-03 & 1220 & -- & 50.3 & -- & -- & -- & -- & -- & -- & -- & -- & -- & -- \\
\hline \multicolumn{14}{|c|}{ Blew hole } \\
\hline 08-26-99 & 1340 & 81.9 & 105 & 0.3 & -- & $<0.010$ & $<0.05$ & 0.07 & 1.0 & 0.18 & E.04 & $<0.01$ & 10 \\
\hline 02-29-00 & 1110 & 82.7 & 170 & .2 & -- & $<.010$ & $<.05$ & $<.02$ & 1.0 & .17 & $<.05$ & $<.01$ & 13 \\
\hline $08-30-00$ & 0800 & 42.8 & 78.6 & .3 & -- & $<.010$ & $<.05$ & .06 & 1.4 & .23 & E.05 & $<.01$ & 28 \\
\hline $02-27-01$ & 1440 & 27.6 & 23.6 & E. 1 & -- & .013 & .59 & .09 & 1.4 & .29 & .07 & .06 & 41 \\
\hline 08-29-01 & 830 & 67.4 & 131 & .3 & -- & $<.006$ & $<.05$ & $<.04$ & 1.1 & .17 & E.03 & E.01 & 30 \\
\hline $03-05-02$ & 1330 & 84.6 & 137 & .3 & -- & $<.008$ & $<.05$ & $<.04$ & .88 & .09 & $<.06$ & $<.02$ & E6 \\
\hline \multicolumn{14}{|c|}{ City outflow } \\
\hline 08-31-99 & 1050 & 98.3 & 280 & 1.1 & -- & .017 & $<.05$ & 5.91 & 5.2 & 2.83 & 2.88 & 2.86 & 126 \\
\hline $03-02-00$ & 1140 & 108 & 280 & 1.0 & -- & .243 & .93 & 4.85 & 5.4 & 2.13 & 1.95 & 1.67 & 89 \\
\hline $08-29-00$ & 1430 & 107 & 211 & 1.1 & -- & $<.010$ & $<.05$ & 7.94 & 8.7 & 1.81 & 1.78 & 1.50 & 174 \\
\hline $02-28-01$ & 1440 & 93.8 & 188 & .8 & -- & .038 & .12 & 9.63 & 13 & 1.81 & 1.42 & 1.26 & 140 \\
\hline 08-24-01 & 1030 & 101 & 241 & .9 & -- & .008 & E.03 & E14.6 & 18 & 3.06 & 2.86 & E2.40 & 177 \\
\hline $03-05-02$ & 1510 & 91.5 & 278 & .9 & -- & .059 & .28 & 14.3 & 15 & 2.55 & 2.17 & 2.34 & 64 \\
\hline 04-02-03 & 1400 & -- & 285 & -- & -- & -- & -- & -- & -- & -- & -- & -- & -- \\
\hline 04-09-03 & 1630 & -- & -- & -- & -- & -- & -- & -- & -- & -- & -- & -- & -- \\
\hline $08-27-03$ & 1450 & -- & 289 & -- & -- & -- & -- & -- & -- & -- & -- & -- & -- \\
\hline \multicolumn{14}{|c|}{ Eagle Bluffs outflow } \\
\hline 08-30-99 & 1540 & 108 & 271 & 1.2 & -- & .661 & 1.16 & 1.33 & 5.2 & 1.75 & 1.26 & 1.10 & E8 \\
\hline $03-02-00$ & 1100 & 91.0 & 218 & .9 & -- & .230 & 1.24 & 3.35 & 5.8 & 2.06 & 1.67 & 1.43 & 32 \\
\hline 08-29-00 & 1530 & 113 & 223 & 1.1 & -- & .153 & .29 & 5.78 & 6.8 & 2.04 & 1.93 & 1.78 & 20 \\
\hline $02-28-01$ & 1300 & 80.8 & 203 & .8 & -- & .086 & 1.18 & 9.93 & 14 & 2.38 & 1.94 & 1.63 & 79 \\
\hline 08-24-01 & 0940 & 119 & 278 & 1.0 & -- & E. 144 & E.18 & E.55 & 3.4 & .86 & .46 & E. 34 & E10 \\
\hline $03-05-02$ & 1430 & 102 & 282 & 1.0 & -- & .116 & 1.53 & 7.31 & 9.4 & 2.02 & 1.89 & 1.91 & 31 \\
\hline
\end{tabular}


Table 1. Values of physical properties and concentrations of inorganic constituents and nutrients in samples from wells and surface-water sites, 1999-2005._Continued

$\left[\mu \mathrm{S} / \mathrm{cm}\right.$, microsiemens per centimeter at 25 degrees Celsius; $\mathrm{pH}$, in standard units; ${ }^{\circ} \mathrm{C}$, degrees Celsius; constituent concentrations are dissolved, unless otherwise noted; mg/L, milligrams per liter; $\mathrm{CaCO}_{3}$, calcium carbonate; ANC, total acid neutralizing capacity, incremental titration; N, nitrogen; $\mu \mathrm{g} / \mathrm{L}$, micrograms per liter; NGVD 29, National Geodetic Vertical Datum of 1929; --, no data; $<$, less than; E, estimated; *, replicate sample; >, greater than]

\begin{tabular}{|c|c|c|c|c|c|c|c|c|c|c|c|c|c|}
\hline Date & Time & $\begin{array}{c}\text { Waterlevel } \\
\text { (feet below } \\
\text { measuring } \\
\text { point) }\end{array}$ & $\begin{array}{c}\text { Specific } \\
\text { conductance } \\
(\mu \mathrm{S} / \mathrm{cm})\end{array}$ & $\mathrm{pH}$ & $\begin{array}{c}\text { Temperature } \\
\left({ }^{\circ} \mathrm{C}\right)\end{array}$ & $\begin{array}{c}\text { Dissolved } \\
\text { oxygen } \\
\text { (mg/L) }\end{array}$ & $\begin{array}{c}\text { Hardness } \\
\text { (mg/L as } \\
\mathrm{CaCO}_{3} \text { ) }\end{array}$ & $\begin{array}{c}\text { Calcium } \\
\text { (mg/L) }\end{array}$ & $\begin{array}{l}\text { Magnesium } \\
(\mathbf{m g} / \mathbf{L})\end{array}$ & $\begin{array}{c}\text { Sodium } \\
\text { (mg/L) }\end{array}$ & $\begin{array}{l}\text { Potassium } \\
\text { (mg/L) }\end{array}$ & $\begin{array}{c}\text { Alkalinity, } \\
\text { fixed } \\
\text { endpoint } \\
\text { (mg/L) }\end{array}$ & $\begin{array}{l}\text { ANC } \\
\text { (mg/L) }\end{array}$ \\
\hline \multicolumn{14}{|c|}{ Perche Creek } \\
\hline $08-26-99$ & 830 & -- & 653 & 7.3 & 24.6 & 4.8 & -- & 91.6 & 16.1 & 17.7 & 3.91 & 292 & 281 \\
\hline $03-02-00$ & 1410 & -- & 491 & 7.0 & 10.9 & 6.9 & -- & 64.2 & 12.8 & 18.2 & 5.24 & 131 & 131 \\
\hline $08-30-00$ & 0930 & -- & 378 & 7.4 & 28.9 & 5.3 & -- & 57.2 & 9.49 & 11.4 & 4.86 & 138 & 137 \\
\hline $02-27-01$ & 1450 & -- & 234 & 7.4 & 4.8 & 10.8 & -- & 31.6 & 5.24 & 6.18 & 4.09 & 71 & 71 \\
\hline 08-29-01 & 1000 & -- & 304 & 7.0 & 25.3 & 4.1 & -- & 40.9 & 6.19 & 8.64 & 5.37 & 101 & 99 \\
\hline $03-05-02$ & 1200 & -- & 700 & 7.2 & 2.4 & 14.0 & -- & 79.9 & 13.4 & 39.9 & 4.32 & 170 & 168 \\
\hline \multicolumn{14}{|c|}{ Missouri River } \\
\hline $04-02-03$ & 1040 & -- & 617 & 8.0 & 12.5 & 11.2 & -- & -- & -- & -- & -- & -- & -- \\
\hline $08-27-03$ & 1540 & -- & 798 & 8.1 & 30.1 & 5.9 & -- & -- & -- & -- & -- & -- & -- \\
\hline
\end{tabular}


$\left[\mu \mathrm{S} / \mathrm{cm}\right.$, microsiemens per centimeter at 25 degrees Celsius; $\mathrm{pH}$, in standard units; ${ }^{\circ} \mathrm{C}$, degrees Celsius; constituent concentrations are dissolved, unless otherwise noted; mg/L, milligrams per liter; $\mathrm{CaCO}_{3}$, calcium carbonate; ANC, total acid neutralizing capacity, incremental titration; N, nitrogen; $\mu \mathrm{g} / \mathrm{L}$, micrograms per liter; NGVD 29, National Geodetic Vertical Datum of 1929; --, no data; $<$, less than; E, estimated; *, replicate sample; >, greater than]

\begin{tabular}{|c|c|c|c|c|c|c|c|c|c|c|c|c|c|}
\hline Date & Time & $\begin{array}{l}\text { Sulfate } \\
\text { (mg/L) }\end{array}$ & $\begin{array}{c}\text { Chloride } \\
\text { (mg/L) }\end{array}$ & $\begin{array}{c}\text { Fluoride } \\
\text { (mg/L) }\end{array}$ & $\begin{array}{l}\text { Residue, sum } \\
\text { of } \\
\text { constituents } \\
(\mathrm{mg} / \mathrm{L}))\end{array}$ & $\begin{array}{l}\text { Nitrite as N } \\
(\mathrm{mg} / \mathrm{L})\end{array}$ & $\begin{array}{c}\text { Nitrite plus } \\
\text { nitrate as N } \\
(\mathrm{mg} / \mathrm{L})\end{array}$ & $\begin{array}{c}\text { Ammonia as } \\
\mathbf{N} \\
(\mathbf{m g} / \mathbf{L})\end{array}$ & $\begin{array}{c}\text { Ammonia } \\
\text { plus organic } \\
\text { N, total } \\
\text { (mg/L) }\end{array}$ & $\begin{array}{c}\text { Phosphorous, } \\
\text { total } \\
\text { (mg/L) }\end{array}$ & $\begin{array}{l}\text { Phosphorous, } \\
\text { dissolved } \\
\text { (mg/L) }\end{array}$ & $\begin{array}{c}\text { Ortho- } \\
\text { phosphorous } \\
\text { (mg/L) }\end{array}$ & $\begin{array}{l}\text { Iron } \\
(\mu \mathrm{g} / \mathrm{L})\end{array}$ \\
\hline \multicolumn{14}{|c|}{ Perche Creek } \\
\hline 08-26-99 & 0830 & 39.0 & 24.3 & 0.2 & -- & $<0.010$ & $<0.05$ & 0.14 & 0.94 & 0.08 & $<0.05$ & $<0.01$ & $<10$ \\
\hline $03-02-00$ & 1410 & 96.5 & 27.1 & .2 & -- & .015 & .51 & .25 & 1.2 & .27 & $<.05$ & $<.01$ & 26 \\
\hline 08-30-00 & 0930 & 50.9 & 12.1 & .2 & -- & .012 & .17 & $<.02$ & .74 & .14 & E.03 & .02 & $<10$ \\
\hline 02-27-01 & 1450 & 33.4 & 8.67 & E. 1 & -- & .011 & .75 & .07 & 1.3 & .31 & E.06 & .06 & 25 \\
\hline 08-29-01 & 1000 & 35.6 & 13.1 & .2 & -- & E.029 & E.36 & E.07 & .72 & .14 & $<.06$ & E.01 & E6 \\
\hline 03-05-02 & 1200 & 91.9 & 63.6 & .2 & -- & E.006 & .30 & .12 & .45 & .07 & $<.06$ & $<.02$ & 134 \\
\hline \multicolumn{14}{|c|}{ Missouri River } \\
\hline 04-02-03 & 1040 & -- & 25.8 & -- & -- & -- & -- & -- & -- & -- & -- & -- & -- \\
\hline $08-27-03$ & 1540 & -- & 26.2 & -- & -- & -- & -- & -- & -- & -- & -- & -- & -- \\
\hline
\end{tabular}


Table 2. Concentrations of trace elements, organic carbon, bromide, and silica in samples from selected monitoring wells, 1999.

[ $\mu \mathrm{g} / \mathrm{L}$, micrograms per liter; constituent concentrations are dissolved, unless otherwise noted; <, less than; --, no data; E, estimated; mg/L, milligrams per liter; COD, chemical oxygen demand; *, replicate sample]

\begin{tabular}{|c|c|c|c|c|c|c|c|c|c|c|c|c|c|c|}
\hline Date & Time & $\underset{(\mu \mathrm{g} / \mathrm{L})}{\text { Aluminum }}$ & $\begin{array}{c}\text { Arsenic } \\
(\mu \mathrm{g} / \mathrm{L})\end{array}$ & $\begin{array}{l}\text { Barium } \\
(\mu \mathrm{g} / \mathrm{L})\end{array}$ & $\begin{array}{c}\text { Beryllium } \\
(\mu \mathrm{g} / \mathrm{L})\end{array}$ & $\begin{array}{l}\text { Boron } \\
(\mu \mathrm{g} / \mathrm{L})\end{array}$ & $\begin{array}{c}\text { Cadmium } \\
(\mu \mathrm{g} / \mathrm{L})\end{array}$ & $\begin{array}{c}\text { Chromium } \\
(\mu \mathrm{g} / \mathrm{L})\end{array}$ & $\begin{array}{l}\text { Cobalt } \\
(\mu \mathrm{g} / \mathrm{L})\end{array}$ & $\begin{array}{l}\text { Copper } \\
(\mu \mathrm{g} / \mathrm{L})\end{array}$ & $\begin{array}{l}\text { Lead } \\
(\mu \mathrm{g} / \mathrm{L})\end{array}$ & $\begin{array}{l}\text { Lithium } \\
(\mu \mathrm{g} / \mathrm{L})\end{array}$ & $\begin{array}{c}\text { Manganese } \\
(\mu \mathrm{g} / \mathrm{L})\end{array}$ & $\begin{array}{c}\text { Mercury } \\
(\mu \mathrm{g} / \mathrm{L})\end{array}$ \\
\hline \multicolumn{15}{|c|}{ MW1-1A } \\
\hline 06-03-99 & 1040 & $<10$ & 6 & 42.4 & $<1.6$ & 55 & $<8$ & $<14$ & -- & $<10$ & $<1$ & 20 & 3,280 & $<0.1$ \\
\hline 09-02-99 & 1000 & $<20$ & 6 & 46.2 & $<1.6$ & 46 & $<8$ & $<14$ & -- & $<10$ & $<1$ & 26 & 3,660 & $<.1$ \\
\hline \multicolumn{15}{|c|}{ MW1-1B } \\
\hline 06-03-99 & 1000 & $<10$ & 1 & 417 & $<1.6$ & 90 & $<8$ & $<14$ & -- & $<10$ & $<1$ & 20 & 2,250 & $<.1$ \\
\hline 09-02-99 & 1050 & $<20$ & 2 & 418 & $<1.6$ & 72 & $<8$ & $<14$ & -- & $<10$ & $<1$ & 24 & 2,340 & $<.1$ \\
\hline \multicolumn{15}{|c|}{ MW1-2A } \\
\hline 06-03-99 & 0900 & $<10$ & 2 & 432 & $<1.6$ & 190 & $<8$ & $<14$ & $<7$ & $<10$ & $<1$ & 13 & 411 & .1 \\
\hline 09-02-99 & 0810 & $<20$ & 2 & 486 & $<1.6$ & 218 & $<8$ & $<14$ & E7 & $<10$ & $<1$ & 17 & 432 & $<.1$ \\
\hline \multicolumn{15}{|c|}{ MW1-2B } \\
\hline 06-03-99 & 0820 & $<10$ & $<1$ & 1,330 & $<1.6$ & 83 & $<8$ & $<14$ & -- & $<10$ & $<1$ & 25 & 422 & $<.1$ \\
\hline 09-02-99 & 0900 & $<20$ & $<1$ & 1,360 & $<1.6$ & 87 & $<8$ & $<14$ & -- & $<10$ & $<1$ & 27 & 408 & $<.1$ \\
\hline \multicolumn{15}{|c|}{ MW1-3A } \\
\hline 06-03-99 & 1250 & $<10$ & 14 & 418 & $<1.6$ & 59 & $<8$ & $<14$ & -- & $<10$ & $<1$ & 19 & 431 & $<.1$ \\
\hline 09-01-99 & 1440 & $<20$ & 11 & 512 & $<1.6$ & 46 & $<8$ & $<14$ & -- & $<10$ & $<1$ & 23 & 668 & $<.1$ \\
\hline 06-03-99 & 1350 & $<10$ & $<1$ & 773 & $<1.6$ & 53 & $<8$ & $<14$ & -- & $<10$ & $<1$ & 15 & 294 & $<.1$ \\
\hline 09-01-99 & 1550 & $<20$ & $<1$ & 881 & $<1.6$ & 46 & $<8$ & $<14$ & -- & $<10$ & $<1$ & 19 & 347 & $<.1$ \\
\hline \multicolumn{15}{|c|}{ MW1-4A } \\
\hline 06-01-99 & 1530 & $<10$ & $<1$ & 176 & $<1.6$ & 53 & $<8$ & $<14$ & $<7$ & $<10$ & $<1$ & 45 & 508 & $<.1$ \\
\hline$*$ & 1535 & $<10$ & $<1$ & 180 & $<1.6$ & 49 & $<8$ & $<14$ & $<7$ & $<10$ & $<1$ & 47 & 540 & $<.1$ \\
\hline 09-02-99 & 1410 & $<20$ & 1 & 187 & $<1.6$ & 55 & $<8$ & $<14$ & $<13$ & $<10$ & $<1$ & 53 & 581 & $<.1$ \\
\hline$*$ & 1415 & $<20$ & 1 & 189 & $<1.6$ & 52 & $<8$ & $<14$ & $<13$ & $<10$ & $<1$ & 53 & 601 & -- \\
\hline \multicolumn{15}{|c|}{ MW1-4B } \\
\hline 06-01-99 & 1430 & $<10$ & 17 & 239 & $<1.6$ & 89 & $<8$ & $<14$ & 8 & $<10$ & $<1$ & 28 & 155 & $<.1$ \\
\hline 09-02-99 & 1320 & $<20$ & 37 & 264 & $<1.6$ & 116 & $<8$ & $<14$ & -- & $<10$ & $<1$ & 23 & 265 & $<.1$ \\
\hline \multicolumn{15}{|c|}{ MW2-1A } \\
\hline 06-02-99 & 1300 & $<10$ & $<1$ & 539 & $<1.6$ & 66 & $<8$ & $<14$ & $<7$ & $<10$ & $<1$ & 30 & 72.6 & $<.1$ \\
\hline 08-27-99 & 0810 & $<20$ & 2 & 508 & $<1.6$ & 63 & $<8$ & $<14$ & 8 & $<10$ & $<1$ & 3 & 29.4 & $<.1$ \\
\hline
\end{tabular}


Table 2. Concentrations of trace elements, organic carbon, bromide, and silica in samples from selected monitoring wells, 1999.-Continued

$[\mu \mathrm{g} / \mathrm{L}$, micrograms per liter; constituent concentrations are dissolved, unless otherwise noted; <, less than; --, no data; E, estimated; mg/L, milligrams per liter; COD, chemical oxygen demand; *, replicate sample]

\begin{tabular}{|c|c|c|c|c|c|c|c|c|c|c|c|c|c|}
\hline Date & Time & $\begin{array}{l}\text { Molybdenum } \\
(\mu \mathrm{g} / \mathrm{L})\end{array}$ & $\begin{array}{l}\text { Nickel } \\
(\mu \mathrm{g} / \mathrm{L})\end{array}$ & $\begin{array}{c}\text { Selenium } \\
(\mu \mathrm{g} / \mathrm{L})\end{array}$ & $\begin{array}{l}\text { Silver } \\
(\mu \mathrm{g} / \mathrm{L})\end{array}$ & $\begin{array}{c}\text { Strontium } \\
(\mu \mathrm{g} / \mathrm{L})\end{array}$ & $\begin{array}{c}\text { Vanadium } \\
(\mu \mathrm{g} / \mathrm{L})\end{array}$ & $\begin{array}{l}\text { Zinc } \\
(\mu \mathrm{g} / \mathrm{L})\end{array}$ & $\begin{array}{c}\text { Organic } \\
\text { carbon, } \\
\text { (mg/L) }\end{array}$ & $\begin{array}{c}\text { Organic } \\
\text { carbon, } \\
\text { total } \\
(\mathrm{mg} / \mathrm{L})\end{array}$ & $\begin{array}{c}\text { COD, } \\
\text { high level } \\
\text { (mg/L) }\end{array}$ & $\begin{array}{c}\text { Bromide } \\
\text { (mg/L) }\end{array}$ & $\begin{array}{l}\text { Silica } \\
\text { (mg/L) }\end{array}$ \\
\hline \multicolumn{14}{|c|}{ MW1-1A } \\
\hline 06-03-99 & 1040 & $<50$ & $<40$ & $<1$ & $<4$ & 449 & $<10$ & $<20$ & 2.6 & 3.3 & 30 & 0.06 & 37.9 \\
\hline 09-02-99 & 1000 & $<30$ & $<40$ & 1 & $<7$ & 494 & $<10$ & $<20$ & 3.5 & 4.2 & $<10$ & .07 & 39.9 \\
\hline \multicolumn{14}{|c|}{ MW1-1B } \\
\hline 06-03-99 & 1000 & $<50$ & $<40$ & $<1$ & $<4$ & 931 & $<10$ & $<20$ & 2.2 & 3.1 & 20 & .09 & 45.0 \\
\hline 09-02-99 & 1050 & $<30$ & $<40$ & $<1$ & $<7$ & 926 & $<10$ & $<20$ & 3.5 & 7.4 & $<10$ & .08 & 47.3 \\
\hline \multicolumn{14}{|c|}{ MW1-2A } \\
\hline 06-03-99 & 0900 & $<50$ & $<40$ & $<1$ & $<4$ & 177 & $<10$ & $<20$ & 2.7 & 3.6 & 30 & .33 & 23.1 \\
\hline 09-02-99 & 0810 & $<30$ & $<40$ & $<1$ & $<7$ & 190 & $<10$ & $<20$ & 3.2 & 3.9 & $<10$ & .26 & 24.3 \\
\hline \multicolumn{14}{|c|}{ MW1-2B } \\
\hline 06-03-99 & 0820 & $<50$ & $<40$ & $<1$ & $<4$ & 345 & $<10$ & $<20$ & 2.4 & 2.4 & 30 & .28 & 32.6 \\
\hline 09-02-99 & 0900 & $<30$ & $<40$ & $<1$ & $<7$ & 338 & $<10$ & $<20$ & 3.2 & 4.2 & $<10$ & .49 & 33.7 \\
\hline \multicolumn{14}{|c|}{ MW1-3A } \\
\hline 06-03-99 & 1250 & $<50$ & $<40$ & $<1$ & $<4$ & 477 & $<10$ & $<20$ & 1.3 & 2.8 & 20 & .04 & 43.4 \\
\hline 09-01-99 & 1440 & $<30$ & $<40$ & $<1$ & $<7$ & 492 & $<10$ & $<20$ & 1.8 & 2.3 & $<10$ & .07 & 39.9 \\
\hline \multicolumn{14}{|c|}{ MW1-3B } \\
\hline 06-03-99 & 1350 & $<50$ & $<40$ & $<1$ & $<4$ & 349 & $<10$ & $<20$ & 1.4 & 1.3 & 20 & .09 & 33.8 \\
\hline 09-01-99 & 1550 & $<30$ & $<40$ & $<1$ & $<7$ & 386 & $<10$ & $<20$ & 1.8 & 2.1 & $<10$ & .11 & 33.5 \\
\hline \multicolumn{14}{|c|}{ MW1-4A } \\
\hline 06-01-99 & 1530 & $<50$ & $<40$ & $<1$ & $<4$ & 565 & $<10$ & $<20$ & 1.4 & 1.7 & 20 & .07 & 16.0 \\
\hline * & 1535 & $<50$ & $<40$ & $<1$ & $<4$ & 590 & $<10$ & $<20$ & 1.3 & 1.2 & 20 & .07 & 16.7 \\
\hline 09-02-99 & 1410 & $<30$ & $<40$ & 1 & $<7$ & 607 & $<10$ & $<20$ & 1.9 & 2.0 & $<10$ & .08 & 17.9 \\
\hline$*$ & 1415 & $<30$ & $<40$ & $<1$ & $<7$ & 612 & $<10$ & $<20$ & 2.0 & 2.0 & $<10$ & .08 & 18.0 \\
\hline \multicolumn{14}{|c|}{ MW1-4B } \\
\hline 06-01-99 & 1430 & $<50$ & $<40$ & 1 & $<4$ & 485 & $<10$ & E8 & 2.0 & 3.3 & 20 & .04 & 28.2 \\
\hline 09-02-99 & 1320 & $<30$ & $<40$ & $<1$ & $<7$ & 437 & E5 & $<20$ & 3.2 & 4.4 & $<10$ & $<.01$ & 34.2 \\
\hline \multicolumn{14}{|c|}{ MW2-1A } \\
\hline 06-02-99 & 1300 & $<50$ & $<40$ & $<1$ & $<4$ & 512 & $<10$ & $<20$ & 1.5 & 2.8 & 10 & .03 & 25.8 \\
\hline 08-27-99 & 0810 & $<30$ & $<40$ & $<1$ & $<7$ & 418 & $<10$ & $<20$ & -- & -- & -- & .03 & 28.2 \\
\hline
\end{tabular}


Table 2. Concentrations of trace elements, organic carbon, bromide, and silica in samples from selected monitoring wells, 1999.-Continued

[ $\mu \mathrm{g} / \mathrm{L}$, micrograms per liter; constituent concentrations are dissolved, unless otherwise noted; <, less than; --, no data; E, estimated; mg/L, milligrams per liter; COD, chemical oxygen demand; *, replicate sample]

\begin{tabular}{|c|c|c|c|c|c|c|c|c|c|c|c|c|c|c|}
\hline Date & Time & $\begin{array}{c}\text { Aluminum } \\
(\mu \mathrm{g} / \mathrm{L})\end{array}$ & $\begin{array}{c}\text { Arsenic } \\
(\mu \mathrm{g} / \mathrm{L})\end{array}$ & $\begin{array}{l}\text { Barium } \\
(\mu \mathrm{g} / \mathrm{L})\end{array}$ & $\begin{array}{c}\text { Beryllium } \\
(\mu \mathrm{g} / \mathrm{L})\end{array}$ & $\begin{array}{l}\text { Boron } \\
(\mu \mathrm{g} / \mathrm{L})\end{array}$ & $\begin{array}{c}\text { Cadmium } \\
(\mu \mathrm{g} / \mathrm{L})\end{array}$ & $\begin{array}{c}\text { Chromium } \\
(\mu \mathrm{g} / \mathrm{L})\end{array}$ & $\begin{array}{l}\text { Cobalt } \\
(\mu \mathrm{g} / \mathrm{L})\end{array}$ & $\begin{array}{l}\text { Copper } \\
(\mu \mathrm{g} / \mathrm{L})\end{array}$ & $\begin{array}{l}\text { Lead } \\
(\mu \mathrm{g} / \mathrm{L})\end{array}$ & $\begin{array}{l}\text { Lithium } \\
(\mu \mathrm{g} / \mathrm{L})\end{array}$ & $\begin{array}{c}\text { Manganese } \\
(\mu \mathrm{g} / \mathrm{L})\end{array}$ & $\begin{array}{c}\text { Mercury } \\
(\mu \mathrm{g} / \mathrm{L})\end{array}$ \\
\hline \multicolumn{15}{|c|}{ MW2-1B } \\
\hline 06-02-99 & 1400 & $<10$ & $<1$ & 1,330 & $<1.6$ & 85 & $<8$ & $<14$ & -- & $<10$ & $<1$ & 40 & 321 & $<0.1$ \\
\hline 08-27-99 & 0900 & $<20$ & $<1$ & 1,340 & $<1.6$ & 85 & $<8$ & $<14$ & -- & $<10$ & $<1$ & 25 & 343 & $<.1$ \\
\hline \multicolumn{15}{|c|}{ MW3-1A } \\
\hline 06-02-99 & 1440 & $<10$ & $<1$ & 226 & $<1.6$ & 55 & $<8$ & $<14$ & $<7$ & $<10$ & $<1$ & 26 & 278 & $<.1$ \\
\hline 09-01-99 & 0900 & $<20$ & 2 & 234 & $<1.6$ & 57 & $<8$ & $<14$ & $<13$ & $<10$ & $<1$ & 28 & 291 & $<.1$ \\
\hline \multicolumn{15}{|c|}{ MW3-1B } \\
\hline 06-02-99 & 1530 & $<10$ & $<1$ & 731 & $<1.6$ & 82 & $<8$ & $<14$ & -- & $<10$ & $<1$ & 43 & 585 & $<.1$ \\
\hline 09-01-99 & 0810 & $<20$ & $<1$ & 733 & $<1.6$ & 82 & $<8$ & $<14$ & -- & $<30$ & $<1$ & 43 & 567 & $<.1$ \\
\hline \multicolumn{15}{|c|}{ MW4-1A } \\
\hline 06-02-99 & 0810 & M & 5 & 441 & $<1.6$ & 87 & $<8$ & $<14$ & -- & $<10$ & $<1$ & 41 & 1,240 & $<.1$ \\
\hline 08-31-99 & 1300 & $<20$ & 6 & 440 & $<1.6$ & 92 & $<8$ & $<14$ & -- & $<10$ & $<1$ & 44 & 1,240 & $<.1$ \\
\hline \multicolumn{15}{|c|}{ MW4-1B } \\
\hline 06-02-99 & 0915 & $<10$ & 1 & 1,130 & $<1.6$ & 83 & $<8$ & $<14$ & -- & $<10$ & $<1$ & 35 & 352 & $<.1$ \\
\hline 08-31-99 & 1350 & $<20$ & 2 & 1,020 & $<1.6$ & 82 & $<8$ & $<14$ & -- & $<10$ & $<1$ & 37 & 543 & $<.1$ \\
\hline \multicolumn{15}{|c|}{ MW4-2A } \\
\hline 06-02-99 & 1100 & $<10$ & 4 & 327 & $<1.6$ & 77 & $<8$ & $<14$ & -- & $<10$ & $<1$ & 36 & 1,710 & $<.1$ \\
\hline 08-31-99 & 1500 & $<20$ & 5 & 338 & $<1.6$ & 87 & $<8$ & $<14$ & -- & $<10$ & $<1$ & 38 & 1,860 & $<.1$ \\
\hline \multicolumn{15}{|c|}{ MW4-2B } \\
\hline 06-02-99 & 1020 & $<10$ & $<1$ & 1,080 & $<1.6$ & 99 & $<8$ & $<14$ & -- & $<10$ & $<1$ & 43 & 1,030 & $<.1$ \\
\hline 08-31-99 & 1600 & $<20$ & $<1$ & 1,090 & $<1.6$ & 106 & $<8$ & $<14$ & -- & $<30$ & $<1$ & 47 & 1,170 & $<.1$ \\
\hline \multicolumn{15}{|c|}{ USGS-8S } \\
\hline 06-04-99 & 0830 & $<10$ & $<1$ & 193 & $<1.6$ & 54 & $<8$ & $<14$ & $<7$ & $<10$ & $<1$ & 23 & 178 & $<.1$ \\
\hline \multicolumn{15}{|c|}{ USGS-8D } \\
\hline 06-04-99 & 0830 & $<10$ & $<1$ & 538 & $<1.6$ & 67 & $<8$ & $<14$ & 10 & $<10$ & $<1$ & 34 & 440 & $<.1$ \\
\hline \multicolumn{15}{|c|}{ USGS-9S } \\
\hline 06-03-99 & 1510 & $<10$ & 6 & 428 & $<1.6$ & 46 & $<8$ & $<14$ & $<7$ & $<10$ & $<1$ & E4 & 19.5 & $<.1$ \\
\hline \multicolumn{15}{|c|}{ USGS-9D } \\
\hline 06-03-99 & 1610 & $<10$ & $<1$ & 555 & $<1.6$ & 30 & $<8$ & $<14$ & -- & $<10$ & $<1$ & 7 & 316 & $<.1$ \\
\hline * & 1615 & $<10$ & $<1$ & 552 & $<1.6$ & 27 & $<8$ & $<14$ & -- & $<10$ & $<1$ & 8 & 319 & $<.1$ \\
\hline
\end{tabular}


Table 2. Concentrations of trace elements, organic carbon, bromide, and silica in samples from selected monitoring wells, 1999.-Continued

[ $\mu \mathrm{g} / \mathrm{L}$, micrograms per liter; constituent concentrations are dissolved, unless otherwise noted; <, less than; --, no data; E, estimated; mg/L, milligrams per liter; COD, chemical oxygen demand; *, replicate sample]

\begin{tabular}{|c|c|c|c|c|c|c|c|c|c|c|c|c|c|}
\hline Date & Time & $\begin{array}{l}\text { Molybdenum } \\
\quad(\mu \mathrm{g} / \mathrm{L})\end{array}$ & $\begin{array}{l}\text { Nickel } \\
(\mu \mathrm{g} / \mathrm{L})\end{array}$ & $\begin{array}{c}\text { Selenium } \\
(\mu \mathrm{g} / \mathrm{L})\end{array}$ & $\begin{array}{l}\text { Silver } \\
(\mu \mathbf{g} / \mathrm{L})\end{array}$ & $\begin{array}{c}\text { Strontium } \\
(\mu \mathbf{g} / \mathrm{L})\end{array}$ & $\begin{array}{c}\text { Vanadium } \\
(\mu \mathrm{g} / \mathrm{L})\end{array}$ & $\begin{array}{l}\text { Zinc } \\
(\mu \mathrm{g} / \mathrm{L})\end{array}$ & $\begin{array}{l}\text { Organic } \\
\text { carbon } \\
\text { (mg/L) }\end{array}$ & $\begin{array}{c}\text { Organic } \\
\text { carbon, } \\
\text { total } \\
(\mathrm{mg} / \mathrm{L})\end{array}$ & $\begin{array}{c}\text { COD, } \\
\text { high level } \\
\text { (mg/L) }\end{array}$ & $\begin{array}{c}\text { Bromide } \\
\text { (mg/L) }\end{array}$ & $\begin{array}{l}\text { Silica } \\
\text { (mg/L) }\end{array}$ \\
\hline \multicolumn{14}{|c|}{ MW2-1B } \\
\hline 06-02-99 & 1400 & $<50$ & $<40$ & $<1$ & $<4$ & 715 & $<10$ & $<20$ & 2.3 & 2.3 & 20 & 0.03 & 35.3 \\
\hline 08-27-99 & 0900 & $<30$ & $<40$ & $<1$ & $<7$ & 723 & $<10$ & $<20$ & 2.9 & 3.0 & $<10$ & .02 & 37.2 \\
\hline \multicolumn{14}{|c|}{ MW3-1A } \\
\hline 06-02-99 & 1440 & $<50$ & $<40$ & $<1$ & $<4$ & 796 & $<10$ & $<20$ & 1.8 & 1.3 & 10 & .06 & 22.4 \\
\hline 09-01-99 & 0900 & $<30$ & E20 & 1 & $<7$ & 848 & $<10$ & $<20$ & 2.5 & 2.5 & $<10$ & .07 & 24.7 \\
\hline \multicolumn{14}{|c|}{ MW3-1B } \\
\hline 06-02-99 & 1530 & $<50$ & $<40$ & $<1$ & $<4$ & 876 & $<10$ & $<20$ & 1.8 & 7.3 & 20 & .01 & 34.8 \\
\hline 09-01-99 & 0810 & $<30$ & $<40$ & $<1$ & $<7$ & 880 & $<10$ & $<20$ & 2.4 & 2.7 & $<10$ & $<.01$ & 35.3 \\
\hline \multicolumn{14}{|c|}{ MW4-1A } \\
\hline 06-02-99 & 0810 & $<50$ & $<40$ & $<1$ & $<4$ & 1,060 & $<10$ & $<20$ & 2.0 & 8.0 & 20 & .09 & 29.0 \\
\hline 08-31-99 & 1300 & $<30$ & $<40$ & $<1$ & $<7$ & 1,130 & $<10$ & $<20$ & 3.1 & 4.8 & $<10$ & .04 & 30.7 \\
\hline \multicolumn{14}{|c|}{ MW4-1B } \\
\hline 06-02-99 & 0915 & $<50$ & $<40$ & $<1$ & $<4$ & 792 & $<10$ & $<20$ & 2.2 & 2.8 & 20 & .10 & 33.2 \\
\hline 08-31-99 & 1350 & $<30$ & $<40$ & $<1$ & $<7$ & 819 & $<10$ & $<20$ & 2.6 & 3.4 & $<10$ & .10 & 35.6 \\
\hline \multicolumn{14}{|c|}{ MW4-2A } \\
\hline 06-02-99 & 1100 & $<50$ & $<40$ & $<1$ & $<4$ & 924 & $<10$ & $<20$ & 2.4 & 1.8 & 20 & .05 & 26.0 \\
\hline 08-31-99 & 1500 & $<30$ & E20 & $<1$ & $<7$ & 1,030 & $<10$ & $<20$ & 3.0 & 3.3 & $<10$ & .04 & 28.2 \\
\hline \multicolumn{14}{|c|}{ USGS-8S } \\
\hline 06-04-99 & 0830 & $<50$ & $<40$ & $<1$ & $<4$ & 746 & $<10$ & $<20$ & 1.3 & 1.4 & 20 & .01 & 23.1 \\
\hline \multicolumn{14}{|c|}{ USGS-8D } \\
\hline 06-04-99 & 0830 & $<50$ & $<40$ & $<1$ & $<4$ & 716 & $<10$ & $<20$ & 1.6 & 2.5 & 20 & .03 & 27.1 \\
\hline \multicolumn{14}{|c|}{ USGS-9S } \\
\hline 06-03-99 & 1510 & $<50$ & $<40$ & 1 & $<4$ & 272 & $<10$ & $<20$ & 1.1 & 1.4 & 10 & .02 & 20.9 \\
\hline \multicolumn{14}{|c|}{ USGS-9D } \\
\hline 06-03-99 & 1610 & $<50$ & $<40$ & $<1$ & $<4$ & 196 & $<10$ & $<20$ & 1.1 & 2.7 & 10 & .17 & 29.5 \\
\hline$*$ & 1615 & $<50$ & $<40$ & $<1$ & $<4$ & 196 & $<10$ & $<20$ & 1.0 & 1.5 & 20 & .06 & 29.6 \\
\hline
\end{tabular}


Table 3. Organic compounds and pesticides analyzed and minimum reporting limits for samples from selected monitoring wells, June 1999.

[--, not detected; $\mu \mathrm{g} / \mathrm{L}$, micrograms per liter; CFC, 1,1,2-trichloro-1,2,2-trifluorethane; p,p'-DDD, 1,1'-(2,2-dichloroethylidene)bis(4-chlorobenzene); p,p'-DDE, 1,1'-(dichloroethylidene)bis(4-chlorobenzene); p,p'-DDT, 1,1'-(2,2,2-dichloroethylidene)bis(4-chlorobenzene); PCBs, polychlorinated biphenyls; PCNs, polychlorinated naphthalenes]

\begin{tabular}{|c|c|c|c|c|c|}
\hline Constituent & $\begin{array}{c}\text { Minimum } \\
\text { reporting } \\
\text { limit } \\
(\mu \mathrm{g} / \mathrm{L})\end{array}$ & $\begin{array}{c}\text { Detected } \\
\text { in water } \\
\text { sample }\end{array}$ & Constituent & $\begin{array}{l}\text { Minimum } \\
\text { reporting } \\
\text { limit } \\
(\mu \mathrm{g} / \mathrm{L})\end{array}$ & $\begin{array}{c}\text { Detected } \\
\text { in water } \\
\text { sample }\end{array}$ \\
\hline \multicolumn{6}{|c|}{ Organic compounds } \\
\hline 1,1-Dichloroethane & 3 & -- & 4-Isoppropyl toluene & 3 & -- \\
\hline 1,1-Dichloroethene & 3 & -- & 4-Nitrophenol & 30 & -- \\
\hline 1,1-Dichloropropene & 3 & -- & 9H-Fluorene & 5 & -- \\
\hline 1,1,1-Trichloroethane & 3 & -- & Acenaphthene & 5 & -- \\
\hline 1,1,1,2-Tetrachloroethane & 3 & -- & Acenaphthylene & 5 & -- \\
\hline 1,1,2-Trichloroethane & 3 & -- & Acrylonitrile & 2.5 & -- \\
\hline 1,1,2,2-Tetrachloroethane & 3 & -- & Anthracene & 5 & -- \\
\hline 1,2-Dibromoethane & 3 & -- & Benzene & 3 & -- \\
\hline 1,2-Dichlorobenzene & 3 & -- & Benzidine & 40 & -- \\
\hline 1,2-Dichloroethane & 3 & -- & Benzo[a]anthracene & 10 & -- \\
\hline 1,2-Dichloropropane & 3 & -- & Benzo[a]pyrene & 10 & -- \\
\hline 1,2-Diphenylhydrazine & 5 & -- & Benzo[b]fluoranthene & 10 & -- \\
\hline 1,2,3-Trichlorobenzene & 3 & -- & Benzo[g,h,i]perylene & 10 & -- \\
\hline 1,2,3-Trichloropropane & 3 & -- & Benzo[k]fluoranthene & 10 & -- \\
\hline 1,2,4-Trichlorobenzene & 3 & -- & Benzyl-n-butyl phthalate & 5 & -- \\
\hline 1,2,4-Trimethylbenzene & 3 & -- & Bis(2-chloroethoxy) methane & 5 & -- \\
\hline 1,3-Dichlorobenzene & 3 & -- & Bis(2-chloroethyl) ether & 5 & -- \\
\hline 1,3-Dichloropropane & 3 & -- & Bis(2-chloroisopropyl) ether & 5 & -- \\
\hline 1,3,5-Trimethylbenzene & 3 & -- & Bis(2-ethylhexyl) phthalate & 5 & -- \\
\hline 1,4-Dichlorobenzene & 3 & -- & Bromobenzene & 3 & -- \\
\hline 2-Chloronaphthalene & 5 & -- & Bromochloromethane & 3 & -- \\
\hline 2-Chlorophenol & 5 & -- & Bromodichloromethane & 3 & -- \\
\hline 2-Chlorotoluene & 3 & -- & Bromomethane & 3 & -- \\
\hline 2-Methyl-4,6-dinitrophenol & 30 & -- & CFC-113 & 3 & -- \\
\hline 2-Nitrophenol & 5 & -- & Chrysene & 10 & -- \\
\hline 2,2-Dichloropropane & 3 & -- & Chlorobenzene & 3 & -- \\
\hline 2,4-Dichlorophenol & 5 & -- & Chloroethane & 3 & -- \\
\hline 2,4-Dimethylphenol & 5 & -- & Chloromethane & 3 & -- \\
\hline 2,4-Dinitrophenol & 20 & -- & cis-1,2-Dichloroethene & 3 & -- \\
\hline 2,4-Dinitrotoluene & 5 & -- & cis-1,3-Dichloropropene & 3 & -- \\
\hline 2,4,6-Trichlorophenol & 20 & -- & Dibenzo[a,h]anthracene & 10 & -- \\
\hline 2,6-Dinitrotoluene & 5 & -- & Dibromochloromethane & 3 & -- \\
\hline 3,3'-Dichlorobenzidine & 20 & -- & Dibromochloropropane & 3 & -- \\
\hline 4-Bromophenyl phenyl ether & 5 & -- & Dibromomethane & 3 & -- \\
\hline 4-Chloro-3-methylphenol & 30 & -- & Dichlorodifluoromethane & 3 & -- \\
\hline 4-Chlorophenyl phenyl ether & 5 & -- & Dichloromethane & 3 & -- \\
\hline 4-Chlorotoluene & 3 & -- & Diethyl phthalate & 5 & -- \\
\hline
\end{tabular}


Table 3. Organic compounds and pesticides analyzed and minimum reporting limits for samples from selected monitoring wells, June 1999.-Continued

[--, not detected; $\mu \mathrm{g} / \mathrm{L}$, micrograms per liter; CFC, 1,1,2-trichloro-1,2,2-trifluorethane; p,p'-DDD, 1,1'-(2,2-dichloroethylidene)bis(4-chlorobenzene); p,p'-DDE, 1,1'-(dichloroethylidene)bis(4-chlorobenzene); p,p'-DDT, 1,1'-(2,2,2-dichloroethylidene)bis(4-chlorobenzene); PCBs, polychlorinated biphenyls; PCNs, polychlorinated naphthalenes]

\begin{tabular}{|c|c|c|c|c|c|}
\hline Constituent & $\begin{array}{l}\text { Minimum } \\
\text { reporting } \\
\text { limit } \\
(\mu \mathrm{g} / \mathrm{L})\end{array}$ & $\begin{array}{l}\text { Detected } \\
\text { in water } \\
\text { sample }\end{array}$ & Constituent & $\begin{array}{c}\text { Minimum } \\
\text { reporting } \\
\text { limit } \\
(\mu \mathrm{g} / \mathrm{L})\end{array}$ & $\begin{array}{c}\text { Detected } \\
\text { in water } \\
\text { sample }\end{array}$ \\
\hline \multicolumn{6}{|c|}{ Organic compounds_-Continued } \\
\hline Dimethyl phthalate & 5 & -- & N-Nitrosodiphenylamine & 5 & -- \\
\hline Di-n-butyl phthalate & 5 & -- & Pentachlorophenol & 30 & -- \\
\hline Di-n-octyl phthalate & 10 & -- & Pentanthrene & 5 & -- \\
\hline Ethylbenzene & 3 & -- & Phenol & 5 & -- \\
\hline Fluoranthene & 5 & -- & Pyrene & 5 & -- \\
\hline Hexachlorobenzene & 5 & -- & sec-Butylbenzene & 3 & -- \\
\hline Hexachlorobutadiene & 3 & -- & Styrene & 3 & -- \\
\hline Hexachlorocyclopentadiene & 20 & -- & tert-Butylbenzene & 3 & -- \\
\hline Hexachloroethane & 5 & -- & Tetrachloroethene & 3 & -- \\
\hline Indeno[1,2,3-cd]pyrene & 10 & -- & Tetrachloromethane & 3 & -- \\
\hline Isophorone & 5 & -- & Toluene & 3 & -- \\
\hline Isopropylbenzene & 3 & -- & trans-1,2-Dichloroethene & 3 & -- \\
\hline Methyl-t-butyl ether & 3 & -- & trans-1,3-Dichloropropene & 3 & -- \\
\hline Naphthalene & 3 & -- & Tribromomethane & 3 & -- \\
\hline Nitrobenzene & 5 & -- & Trichloroethene & 3 & -- \\
\hline n-Butylbenzene & 3 & -- & Trichlorofluoromethane & 3 & -- \\
\hline n-Propylbenzene & 3 & -- & Trichloromethane & 3 & -- \\
\hline $\mathrm{N}$-Nitrosodimethylamine & 5 & -- & Vinyl chloride & 1 & -- \\
\hline N-Nitrosodi-n-propylamine & 5 & -- & Xylenes (total) & 3 & -- \\
\hline \multicolumn{6}{|c|}{ Pesticides } \\
\hline Aldrin & .01 & -- & p,p'-DDD & .01 & -- \\
\hline alpha-Endosulfan & .01 & -- & p,p'-DDE & .01 & -- \\
\hline Chlordane & .1 & -- & $\mathrm{p}, \mathrm{p}$-DDT & .01 & -- \\
\hline Dieldrin & .010 & -- & p,p'-Ethyl-DDD & .1 & -- \\
\hline Endrin & .01 & -- & p,p'-Methoxychlor & .01 & -- \\
\hline Heptachlor & .01 & -- & PCBs & .1 & -- \\
\hline Heptachlor epoxide & .01 & -- & PCNs & .1 & -- \\
\hline Lindane & .01 & -- & Toxaphene & 1 & -- \\
\hline Mirex & .01 & -- & & & \\
\hline
\end{tabular}


Table 4. Wastewater organic compounds analyzed and minimum reporting limits for samples from selected monitoring wells and surface-water sites, 1999-2003.

[--, not detected; $\mu \mathrm{g} / \mathrm{L}$, micrograms per liter; AHTN, acetyl hexamethyl tetrahydronaphthalene; DEET, N,N-diethyl-meta-toluamide; HHCB, hexahydrohexamethylcyclopentabenzopyran; compounds detected are indicated with an $\mathrm{X}$ in the table]

\begin{tabular}{|c|c|c|c|c|c|}
\hline Constituent & $\begin{array}{c}\text { Minimum } \\
\text { reporting } \\
\quad \text { limit } \\
(\mu \mathrm{g} / \mathrm{L})\end{array}$ & $\begin{array}{l}\text { Detected in } \\
\text { ground or } \\
\text { surface water } \\
\text { (table 6) }\end{array}$ & Constituent & $\begin{array}{c}\text { Minimum } \\
\text { reporting } \\
\quad \text { limit } \\
(\mu \mathrm{g} / \mathrm{L})\end{array}$ & $\begin{array}{l}\text { Detected in } \\
\text { ground or } \\
\text { surface water } \\
\text { (table 6) }\end{array}$ \\
\hline 1,4-Dichlorobenzene & 0.5 & $\mathrm{X}$ & Dichlorvos & 1 & -- \\
\hline 1-Methylnaphthalene & .5 & -- & Diethoxynonylphenol & 5 & $\mathrm{X}$ \\
\hline 2,6-Dimethylnaphthalene & .5 & -- & Diethoxyoctylphenol & 1 & $\mathrm{X}$ \\
\hline 2-Methylnaphthalene & .5 & -- & d-Limonene & .5 & -- \\
\hline 3-beta-Coprostanol & 2 & $\mathrm{X}$ & Ethoxyoctylphenol & .5 & $\mathrm{X}$ \\
\hline 3-Methyl-1H-indole & 1 & $\mathrm{X}$ & HHCB & .5 & $\mathrm{X}$ \\
\hline 3-tert-Butyl-4-hydroxyanisole (BHA) & 5 & -- & Indole & .5 & $\mathrm{X}$ \\
\hline 4-Cumylphenol & 1 & -- & Isoborneol & .5 & -- \\
\hline 4-Octylphenol & 1 & -- & Isophorone & .5 & $\mathrm{X}$ \\
\hline 4-Nonylphenol & 5 & $\mathrm{X}$ & Isopropylbenzene & .5 & -- \\
\hline 4-tert-Octylphenol & 1 & $\mathrm{X}$ & Isoquinoline & .5 & -- \\
\hline 5-Methyl-1H-benzotriazole & 2 & $\mathrm{X}$ & Menthol & .5 & $\mathrm{X}$ \\
\hline 9,10-Anthraquinone & .5 & -- & Metalaxyl & .5 & -- \\
\hline Acetophenone & .5 & $\mathrm{X}$ & Methyl salicylate & .5 & $\mathrm{X}$ \\
\hline AHTN & .5 & $\mathrm{X}$ & Metolachlor & .5 & -- \\
\hline Anthracene & .5 & -- & Naphthalene & .5 & -- \\
\hline Benzo[a]pyrene & .5 & -- & p-Cresol & 1 & $\mathrm{X}$ \\
\hline Benzophenone & .5 & $\mathrm{X}$ & Pentachlorophenol & 2 & -- \\
\hline beta-Sitosterol & 2 & $\mathrm{X}$ & Phenanthrene & .5 & -- \\
\hline beta-Stigmastanol & 2 & $\mathrm{X}$ & Phenol & .5 & $\mathrm{X}$ \\
\hline Bisphenol A & 1 & $\mathrm{X}$ & Prometon & .5 & $\mathrm{X}$ \\
\hline Bromacil & .5 & -- & Pyrene & .5 & -- \\
\hline Caffeine & .5 & $\mathrm{X}$ & Tetrachloroethene & .5 & -- \\
\hline Camphor & .5 & $\mathrm{X}$ & Tribromomethane & .5 & $\mathrm{X}$ \\
\hline Carbaryl & 1 & $\mathrm{X}$ & Tributyl phosphate & .5 & $\mathrm{X}$ \\
\hline Carbazole & .5 & -- & Triclosan & 1 & $\mathrm{X}$ \\
\hline Chlorpyrifos & .5 & -- & Triethyl citrate & .5 & $\mathrm{X}$ \\
\hline Cholesterol & 2 & $\mathrm{X}$ & Triphenyl phosphate & .5 & $\mathrm{X}$ \\
\hline Cotinine & 1 & $\mathrm{X}$ & Tris(2-butoxyethyl) phosphate & .5 & $\mathrm{X}$ \\
\hline DEET & .5 & $\mathrm{X}$ & Tris(2-chloroethyl) phosphate & .5 & $\mathrm{X}$ \\
\hline Diazinon & .5 & $\mathrm{X}$ & Tris(dichloroisopropyl) phosphate & .5 & $\mathrm{X}$ \\
\hline
\end{tabular}


Table 5. Pesticides analyzed and minimum reporting limits for samples from selected monitoring wells and surface-water sites, 1999-2003.

[--, not detected; $\mu \mathrm{g} / \mathrm{L}$, micrograms per liter; CIAT, 2-chloro-4-isopropylamino-6-amino-s-triazine; HCH, hexachlorocyclohexane; DCPA, dimethyl tetrachloroterephthalate; EPTC, s-ethyl dipropylthiocarbamate; DDE, dichlorodiphenyl dichloroethylene; compounds detected are indicated with an $\mathrm{X}$ in the table]

\begin{tabular}{|c|c|c|c|c|c|}
\hline Constituent & $\begin{array}{c}\text { Minimum } \\
\text { reporting } \\
\text { limit } \\
(\mu \mathrm{g} / \mathrm{L})\end{array}$ & $\begin{array}{l}\text { Detected in } \\
\text { ground or } \\
\text { surface water } \\
\text { (table 6) }\end{array}$ & Constituent & $\begin{array}{c}\text { Minimum } \\
\text { reporting } \\
\text { limit } \\
(\mu \mathrm{g} / \mathrm{L})\end{array}$ & $\begin{array}{l}\text { Detected in } \\
\text { ground or } \\
\text { surface water } \\
\text { (table 6) }\end{array}$ \\
\hline 2,6-Diethylanaline & 0.006 & -- & Malathion & 0.027 & -- \\
\hline CIAT & .014 & $\mathrm{X}$ & Methyl parathion & .015 & -- \\
\hline Acetochlor & .006 & -- & Metolachlor & .006 & $\mathrm{X}$ \\
\hline Alachlor & .005 & -- & Metribuzin & .028 & $\mathrm{X}$ \\
\hline alpha-HCH & .005 & -- & Molinate & .003 & -- \\
\hline Atrazine & .007 & $X$ & Napropamide & .007 & -- \\
\hline Azinphos-methyl & .05 & -- & $\mathrm{p}, \mathrm{p}$-DDE & .003 & -- \\
\hline Benfluralin & .01 & -- & Parathion & .01 & -- \\
\hline Butylate & .004 & -- & Pebulate & .004 & -- \\
\hline Carbaryl & .041 & $\mathrm{X}$ & Pendimethalin & .022 & -- \\
\hline Carbofuran & .02 & -- & Phorate & .055 & -- \\
\hline Chlorpyrifos & .005 & -- & Prometon & .010 & $\mathrm{X}$ \\
\hline cis-Permethrin & .006 & -- & Propyzamide & .004 & -- \\
\hline Cyanazine & .018 & -- & Propachlor & .010 & -- \\
\hline DCPA & .003 & -- & Propanil & .011 & -- \\
\hline Dieldrin & .009 & -- & Propargite & .023 & -- \\
\hline Disulfoton & .021 & -- & Simazine & .005 & -- \\
\hline EPTC & .004 & -- & Tebuthiuron & .016 & -- \\
\hline Ethalfluralin & .009 & -- & Terbacil & .034 & -- \\
\hline Ethoprop & .012 & -- & Terbufos & .017 & -- \\
\hline Fonofos & .0053 & -- & Thiobencarb & .010 & -- \\
\hline Lindane & .004 & -- & Triallate & .006 & -- \\
\hline Linuron & .035 & -- & Trifluralin & .009 & -- \\
\hline
\end{tabular}


Table 6. Concentrations of wastewater organic compounds and pesticides in samples from selected monitoring wells and surface-water sampling sites, $1999-2003$.

$[\mu \mathrm{g} / \mathrm{L}$, micrograms per liter; constituent concentrations are dissolved, unless otherwise noted; CIAT, 2-chloro-4-isopropylamino-6-amino-s-triazine; AHTN, acetyl hexamethyl tetrahydronaphthalene; --, concentration less than reporting limit; nd, not determined; E, estimated; M, presence verified, but not quantified; *, replicate sample; DEET, n,n-diethyl-meta-toluamide]

\begin{tabular}{|c|c|c|c|c|c|c|c|c|c|c|c|c|c|c|c|}
\hline Date & Time & $\begin{array}{c}\text { 1,4,Dichloro- } \\
\text { benzene } \\
(\mu \mathrm{g} / \mathrm{L})\end{array}$ & $\begin{array}{c}\text { 3-beta- } \\
\text { Coprostanol } \\
(\mu \mathrm{g} / \mathbf{L})\end{array}$ & $\begin{array}{c}\text { 3-Methyl-1H- } \\
\text { indole } \\
(\mu \mathrm{g} / \mathrm{L})\end{array}$ & $\begin{array}{l}\text { 4-Nonyl- } \\
\text { phenol } \\
(\mu \mathrm{g} / \mathrm{L})\end{array}$ & $\begin{array}{l}\text { 4-tert-0ctyl- } \\
\text { phenol } \\
(\mu \mathrm{g} / \mathrm{L})\end{array}$ & $\begin{array}{c}\text { 5-Methyl-1H- } \\
\text { benzotriazole } \\
(\mu \mathrm{g} / \mathrm{L})\end{array}$ & $\begin{array}{c}\text { CIAT } \\
(\mu \mathrm{g} / \mathbf{L})\end{array}$ & $\begin{array}{l}\text { Aceto- } \\
\text { phenone } \\
(\mu \mathrm{g} / \mathrm{L})\end{array}$ & $\begin{array}{c}\text { AHTN } \\
(\mu \mathrm{g} / \mathrm{L})\end{array}$ & $\begin{array}{c}\text { Atrazine } \\
(\mu \mathrm{g} / \mathrm{L})\end{array}$ & $\begin{array}{c}\text { Benzo- } \\
\text { phenone } \\
(\mu \mathrm{g} / \mathrm{L})\end{array}$ & $\begin{array}{c}\text { beta- } \\
\text { Sitosterol } \\
(\mu \mathrm{g} / \mathrm{L})\end{array}$ & $\begin{array}{c}\text { beta- } \\
\text { Stigmastanol } \\
(\mu \mathrm{g} / \mathrm{L})\end{array}$ & $\begin{array}{c}\text { Bisphenol } \\
\text { A } \\
(\mu \mathrm{g} / \mathrm{L})\end{array}$ \\
\hline \multicolumn{16}{|c|}{ MW1-1A } \\
\hline 04-03-03 & 1040 & -- & -- & -- & -- & -- & -- & nd & -- & -- & nd & E0.1 & -- & -- & -- \\
\hline $08-28-03$ & 1000 & -- & -- & -- & -- & -- & -- & nd & -- & -- & nd & -- & -- & -- & -- \\
\hline \multicolumn{16}{|c|}{ MW1-2A } \\
\hline 04-03-03 & 1000 & -- & -- & -- & -- & -- & -- & nd & -- & -- & nd & E.2 & -- & -- & -- \\
\hline $08-28-03$ & 1100 & -- & -- & -- & -- & -- & -- & nd & -- & -- & nd & -- & -- & -- & M \\
\hline \multicolumn{16}{|c|}{ MW1-3A } \\
\hline 04-03-03 & 1630 & -- & -- & -- & -- & -- & -- & nd & E. 2 & -- & nd & E.1 & -- & -- & -- \\
\hline $08-28-03$ & 1140 & -- & -- & M & -- & -- & -- & nd & -- & -- & nd & -- & -- & -- & -- \\
\hline \multicolumn{16}{|c|}{ MW1-3B } \\
\hline 06-03-99 & 1350 & -- & nd & nd & nd & nd & nd & -- & nd & nd & 0.008 & nd & nd & nd & nd \\
\hline \multicolumn{16}{|c|}{ MW1-4A } \\
\hline 04-03-03 & 1530 & -- & -- & -- & -- & -- & -- & -- & -- & -- & -- & -- & -- & -- & -- \\
\hline $08-28-03$ & 0920 & -- & -- & -- & -- & -- & -- & -- & -- & -- & -- & -- & -- & -- & -- \\
\hline \multicolumn{16}{|c|}{ MW3-1A } \\
\hline 04-01-03 & 1650 & -- & -- & -- & E1 & -- & -- & nd & E. 2 & -- & nd & E. 2 & -- & -- & -- \\
\hline $08-27-03$ & 1430 & -- & -- & -- & -- & -- & -- & nd & -- & -- & nd & -- & -- & -- & -- \\
\hline \multicolumn{16}{|c|}{ MW4-1A } \\
\hline $04-02-03$ & 0950 & -- & -- & -- & -- & -- & -- & -- & E. 2 & -- & .007 & E. 2 & -- & -- & -- \\
\hline $08-27-03$ & 1230 & -- & -- & -- & -- & -- & -- & -- & -- & -- & -- & -- & -- & -- & -- \\
\hline \multicolumn{16}{|c|}{ MW4-2A } \\
\hline $04-02-03$ & 0850 & -- & -- & -- & -- & -- & -- & nd & -- & -- & nd & M & -- & -- & -- \\
\hline $08-27-03$ & 1130 & -- & -- & -- & -- & -- & -- & nd & -- & -- & nd & -- & -- & -- & -- \\
\hline \multicolumn{16}{|c|}{ USGS-1 } \\
\hline $03-01-01$ & 1130 & nd & nd & nd & nd & nd & nd & E0.002 & nd & nd & E.002 & nd & nd & nd & nd \\
\hline \multicolumn{16}{|c|}{ USGS-3S } \\
\hline 04-01-03 & 1320 & -- & -- & -- & -- & -- & -- & nd & E. 2 & -- & nd & E. 1 & -- & -- & M \\
\hline \multicolumn{16}{|c|}{ USGS-9S } \\
\hline $02-20-01$ & 1330 & nd & nd & nd & nd & nd & nd & E.004 & nd & nd & -- & nd & nd & nd & nd \\
\hline
\end{tabular}


$[\mu \mathrm{g} / \mathrm{L}$, micrograms per liter; constituent concentrations are dissolved, unless otherwise noted; CIAT, 2-chloro-4-isopropylamino-6-amino-s-triazine; AHTN, acetyl hexamethyl tetrahydronaphthalene; --, concentration less than reporting limit; nd, not determined; E, estimated; M, presence verified, but not quantified; *, replicate sample; DEET, n,n-diethyl-meta-toluamide]

\begin{tabular}{|c|c|c|c|c|c|c|c|c|c|c|c|c|c|c|}
\hline Date & $\begin{array}{c}\text { Caffeine } \\
(\mu \mathrm{g} / \mathrm{L})\end{array}$ & $\begin{array}{c}\text { Camphor } \\
(\mu \mathrm{g} / \mathrm{L})\end{array}$ & $\begin{array}{l}\text { Carbaryl } \\
(\mu \mathrm{g} / \mathrm{L})\end{array}$ & $\begin{array}{c}\text { Cholesterol } \\
(\mu \mathrm{g} / \mathrm{L})\end{array}$ & $\begin{array}{c}\text { Cotinine } \\
(\mu \mathrm{g} / \mathrm{L})\end{array}$ & $\begin{array}{c}\text { DEET } \\
(\mu \mathrm{g} / \mathrm{L})\end{array}$ & $\begin{array}{c}\text { Diazinon } \\
(\mu \mathrm{g} / \mathrm{L})\end{array}$ & $\begin{array}{c}\text { Diethoxy- } \\
\text { nonylphenol } \\
(\mu \mathrm{g} / \mathrm{L})\end{array}$ & $\begin{array}{l}\text { Diethoxy- } \\
\text { octylphenol } \\
(\mu \mathrm{g} / \mathrm{L})\end{array}$ & $\begin{array}{c}\text { Ethoxy- } \\
\text { octylphenol } \\
(\mu \mathrm{g} / \mathrm{L})\end{array}$ & $\begin{array}{l}\text { HHCB } \\
(\mu \mathrm{g} / \mathrm{L})\end{array}$ & $\begin{array}{l}\text { Indole } \\
(\mu \mathrm{g} / \mathrm{L})\end{array}$ & $\begin{array}{c}\text { Isophorone } \\
(\mu \mathrm{g} / \mathrm{L})\end{array}$ & $\begin{array}{c}\text { Menthol } \\
(\mu \mathrm{g} / \mathrm{L})\end{array}$ \\
\hline \multicolumn{15}{|c|}{ MW1-1A } \\
\hline 04-03-02 & -- & -- & -- & -- & -- & E0.1 & -- & -- & -- & -- & -- & -- & -- & -- \\
\hline $08-28-02$ & -- & -- & -- & -- & -- & -- & $\begin{array}{l}-- \\
\text { MW1-2A }\end{array}$ & -- & -- & -- & -- & -- & -- & -- \\
\hline 04-03-02 & -- & -- & -- & -- & -- & E. 2 & -- & -- & -- & -- & -- & -- & -- & -- \\
\hline $08-28-02$ & -- & -- & -- & -- & -- & E. 1 & $\begin{array}{l}-- \\
\text { MW1-3A }\end{array}$ & -- & -- & -- & -- & -- & -- & -- \\
\hline 04-03-02 & -- & -- & -- & -- & -- & E.1 & -- & -- & -- & -- & -- & -- & -- & -- \\
\hline 08-28-02 & -- & -- & -- & -- & -- & -- & $\begin{array}{l}-- \\
\text { MW1-3B }\end{array}$ & -- & -- & -- & -- & -- & -- & -- \\
\hline 06-03-99 & nd & nd & -- & nd & nd & nd & MW1-4A & nd & nd & nd & nd & nd & -- & nd \\
\hline 04-03-02 & -- & -- & -- & -- & -- & -- & -- & -- & -- & -- & -- & -- & -- & -- \\
\hline $08-28-02$ & -- & -- & -- & -- & -- & -- & $\begin{array}{l}-- \\
\text { MW3-1A }\end{array}$ & -- & -- & -- & -- & -- & -- & -- \\
\hline 04-01-02 & -- & -- & -- & -- & -- & E.1 & -- & -- & -- & -- & -- & -- & -- & -- \\
\hline $08-27-02$ & -- & -- & -- & -- & -- & -- & MW4-1A & -- & -- & -- & -- & -- & -- & -- \\
\hline $04-02-03$ & -- & -- & -- & -- & -- & E. 2 & -- & -- & -- & -- & -- & -- & -- & -- \\
\hline $08-27-03$ & -- & -- & -- & -- & -- & -- & $\begin{array}{l}-- \\
\text { MW4-2A }\end{array}$ & -- & -- & -- & -- & -- & -- & -- \\
\hline $04-02-03$ & -- & -- & -- & -- & -- & E.1 & -- & -- & -- & -- & -- & -- & -- & -- \\
\hline $08-27-03$ & -- & -- & -- & -- & -- & M & USGS-1 & -- & -- & -- & -- & -- & -- & -- \\
\hline 03-01-01 & nd & nd & -- & nd & nd & nd & $\begin{array}{l}-- \\
\text { USGS-3S }\end{array}$ & nd & nd & nd & nd & nd & nd & nd \\
\hline 04-01-03 & -- & -- & -- & -- & -- & E.1 & $\begin{array}{l}-- \\
\text { USGS-9S }\end{array}$ & -- & -- & -- & -- & -- & -- & -- \\
\hline $02-20-01$ & nd & nd & -- & nd & nd & nd & -- & nd & nd & nd & nd & nd & -- & nd \\
\hline
\end{tabular}


Table 6. Concentrations of wastewater organic compounds and pesticides in samples from selected monitoring wells and surface-water sampling sites, 1999-2003.-Continued [ $\mu \mathrm{g} / \mathrm{L}$, micrograms per liter; constituent concentrations are dissolved, unless otherwise noted; CIAT, 2-chloro-4-isopropylamino-6-amino-s-triazine; AHTN, acetyl hexamethyl tetrahydronaphthalene; --, concentration less than reporting limit; nd, not determined; E, estimated; M, presence verified, but not quantified; *, replicate sample; DEET, n,n-diethyl-meta-toluamide

\begin{tabular}{|c|c|c|c|c|c|c|c|c|c|c|c|c|c|c|c|}
\hline Date & $\begin{array}{c}\text { Methyl } \\
\text { salicylate } \\
(\mu \mathrm{g} / \mathbf{L})\end{array}$ & $\begin{array}{c}\text { Metolachlor } \\
(\mu \mathrm{g} / \mathrm{L})\end{array}$ & $\begin{array}{c}\text { Metribuzin } \\
(\mu \mathrm{g} / \mathrm{L})\end{array}$ & $\begin{array}{c}\mathrm{p} \text {-Cresol } \\
(\mu \mathrm{g} / \mathrm{L})\end{array}$ & $\begin{array}{l}\text { Phenol } \\
(\mu \mathrm{g} / \mathrm{L})\end{array}$ & $\begin{array}{c}\text { Prometon } \\
(\mu \mathrm{g} / \mathrm{L})\end{array}$ & $\underset{(\mu \mathrm{g} / \mathrm{L})}{\text { Simazine }}$ & $\begin{array}{c}\text { Tribromo- } \\
\text { methane } \\
(\mu \mathrm{g} / \mathrm{L})\end{array}$ & $\begin{array}{c}\text { Tributyl } \\
\text { phosphate } \\
(\mu \mathrm{g} / \mathrm{L})\end{array}$ & $\begin{array}{c}\text { Triclosan } \\
(\mu \mathrm{g} / \mathrm{L})\end{array}$ & $\begin{array}{l}\text { Triethyl } \\
\text { citrate } \\
(\mu \mathrm{g} / \mathrm{L})\end{array}$ & $\begin{array}{c}\text { Triphenyl } \\
\text { phosphate } \\
(\mu \mathrm{g} / \mathrm{L})\end{array}$ & $\begin{array}{c}\text { Tris } \\
\text { (2-butoxy- } \\
\text { ethyl) } \\
\text { phosphate } \\
(\mu \mathrm{g} / \mathrm{L})\end{array}$ & $\begin{array}{c}\text { Tris } \\
\text { (2-chloro- } \\
\text { ethyl) } \\
\text { phosphate } \\
(\mu \mathrm{g} / \mathrm{L})\end{array}$ & $\begin{array}{c}\text { Tris } \\
\text { (dichloro- } \\
\text { i-Pr) } \\
\text { phosphate } \\
(\mu \mathrm{g} / \mathrm{L})\end{array}$ \\
\hline \multicolumn{16}{|c|}{ MW1-1A } \\
\hline 04-03-02 & -- & -- & nd & -- & 1.0 & -- & nd & -- & -- & -- & -- & -- & -- & -- & -- \\
\hline 08-28-02 & -- & -- & nd & -- & 1.0 & -- & nd & -- & -- & -- & -- & -- & -- & -- & -- \\
\hline \multicolumn{16}{|c|}{ MW1-2A } \\
\hline 06-03-99 & M & -- & nd & -- & E. 3 & -- & nd & -- & -- & -- & -- & -- & -- & -- & -- \\
\hline 09-02-99 & -- & -- & nd & -- & -- & -- & nd & -- & -- & -- & -- & -- & -- & -- & -- \\
\hline \multicolumn{16}{|c|}{ MW1-3A } \\
\hline 04-03-02 & M & -- & nd & -- & E. 3 & -- & nd & -- & -- & -- & -- & -- & -- & -- & -- \\
\hline $08-28-02$ & -- & -- & nd & M & .8 & -- & nd & -- & -- & -- & -- & -- & -- & -- & -- \\
\hline \multicolumn{16}{|c|}{ MW1-3B } \\
\hline 06-03-99 & nd & -- & -- & nd & -- & -- & -- & nd & nd & nd & nd & nd & nd & nd & nd \\
\hline \multicolumn{16}{|c|}{ MW1-4A } \\
\hline 04-03-02 & -- & -- & -- & -- & 1.8 & -- & -- & -- & -- & -- & -- & -- & -- & -- & -- \\
\hline 08-28-02 & -- & -- & -- & -- & -- & -- & -- & -- & -- & -- & -- & -- & -- & -- & -- \\
\hline \multicolumn{16}{|c|}{ MW3-1A } \\
\hline 04-01-02 & E0.1 & -- & nd & -- & 1.2 & -- & nd & -- & -- & -- & -- & -- & -- & -- & -- \\
\hline $08-27-02$ & -- & -- & nd & -- & E. 3 & -- & nd & -- & -- & -- & -- & -- & -- & -- & -- \\
\hline \multicolumn{16}{|c|}{ MW4-1A } \\
\hline $04-02-03$ & E.1 & -- & -- & -- & E. 3 & -- & -- & -- & -- & -- & -- & -- & -- & -- & -- \\
\hline $08-27-03$ & -- & -- & -- & -- & .6 & -- & -- & -- & -- & -- & -- & -- & -- & -- & -- \\
\hline \multicolumn{16}{|c|}{ MW4-2A } \\
\hline $04-02-03$ & E.1 & -- & nd & -- & 1.8 & -- & nd & -- & -- & -- & -- & -- & -- & -- & -- \\
\hline $08-27-03$ & -- & -- & nd & -- & .6 & -- & nd & -- & -- & -- & -- & -- & -- & -- & -- \\
\hline \multicolumn{16}{|c|}{ USGS-1 } \\
\hline $03-01-01$ & nd & -- & -- & nd & nd & -- & -- & nd & nd & nd & nd & nd & nd & nd & nd \\
\hline \multicolumn{16}{|c|}{ USGS-3S } \\
\hline 04-01-03 & E. 2 & -- & nd & -- & .5 & -- & nd & -- & -- & -- & -- & -- & -- & -- & -- \\
\hline \multicolumn{16}{|c|}{ USGS-9S } \\
\hline $02-20-01$ & nd & -- & -- & nd & -- & -- & -- & nd & nd & nd & nd & nd & nd & nd & nd \\
\hline
\end{tabular}


Table 6. Concentrations of wastewater organic compounds and pesticides in samples from selected monitoring wells and surface-water sampling sites, 1999-2003.—Continued

$[\mu \mathrm{g} / \mathrm{L}$, micrograms per liter; constituent concentrations are dissolved, unless otherwise noted; CIAT, 2-chloro-4-isopropylamino-6-amino-s-triazine; AHTN, acetyl hexamethyl tetrahydronaphthalene; --, concentration less than reporting limit; nd, not determined; E, estimated; M, presence verified, but not quantified; *, replicate sample; DEET, n,n-diethyl-meta-toluamide]

\begin{tabular}{|c|c|c|c|c|c|c|c|c|c|c|c|c|c|c|c|}
\hline Date & Time & $\begin{array}{l}\text { 1,4,Dichloro- } \\
\text { benzene } \\
(\mu \mathrm{g} / \mathrm{L})\end{array}$ & $\begin{array}{c}\text { 3-beta- } \\
\text { Coprostanol } \\
(\mu \mathrm{g} / \mathrm{L})\end{array}$ & $\begin{array}{c}\text { 3-Methyl-1H- } \\
\text { indole } \\
(\mu \mathrm{g} / \mathrm{L})\end{array}$ & $\begin{array}{l}\text { 4-Nonyl- } \\
\text { phenol } \\
(\mu \mathrm{g} / \mathrm{L})\end{array}$ & $\begin{array}{l}\text { 4-tert-0ctyl- } \\
\text { phenol } \\
(\mu \mathrm{g} / \mathrm{L})\end{array}$ & $\begin{array}{c}\text { 5-Methyl-1H- } \\
\text { benzotriazole } \\
(\mu \mathrm{g} / \mathrm{L})\end{array}$ & $\underset{(\mu \mathrm{g} / \mathbf{L})}{\text { CIAT }}$ & $\begin{array}{c}\text { Aceto- } \\
\text { phenone } \\
(\mu \mathrm{g} / \mathrm{L})\end{array}$ & $\begin{array}{l}\text { AHTN } \\
(\mu \mathrm{g} / \mathbf{L})\end{array}$ & $\begin{array}{c}\text { Atrazine } \\
(\mu \mathrm{g} / \mathrm{L})\end{array}$ & $\begin{array}{c}\text { Benzo- } \\
\text { phenone } \\
(\mu \mathrm{g} / \mathrm{L})\end{array}$ & $\begin{array}{c}\text { beta- } \\
\text { Sitosterol } \\
(\mu \mathrm{g} / \mathrm{L})\end{array}$ & $\begin{array}{c}\text { beta- } \\
\text { Stigmastanol } \\
(\mu \mathrm{g} / \mathrm{L})\end{array}$ & $\begin{array}{c}\text { Bisphenol } \\
\text { A } \\
(\mu \mathrm{g} / \mathrm{L})\end{array}$ \\
\hline \multicolumn{16}{|c|}{ USGS-9D } \\
\hline 04-01-03 & 1100 & -- & -- & -- & -- & -- & -- & nd & -- & -- & nd & E0.1 & -- & -- & -- \\
\hline$*$ & 1105 & -- & -- & -- & -- & -- & -- & nd & -- & -- & nd & M & -- & -- & -- \\
\hline \multicolumn{16}{|c|}{ City of Columbia public-supply well 5} \\
\hline 04-03-03 & 0840 & -- & -- & -- & -- & -- & -- & nd & -- & -- & -- & E.1 & -- & -- & -- \\
\hline 08-28-03 & 1220 & -- & -- & -- & -- & -- & -- & nd & -- & -- & -- & $\mathrm{M}$ & -- & -- & -- \\
\hline \multicolumn{16}{|c|}{ Blew hole } \\
\hline 08-26-99 & 1340 & nd & nd & nd & nd & nd & nd & E0.043 & nd & nd & 0.192 & nd & nd & nd & nd \\
\hline $08-30-00$ & 0800 & nd & nd & nd & nd & nd & nd & E.059 & nd & nd & .073 & nd & nd & nd & nd \\
\hline $02-27-01$ & 1440 & nd & nd & nd & nd & nd & nd & E.007 & nd & nd & .013 & nd & nd & nd & nd \\
\hline \multicolumn{16}{|c|}{ City outflow } \\
\hline 08-31-99 & 1050 & nd & nd & nd & nd & nd & nd & -- & nd & nd & -- & nd & nd & nd & nd \\
\hline 08-29-00 & 1430 & nd & nd & nd & nd & nd & nd & -- & nd & nd & -- & nd & nd & nd & nd \\
\hline $02-28-01$ & 1440 & nd & nd & nd & nd & nd & nd & -- & nd & nd & -- & nd & nd & nd & nd \\
\hline 04-02-03 & 1400 & $\mathrm{M}$ & E1 & -- & E4 & M & E2 & nd & E0.3 & 0.6 & nd & .6 & -- & -- & 1 \\
\hline 04-09-03 & 1630 & E0.1 & E2 & M & E3 & M & E1 & nd & E. 2 & .8 & nd & .5 & E3 & E2 & M \\
\hline \multicolumn{16}{|c|}{ Eagle Bluffs outflow } \\
\hline 08-30-99 & 1540 & nd & nd & nd & nd & nd & nd & -- & nd & nd & -- & nd & nd & nd & nd \\
\hline 08-29-00 & 1530 & nd & nd & nd & nd & nd & nd & -- & nd & nd & -- & nd & nd & nd & nd \\
\hline 02-28-01 & 1300 & nd & nd & nd & nd & nd & nd & -- & nd & nd & -- & nd & nd & nd & nd \\
\hline \multicolumn{16}{|c|}{ Perche Creek } \\
\hline 08-26-99 & 0830 & nd & nd & nd & nd & nd & nd & E.029 & nd & nd & .154 & nd & nd & nd & nd \\
\hline 08-30-00 & 0930 & nd & nd & nd & nd & nd & nd & E.011 & nd & nd & .112 & nd & nd & nd & nd \\
\hline $02-27-01$ & 1450 & nd & nd & nd & nd & nd & nd & E.010 & nd & nd & .017 & nd & nd & nd & nd \\
\hline \multicolumn{16}{|c|}{ Missouri River } \\
\hline 04-02-03 & 1040 & -- & -- & -- & -- & -- & -- & nd & -- & $\mathrm{M}$ & nd & M & -- & -- & -- \\
\hline $08-27-03$ & 1540 & -- & -- & -- & -- & -- & -- & nd & E.1 & -- & nd & M & -- & -- & -- \\
\hline
\end{tabular}


Table 6. Concentrations of wastewater organic compounds and pesticides in samples from selected monitoring wells and surface-water sampling sites, 1999-2003.-Continued [ $\mu \mathrm{g} / \mathrm{L}$, micrograms per liter; constituent concentrations are dissolved, unless otherwise noted; CIAT, 2-chloro-4-isopropylamino-6-amino-s-triazine; AHTN, acetyl hexamethyl tetrahydronaphthalene, --, concentration less than reporting limit; nd, not determined; E, estimated; M, presence verified, but not quantified; *, replicate sample; DEET, n,n-diethyl-meta-toluamide]

\begin{tabular}{|c|c|c|c|c|c|c|c|c|c|c|c|c|c|c|}
\hline Date & $\begin{array}{c}\text { Caffeine } \\
(\mu \mathrm{g} / \mathrm{L})\end{array}$ & $\begin{array}{c}\text { Camphor } \\
(\mu \mathrm{g} / \mathrm{L})\end{array}$ & $\begin{array}{l}\text { Carbaryl } \\
(\mu \mathrm{g} / \mathrm{L})\end{array}$ & $\begin{array}{c}\text { Cholesterol } \\
(\mu \mathrm{g} / \mathrm{L})\end{array}$ & $\begin{array}{c}\text { Cotinine } \\
(\mu \mathrm{g} / \mathrm{L})\end{array}$ & $\begin{array}{c}\text { DEET } \\
(\mu \mathrm{g} / \mathrm{L})\end{array}$ & $\begin{array}{c}\text { Diazinon } \\
(\mu \mathrm{g} / \mathrm{L})\end{array}$ & $\begin{array}{c}\text { Diethoxy- } \\
\text { nonyl- } \\
\text { phenol } \\
(\mu \mathrm{g} / \mathrm{L})\end{array}$ & $\begin{array}{c}\text { Diethoxy- } \\
\text { octyl- } \\
\text { phenol } \\
(\mu \mathrm{g} / \mathrm{L})\end{array}$ & $\begin{array}{c}\text { Ethoxy- } \\
\text { octyl- } \\
\text { phenol } \\
(\mu \mathrm{g} / \mathrm{L})\end{array}$ & $\begin{array}{l}\text { HHCB } \\
(\mu \mathrm{g} / \mathrm{L})\end{array}$ & $\begin{array}{l}\text { Indole } \\
(\mu \mathrm{g} / \mathrm{L})\end{array}$ & $\begin{array}{l}\text { Isophorone } \\
(\mu \mathrm{g} / \mathrm{L})\end{array}$ & $\begin{array}{c}\text { Menthol } \\
(\mu \mathrm{g} / \mathrm{L})\end{array}$ \\
\hline \multicolumn{15}{|c|}{ USGS-9D } \\
\hline 04-01-03 & -- & -- & -- & -- & -- & E0.1 & -- & -- & -- & -- & -- & -- & -- & -- \\
\hline$*$ & -- & -- & -- & -- & -- & $\mathrm{M}$ & -- & -- & -- & -- & -- & -- & -- & -- \\
\hline \multicolumn{15}{|c|}{ City of Columbia public-supply well 5} \\
\hline 04-03-03 & -- & -- & -- & -- & -- & M & -- & -- & -- & -- & -- & -- & -- & -- \\
\hline $08-28-03$ & -- & -- & -- & -- & -- & M & -- & -- & -- & -- & -- & -- & -- & -- \\
\hline \multicolumn{15}{|c|}{ Blew hole } \\
\hline 08-26-99 & nd & nd & nd & nd & nd & nd & -- & nd & nd & nd & nd & nd & nd & nd \\
\hline $08-30-00$ & nd & nd & nd & nd & nd & nd & -- & nd & nd & nd & nd & nd & nd & nd \\
\hline $02-27-01$ & nd & nd & nd & nd & nd & nd & -- & nd & nd & nd & nd & nd & nd & nd \\
\hline \multicolumn{15}{|c|}{ City outflow } \\
\hline 08-31-99 & nd & nd & E0.053 & nd & nd & nd & 0.042 & nd & nd & nd & nd & nd & nd & nd \\
\hline $08-29-00$ & nd & nd & E.015 & nd & nd & nd & .058 & nd & nd & nd & nd & nd & nd & nd \\
\hline 02-28-01 & nd & nd & -- & nd & nd & nd & .028 & nd & nd & nd & nd & nd & nd & nd \\
\hline $04-02-03$ & E. 3 & -- & nd & E2 & E0.240 & .8 & -- & $\mathrm{E} 4$ & M & M & E0.1 & -- & E0.1 & -- \\
\hline 04-09-03 & 2.6 & M & nd & E3 & E. 420 & .5 & E.100 & E9 & M & E2 & E. 2 & E0.1 & -- & 0.9 \\
\hline \multicolumn{15}{|c|}{ Eagle Bluffs outflow } \\
\hline 08-30-99 & nd & nd & -- & nd & nd & nd & .028 & nd & nd & nd & nd & nd & nd & nd \\
\hline 08-29-00 & nd & nd & E.008 & nd & nd & nd & .047 & nd & nd & nd & nd & nd & nd & nd \\
\hline $02-28-01$ & nd & nd & -- & nd & nd & nd & .019 & nd & nd & nd & nd & nd & nd & nd \\
\hline \multicolumn{15}{|c|}{ Perche Creek } \\
\hline 08-26-99 & nd & nd & -- & nd & nd & nd & -- & nd & nd & nd & nd & nd & nd & nd \\
\hline 08-30-00 & nd & nd & -- & nd & nd & nd & .012 & nd & nd & nd & nd & nd & nd & nd \\
\hline $02-27-01$ & nd & nd & -- & nd & nd & nd & -- & nd & nd & nd & nd & nd & nd & nd \\
\hline \multicolumn{15}{|c|}{ Missouri River } \\
\hline $04-02-03$ & E.1 & -- & -- & -- & -- & M & -- & -- & -- & -- & -- & -- & -- & -- \\
\hline 08-27-03 & E.1 & -- & -- & -- & -- & E.1 & -- & -- & -- & -- & -- & -- & -- & -- \\
\hline
\end{tabular}


[ $\mu \mathrm{g} / \mathrm{L}$, micrograms per liter; constituent concentrations are dissolved, unless otherwise noted; CIAT, 2-chloro-4-isopropylamino-6-amino-s-triazine; AHTN, acetyl hexamethyl tetrahydronaphthalene; --, concentration less than reporting limit; nd, not determined; E, estimated; M, presence verified, but not quantified; *, replicate sample; DEET, n,n-diethyl-meta-toluamide]

\begin{tabular}{|c|c|c|c|c|c|c|c|c|c|c|c|c|c|c|c|}
\hline Date & $\begin{array}{c}\text { Methyl } \\
\text { salicylate } \\
(\mu \mathrm{g} / \mathrm{L})\end{array}$ & $\begin{array}{c}\text { Metolachlor } \\
(\mu \mathrm{g} / \mathrm{L})\end{array}$ & $\begin{array}{c}\text { Metribuzin } \\
(\mu \mathrm{g} / \mathrm{L})\end{array}$ & $\begin{array}{c}\text { p-Cresol } \\
(\mu \mathrm{g} / \mathrm{L})\end{array}$ & $\begin{array}{l}\text { Phenol } \\
(\mu \mathrm{g} / \mathrm{L})\end{array}$ & $\begin{array}{c}\text { Prometon } \\
(\mu \mathrm{g} / \mathrm{L})\end{array}$ & $\begin{array}{c}\text { Simazine } \\
(\mu \mathrm{g} / \mathrm{L})\end{array}$ & $\begin{array}{c}\text { Tribromo- } \\
\text { methane } \\
(\mu \mathrm{g} / \mathrm{L})\end{array}$ & $\begin{array}{c}\text { Tributyl } \\
\text { phosphate } \\
(\mu \mathrm{g} / \mathrm{L})\end{array}$ & $\begin{array}{c}\text { Triclosan } \\
(\mu \mathrm{g} / \mathrm{L})\end{array}$ & $\begin{array}{l}\text { Triethyl } \\
\text { citrate } \\
(\mu \mathrm{g} / \mathrm{L})\end{array}$ & $\begin{array}{c}\text { Triphenyl } \\
\text { phosphate } \\
(\mu \mathrm{g} / \mathrm{L})\end{array}$ & $\begin{array}{c}\text { Tris } \\
\text { (2-butoxy- } \\
\text { ethyl) } \\
\text { phosphate } \\
(\mu \mathrm{g} / \mathrm{L})\end{array}$ & $\begin{array}{c}\text { Tris } \\
\text { (2-chloro- } \\
\text { ethyl) } \\
\text { phosphate } \\
(\mu \mathrm{g} / \mathrm{L})\end{array}$ & $\begin{array}{c}\text { Tris } \\
\text { (dichloro- } \\
\text { i-Pr) } \\
\text { phosphate } \\
(\mu \mathrm{g} / \mathrm{L})\end{array}$ \\
\hline \multicolumn{16}{|c|}{ USGS-9D } \\
\hline \multirow[t]{2}{*}{ 04-01-03 } & E0.2 & -- & nd & -- & E0.2 & -- & nd & -- & -- & -- & -- & -- & -- & -- & -- \\
\hline & E.1 & -- & nd & -- & .6 & -- & nd & -- & -- & -- & -- & -- & -- & -- & -- \\
\hline \multicolumn{16}{|c|}{ City of Columbia public-supply well 5} \\
\hline 04-03-03 & M & -- & nd & -- & E.2 & -- & nd & -- & -- & -- & -- & -- & -- & -- & -- \\
\hline $08-28-03$ & -- & -- & nd & -- & -- & -- & nd & -- & -- & -- & -- & -- & -- & -- & -- \\
\hline \multicolumn{16}{|c|}{ Blew hole } \\
\hline 08-26-99 & nd & 0.064 & -- & nd & nd & -- & -- & nd & nd & nd & nd & nd & nd & nd & nd \\
\hline $08-30-00$ & nd & -- & -- & nd & nd & E0.01 & -- & nd & nd & nd & nd & nd & nd & nd & nd \\
\hline $02-27-01$ & nd & E.004 & 0.031 & nd & nd & -- & -- & nd & nd & nd & nd & nd & nd & nd & nd \\
\hline \multicolumn{16}{|c|}{ City outflow } \\
\hline 08-31-99 & nd & -- & -- & nd & nd & -- & -- & nd & nd & nd & nd & nd & nd & nd & nd \\
\hline 08-29-00 & nd & -- & -- & nd & nd & -- & -- & nd & nd & nd & nd & nd & nd & nd & nd \\
\hline $02-28-01$ & nd & -- & -- & nd & nd & -- & -- & nd & nd & nd & nd & nd & nd & nd & nd \\
\hline $04-02-03$ & E.1 & -- & nd & M & E. 2 & -- & nd & -- & E0.3 & M & E0.2 & E0.1 & 2.0 & E0.3 & E0.2 \\
\hline 04-09-03 & -- & -- & nd & M & E. 5 & 1.7 & nd & M & E. 3 & M & E. 3 & E. 1 & E2.6 & E. 3 & E. 3 \\
\hline \multicolumn{16}{|c|}{ Eagle Bluffs outflow } \\
\hline 08-30-99 & nd & -- & -- & nd & nd & -- & -- & nd & nd & nd & nd & nd & nd & nd & nd \\
\hline $08-29-00$ & nd & -- & -- & nd & nd & E.02 & -- & nd & nd & nd & nd & nd & nd & nd & nd \\
\hline 02-28-01 & nd & -- & -- & nd & nd & -- & -- & nd & nd & nd & nd & nd & nd & nd & nd \\
\hline \multicolumn{16}{|c|}{ Perche Creek } \\
\hline 08-26-99 & nd & .020 & -- & nd & nd & -- & -- & nd & nd & nd & nd & nd & nd & nd & nd \\
\hline 08-30-00 & nd & .013 & -- & nd & nd & E.01 & 0.008 & nd & nd & nd & nd & nd & nd & nd & nd \\
\hline $02-27-01$ & nd & E.005 & .026 & nd & nd & -- & -- & nd & nd & nd & nd & nd & nd & nd & nd \\
\hline \multicolumn{16}{|c|}{ Missouri River } \\
\hline 04-02-03 & M & M & nd & -- & E. 2 & -- & nd & M & -- & -- & -- & E. 1 & E. 3 & -- & -- \\
\hline $08-27-03$ & -- & M & nd & -- & .6 & -- & nd & -- & -- & -- & -- & -- & -- & -- & -- \\
\hline
\end{tabular}


Table 7. Altitude of water levels in monitoring wells, 2004-2005.

[Altitudes are in feet above National Geodetic Vertical Datum of 1929 (NGVD 29); ft, feet; --, no data]

\begin{tabular}{|c|c|c|c|c|c|}
\hline $\begin{array}{c}\text { Site } \\
\text { (fig. 3) }\end{array}$ & Date & $\begin{array}{c}\text { Altitude } \\
\text { (ft) }\end{array}$ & $\begin{array}{c}\text { Site } \\
\text { (fig. 3) }\end{array}$ & Date & $\begin{array}{c}\text { Altitude } \\
\text { (ft) }\end{array}$ \\
\hline \multirow[t]{4}{*}{ MW-101 } & 02-24-2004 & 567.35 & MW-111 & $02-24-2004$ & 551.84 \\
\hline & $08-31-2004$ & 559.56 & & $08-31-2004$ & 557.65 \\
\hline & 03-08-2005 & 560.81 & & 03-08-2005 & 553.87 \\
\hline & 08-02-2005 & 557.11 & & 08-02-2005 & 552.77 \\
\hline \multirow[t]{4}{*}{ MW-102 } & 02-24-2004 & 558.24 & MW-112 & 02-24-2004 & 553.33 \\
\hline & 08-31-2004 & 561.16 & & 08-31-2004 & 558.12 \\
\hline & 03-08-2005 & 563.20 & & 03-08-2005 & 556.45 \\
\hline & 08-02-2005 & 557.96 & & 08-02-2005 & 555.08 \\
\hline \multirow[t]{4}{*}{ MW-103 } & 02-24-2004 & 556.57 & MW-113 & 02-24-2004 & 553.40 \\
\hline & 08-31-2004 & 560.41 & & $08-31-2004$ & 562.17 \\
\hline & 03-08-2005 & 561.28 & & 03-08-2005 & 554.60 \\
\hline & 08-02-2005 & 556.98 & & $08-02-2005$ & 554.51 \\
\hline \multirow[t]{4}{*}{ MW-104 } & 02-24-2004 & 554.65 & MW-114 & 02-24-2004 & 556.31 \\
\hline & $08-31-2004$ & 562.07 & & 08-31-2004 & 562.81 \\
\hline & 03-08-2005 & 557.27 & & 03-08-2005 & 559.63 \\
\hline & 08-02-2005 & 555.70 & & 08-02-2005 & 556.71 \\
\hline \multirow[t]{4}{*}{ MW-105 } & 02-24-2004 & 554.33 & MW-115 & 02-24-2004 & 552.04 \\
\hline & 08-31-2004 & 559.34 & & 08-31-2004 & 557.78 \\
\hline & 03-08-2005 & 556.84 & & 03-08-2005 & 553.61 \\
\hline & 08-02-2005 & 555.62 & & 08-02-2005 & 553.47 \\
\hline \multirow[t]{4}{*}{ MW-106 } & 02-24-2004 & 554.72 & MW-116 & 02-24-2004 & 552.75 \\
\hline & $08-31-2004$ & 559.66 & & $08-31-2004$ & 557.29 \\
\hline & 03-08-2005 & 558.18 & & 03-08-2005 & 555.72 \\
\hline & 08-02-2005 & 556.21 & & 08-02-2005 & 554.50 \\
\hline \multirow[t]{4}{*}{ MW-107 } & 02-24-2004 & 554.83 & MW-117 & 02-24-2004 & 551.88 \\
\hline & $08-31-2004$ & 560.02 & & $08-31-2004$ & 557.73 \\
\hline & 03-08-2005 & 558.45 & & 03-08-2005 & 553.70 \\
\hline & 08-02-2005 & 556.43 & & 08-02-2005 & 552.72 \\
\hline \multirow[t]{4}{*}{ MW-108 } & 02-24-2004 & 556.01 & MW-118 & 02-24-2004 & 551.89 \\
\hline & 08-31-2004 & 560.70 & & $08-31-2004$ & 559.37 \\
\hline & 03-08-2005 & 560.50 & & 03-08-2005 & 554.58 \\
\hline & 08-02-2005 & 557.26 & & 08-02-2005 & 553.95 \\
\hline \multirow[t]{4}{*}{ MW-109 } & 02-24-2004 & 554.62 & MW-117 & 02-24-2004 & 551.88 \\
\hline & 08-31-2004 & 562.44 & & $08-31-2004$ & 557.73 \\
\hline & 03-08-2005 & 557.14 & & 03-08-2005 & 553.70 \\
\hline & 08-02-2005 & 555.69 & & 08-02-2005 & 552.72 \\
\hline \multirow[t]{4}{*}{ MW-110 } & 02-24-2004 & 553.70 & MW-118 & 02-24-2004 & 551.89 \\
\hline & $08-31-2004$ & 561.10 & & 08-31-2004 & 559.37 \\
\hline & 03-08-2005 & 555.94 & & 03-08-2005 & 554.58 \\
\hline & 08-02-2005 & 555.11 & & 08-02-2005 & 553.95 \\
\hline
\end{tabular}


Table 7. Altitude of water levels in monitoring wells, 2004-2005.-Continued

[Altitudes are in feet above National Geodetic Vertical Datum of 1929 (NGVD 29); ft, feet; --, no data]

\begin{tabular}{|c|c|c|c|c|c|}
\hline $\begin{array}{c}\text { Site } \\
\text { (fig. 3) }\end{array}$ & Date & $\begin{array}{c}\text { Altitude } \\
\text { (ft) }\end{array}$ & $\begin{array}{c}\text { Site } \\
\text { (fig. 3) }\end{array}$ & Date & $\begin{array}{c}\text { Altitude } \\
\text { (ft) }\end{array}$ \\
\hline \multirow[t]{4}{*}{ MW-119 } & $02-24-2004$ & 551.21 & MW-129 & 02-24-2004 & 551.19 \\
\hline & 08-31-2004 & 556.42 & & 08-31-2004 & 555.34 \\
\hline & 03-08-2005 & 554.44 & & 03-08-2005 & 554.47 \\
\hline & 08-02-2005 & 553.00 & & 08-02-2005 & 553.52 \\
\hline \multirow[t]{4}{*}{ MW-120 } & 02-24-2004 & 548.90 & MW-130 & 02-24-2004 & 552.78 \\
\hline & 08-31-2004 & 554.17 & & 08-31-2004 & 556.17 \\
\hline & 03-08-2005 & 554.50 & & 03-08-2005 & 555.69 \\
\hline & 08-02-2005 & 551.78 & & 08-02-2005 & 554.33 \\
\hline \multirow[t]{4}{*}{ MW-121 } & 02-24-2004 & 550.83 & MW-131 & 02-24-2004 & 553.71 \\
\hline & 08-31-2004 & 555.40 & & 08-31-2004 & -- \\
\hline & 03-08-2005 & 553.94 & & 03-08-2005 & 556.02 \\
\hline & 08-02-2005 & 552.77 & & 08-02-2005 & 555.74 \\
\hline \multirow[t]{4}{*}{ MW-122 } & 02-24-2004 & 549.57 & MW-132 & 02-24-2004 & 551.15 \\
\hline & 08-31-2004 & 553.99 & & 08-31-2004 & 555.42 \\
\hline & 03-08-2005 & 554.05 & & 03-08-2005 & 554.48 \\
\hline & 08-02-2005 & 551.95 & & 08-02-2005 & 553.66 \\
\hline \multirow[t]{4}{*}{ MW-123 } & 02-24-2004 & 548.68 & MW-133 & 02-24-2004 & 549.86 \\
\hline & $08-31-2004$ & 553.67 & & 08-31-2004 & 553.74 \\
\hline & 03-08-2005 & 553.74 & & 03-08-2005 & 553.39 \\
\hline & 08-02-2005 & 551.13 & & 08-02-2005 & 552.71 \\
\hline \multirow[t]{4}{*}{ MW-124 } & 02-24-2004 & 548.81 & MW-134 & 02-24-2004 & 549.20 \\
\hline & 08-31-2004 & 553.95 & & 08-31-2004 & 554.11 \\
\hline & 03-08-2005 & 552.96 & & 03-08-2005 & 553.47 \\
\hline & 08-02-2005 & 551.77 & & 08-02-2005 & 552.37 \\
\hline \multirow[t]{4}{*}{ MW-125 } & 02-24-2004 & 549.00 & MW-135 & 02-24-2004 & 551.57 \\
\hline & 08-31-2004 & 555.69 & & 08-31-2004 & 561.54 \\
\hline & 03-08-2005 & 552.99 & & 03-08-2005 & 554.89 \\
\hline & 08-02-2005 & 551.79 & & $08-02-2005$ & 554.63 \\
\hline \multirow[t]{4}{*}{ MW-126 } & 02-24-2004 & 549.91 & MW-136 & 02-24-2004 & 550.02 \\
\hline & 08-31-2004 & 554.18 & & 08-31-2004 & 557.68 \\
\hline & 03-08-2005 & 553.55 & & 03-08-2005 & 554.62 \\
\hline & 08-02-2005 & 552.53 & & 08-02-2005 & 553.09 \\
\hline \multirow[t]{4}{*}{ MW-127 } & 02-24-2004 & 551.87 & MW-137 & 02-24-2004 & 552.39 \\
\hline & 08-31-2004 & 555.58 & & 08-31-2004 & 562.25 \\
\hline & 03-08-2005 & 554.31 & & 03-08-2005 & 555.32 \\
\hline & 08-02-2005 & 553.26 & & 08-02-2005 & 555.03 \\
\hline \multirow[t]{4}{*}{ MW-128 } & 02-24-2004 & 552.30 & MW-138 & 02-24-2004 & 549.15 \\
\hline & 08-31-2004 & 556.28 & & 08-31-2004 & 556.39 \\
\hline & 03-08-2005 & 554.89 & & 03-08-2005 & 553.64 \\
\hline & 08-02-2005 & -- & & 08-02-2005 & 551.56 \\
\hline
\end{tabular}


Table 7. Altitude of water levels in monitoring wells, 2004-2005.-Continued

[Altitudes are in feet above National Geodetic Vertical Datum of 1929 (NGVD 29); ft, feet; --, no data]

\begin{tabular}{|c|c|c|c|c|c|}
\hline $\begin{array}{c}\text { Site } \\
\text { (fig. 3) }\end{array}$ & Date & $\begin{array}{c}\text { Altitude } \\
\text { (ft) }\end{array}$ & $\begin{array}{c}\text { Site } \\
\text { (fig. 3) }\end{array}$ & Date & $\begin{array}{c}\text { Altitude } \\
\text { (ft) }\end{array}$ \\
\hline \multirow[t]{4}{*}{ MW-139 } & $02-24-2004$ & 550.04 & MW-150 & $02-24-2004$ & 566.71 \\
\hline & 08-31-2004 & 554.53 & & 08-31-2004 & 566.69 \\
\hline & 03-08-2005 & 553.96 & & 03-08-2005 & 566.58 \\
\hline & 08-02-2005 & 553.27 & & 08-02-2005 & -- \\
\hline \multirow[t]{4}{*}{ MW-140 } & 02-24-2004 & 560.95 & MW-151 & 02-24-2004 & 552.64 \\
\hline & 08-31-2004 & 562.56 & & 08-31-2004 & 557.86 \\
\hline & 03-08-2005 & 559.09 & & 03-08-2005 & 556.22 \\
\hline & 08-02-2005 & 557.68 & & 08-02-2005 & 557.27 \\
\hline \multirow[t]{4}{*}{ MW-141 } & 02-24-2004 & 552.53 & MW-152 & 02-24-2004 & 555.90 \\
\hline & 08-31-2004 & 556.17 & & 08-31-2004 & 566.87 \\
\hline & 03-08-2005 & 555.68 & & 03-08-2005 & 557.10 \\
\hline & 08-02-2005 & 554.76 & & 08-02-2005 & 558.35 \\
\hline \multirow[t]{4}{*}{ MW-142 } & 02-24-2004 & 576.20 & MW-153 & 02-24-2004 & 555.82 \\
\hline & 08-31-2004 & 575.45 & & 08-31-2004 & 565.96 \\
\hline & 03-08-2005 & 576.55 & & 03-08-2005 & 557.99 \\
\hline & 08-02-2005 & 574.36 & & 08-02-2005 & 559.02 \\
\hline \multirow[t]{4}{*}{ MW-143 } & 02-24-2004 & 568.29 & MW-154 & 02-24-2004 & 551.24 \\
\hline & 08-31-2004 & 568.38 & & 08-31-2004 & 555.39 \\
\hline & 03-08-2005 & 569.10 & & 03-08-2005 & 554.76 \\
\hline & 08-02-2005 & 567.16 & & 08-02-2005 & 554.26 \\
\hline \multirow[t]{4}{*}{ MW-144 } & 02-24-2004 & 550.25 & MW-155 & 02-24-2004 & 551.49 \\
\hline & 08-31-2004 & 555.90 & & 08-31-2004 & 555.60 \\
\hline & 03-08-2005 & 554.10 & & 03-08-2005 & 556.43 \\
\hline & 08-02-2005 & 553.65 & & 08-02-2005 & 554.74 \\
\hline \multirow[t]{4}{*}{ MW-145 } & 02-24-2004 & 553.33 & MW1-1A & 02-24-2004 & 569.01 \\
\hline & $08-31-2004$ & 562.62 & & 08-31-2004 & 569.21 \\
\hline & 03-08-2005 & 555.94 & & 03-08-2005 & 570.06 \\
\hline & 08-02-2005 & 556.11 & & 08-02-2005 & 568.37 \\
\hline \multirow[t]{4}{*}{ MW-146 } & 02-24-2004 & 552.64 & MW1-1B & 02-24-2004 & 562.15 \\
\hline & $08-31-2004$ & 560.90 & & 08-31-2004 & 563.30 \\
\hline & 03-08-2005 & 555.51 & & 03-08-2005 & 563.55 \\
\hline & 08-02-2005 & 555.85 & & 08-02-2005 & 561.37 \\
\hline \multirow[t]{4}{*}{ MW-147 } & 02-24-2004 & 548.54 & MW1-2A & 02-24-2004 & 552.68 \\
\hline & 08-31-2004 & 554.50 & & 08-31-2004 & 556.48 \\
\hline & 03-08-2005 & 552.81 & & 03-08-2005 & 555.61 \\
\hline & 08-02-2005 & -- & & 08-02-2005 & 554.72 \\
\hline \multirow[t]{4}{*}{ MW-148 } & 02-24-2004 & 555.12 & MW1-2B & 02-24-2004 & 552.68 \\
\hline & 08-31-2004 & 561.02 & & 08-31-2004 & 556.47 \\
\hline & 03-08-2005 & 558.42 & & 03-08-2005 & 555.61 \\
\hline & 08-02-2005 & 556.08 & & 08-02-2005 & 554.74 \\
\hline
\end{tabular}


Table 7. Altitude of water levels in monitoring wells, 2004-2005.-Continued

[Altitudes are in feet above National Geodetic Vertical Datum of 1929 (NGVD 29); ft, feet; --, no data]

\begin{tabular}{|c|c|c|c|c|c|}
\hline $\begin{array}{c}\text { Site } \\
\text { (fig. 3) }\end{array}$ & Date & $\begin{array}{c}\text { Altitude } \\
\text { (ft) }\end{array}$ & $\begin{array}{c}\text { Site } \\
\text { (fig. 3) }\end{array}$ & Date & $\begin{array}{c}\text { Altitude } \\
\text { (ft) }\end{array}$ \\
\hline \multirow[t]{4}{*}{ MW1-3A } & $02-24-2004$ & 551.95 & MW4-2A & 02-24-2004 & 555.48 \\
\hline & 08-31-2004 & 555.82 & & 08-31-2004 & 562.15 \\
\hline & 03-08-2005 & 555.15 & & 03-08-2005 & 558.89 \\
\hline & 08-02-2005 & 554.38 & & 08-02-2005 & 556.73 \\
\hline \multirow[t]{4}{*}{ MW1-3B } & 02-24-2004 & 551.81 & MW4-2B & 02-24-2004 & 555.50 \\
\hline & 08-31-2004 & 555.72 & & 08-31-2004 & 562.17 \\
\hline & 03-08-2005 & 555.04 & & 03-08-2005 & 558.86 \\
\hline & 08-02-2005 & 554.26 & & 08-02-2005 & 556.71 \\
\hline \multirow[t]{4}{*}{ MW1-4A } & 02-24-2004 & 572.11 & USGS-1 & 02-24-2004 & 553.61 \\
\hline & 08-31-2004 & 571.66 & & 08-31-2004 & 559.05 \\
\hline & 03-08-2005 & 572.62 & & 03-08-2005 & 556.04 \\
\hline & 08-02-2005 & 570.86 & & 08-02-2005 & 555.22 \\
\hline \multirow[t]{4}{*}{ MW1-4B } & 02-24-2004 & 561.68 & USGS-2S & 02-24-2004 & 556.74 \\
\hline & 08-31-2004 & 562.85 & & 08-31-2004 & 561.34 \\
\hline & 03-08-2005 & 562.91 & & 03-08-2005 & 561.47 \\
\hline & 08-02-2005 & 560.44 & & 08-02-2005 & 557.53 \\
\hline \multirow[t]{4}{*}{ MW2-1A } & 02-24-2004 & 552.13 & USGS-2D & 02-24-2004 & 556.76 \\
\hline & 08-31-2004 & 556.85 & & 08-31-2004 & 561.38 \\
\hline & 03-08-2005 & 554.13 & & 03-08-2005 & 561.37 \\
\hline & 08-02-2005 & 553.51 & & 08-02-2005 & 557.53 \\
\hline \multirow[t]{4}{*}{ MW2-1B } & 02-24-2004 & -- & USGS-3S & 02-24-2004 & 556.08 \\
\hline & $08-31-2004$ & -- & & 08-31-2004 & 560.62 \\
\hline & 03-08-2005 & -- & & 03-08-2005 & 559.25 \\
\hline & 08-02-2005 & -- & & 08-02-2005 & 556.29 \\
\hline \multirow[t]{4}{*}{ MW3-1A } & 02-24-2004 & 553.02 & USGS-3D & 02-24-2004 & 555.85 \\
\hline & 08-31-2004 & 557.93 & & 08-31-2004 & 560.40 \\
\hline & 03-08-2005 & 555.56 & & 03-08-2005 & 558.99 \\
\hline & 08-02-2005 & 554.71 & & 08-02-2005 & 556.07 \\
\hline \multirow[t]{4}{*}{ MW3-1B } & 02-24-2004 & 552.96 & USGS-4 & 02-24-2004 & 555.66 \\
\hline & 08-31-2004 & 557.90 & & 08-31-2004 & 561.88 \\
\hline & 03-08-2005 & 555.48 & & 03-08-2005 & 559.56 \\
\hline & 08-02-2005 & 554.64 & & 08-02-2005 & 556.58 \\
\hline \multirow[t]{4}{*}{ MW4-1A } & 02-24-2004 & 554.19 & USGS-5S & 02-24-2004 & 559.26 \\
\hline & 08-31-2004 & 559.79 & & 08-31-2004 & 560.07 \\
\hline & 03-08-2005 & 557.44 & & 03-08-2005 & 563.89 \\
\hline & 08-02-2005 & 555.86 & & 08-02-2005 & 558.48 \\
\hline \multirow[t]{4}{*}{ MW4-1B } & 02-24-2004 & 554.16 & USGS-5D & 02-24-2004 & 559.24 \\
\hline & 08-31-2004 & 559.96 & & 08-31-2004 & 560.09 \\
\hline & 03-08-2005 & 557.26 & & 03-08-2005 & 563.89 \\
\hline & 08-02-2005 & 555.74 & & 08-02-2005 & 558.53 \\
\hline
\end{tabular}


Table 7. Altitude of water levels in monitoring wells, 2004-2005._-Continued

[Altitudes are in feet above National Geodetic Vertical Datum of 1929 (NGVD 29); ft, feet; --, no data]

\begin{tabular}{|c|c|c|c|c|c|}
\hline $\begin{array}{c}\text { Site } \\
\text { (fig. 3) }\end{array}$ & Date & $\begin{array}{c}\text { Altitude } \\
\text { (ft) }\end{array}$ & $\begin{array}{c}\text { Site } \\
\text { (fig. 3) }\end{array}$ & Date & $\begin{array}{c}\text { Altitude } \\
\text { (ft) }\end{array}$ \\
\hline \multirow[t]{4}{*}{ USGS-6 } & $02-24-2004$ & 554.65 & USGS-9D & $02-24-2004$ & -- \\
\hline & 08-31-2004 & 560.70 & & $08-31-2004$ & -- \\
\hline & 03-08-2005 & 556.07 & & 03-08-2005 & -- \\
\hline & 08-02-2005 & 555.18 & & 08-02-2005 & -- \\
\hline \multirow[t]{4}{*}{ USGS-7 } & 02-24-2004 & 553.22 & SP11 & 02-24-2004 & 559.33 \\
\hline & 08-31-2004 & 561.70 & & $08-31-2004$ & 560.05 \\
\hline & 03-08-2005 & 555.24 & & 03-08-2005 & 563.81 \\
\hline & 08-02-2005 & 554.80 & & 08-02-2005 & 560.76 \\
\hline \multirow[t]{4}{*}{ USGS-8S } & 02-24-2004 & 549.80 & SP4 & 02-24-2004 & 563.77 \\
\hline & 08-31-2004 & 553.29 & & $08-31-2004$ & -- \\
\hline & 03-08-2005 & 554.57 & & 03-08-2005 & 563.75 \\
\hline & 08-02-2005 & 551.36 & & 08-02-2005 & 563.22 \\
\hline \multirow[t]{4}{*}{ USGS-8D } & 02-24-2004 & 549.60 & MW13-67 & $02-24-2004$ & 549.49 \\
\hline & 08-31-2004 & 552.59 & & 08-31-2004 & 551.72 \\
\hline & 03-08-2005 & 554.48 & & 03-08-2005 & 554.42 \\
\hline & 08-02-2005 & 550.56 & & 08-02-2005 & 549.74 \\
\hline \multirow[t]{4}{*}{ USGS-9S } & 02-24-2004 & 554.03 & MW28-67 & 02-24-2004 & 557.60 \\
\hline & 08-31-2004 & 560.40 & & 08-31-2004 & 560.76 \\
\hline & 03-08-2005 & 558.21 & & 03-08-2005 & 561.65 \\
\hline & 08-02-2005 & 559.07 & & 08-02-2005 & 558.15 \\
\hline
\end{tabular}




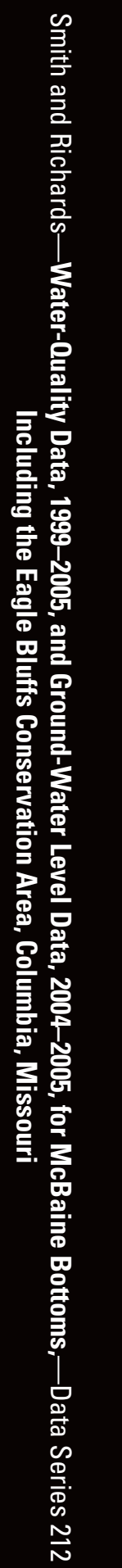

